Supporting Information for:

\title{
Pyrones Identified as LuxR Signal Molecules in Photorhabdus and Their Synthetic Analogues Can Alter Multicellular Phenotypic Behavior of Bacillus atropheaus
}

Aobha Hickey, ${ }^{a}$ Leticia M. Pardo, ${ }^{a}$ F. Jerry Reen*b,c and Gerard P. McGlacken*a,c

aSchool of Chemistry, Analytical and Biological Chemistry Research Facility, 'bchool of Microbiology, 'Synthesis and Solid State Pharmaceutical Centre, University College Cork, Ireland.

*g.mcglacken@ucc.ie,*j.reen@ucc.ie

\section{Contents}

Synthesis and Characterisation of 2-Pyrones and 2-Pyridones

Biological Figures and Tables

${ }^{1} \mathrm{H}$ and ${ }^{13} \mathrm{C}$ NMR Spectra 


\section{Synthesis and Characterisation of 2-Pyrones and 2-Pyridones}

\section{Synthesis of 3-alkyl-4-hydroxy-6-methyl-2H-pyran-2-ones}

Representative procedure for reductive alkylation at C3 of 2-pyrones

To a round bottom flask in open air was added 2- pyrone (1.0 equiv.), corresponding aldehyde (3.0 equiv.), diethyl 1,4-dihydro-2,6-dimethyl-3,5-pyridinedicarboxylate (1.2 equiv.) and DCM (15 mL/mmol). L-proline (20 mol\%) was then added and the sides of the flask were rinsed again with DCM. The resulting reaction mixture was allowed to stir vigorously for $16 \mathrm{~h}$ at r.t. The reaction mixture was then concentrated under reduced pressure. The crude product was purified by column chromatography (hexanes:EtOAc 9:1 to $7: 3) .^{1}$

\section{4-Hydroxy-6-methyl-3-propyl-2H-pyran-2-one (2) ${ }^{1}$}

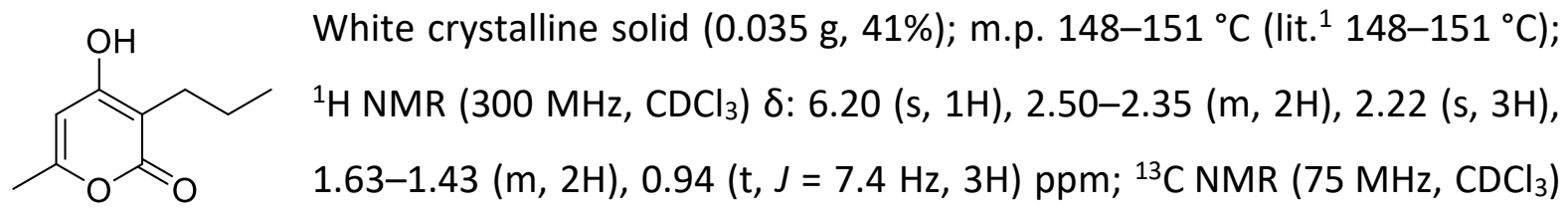

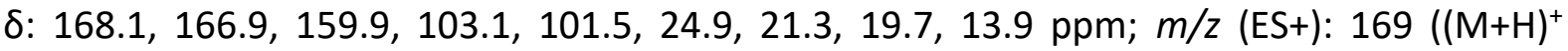
$100 \%)$.

\section{3-Butyl-4-hydroxy-6-methyl-2H-pyran-2-one (3)}

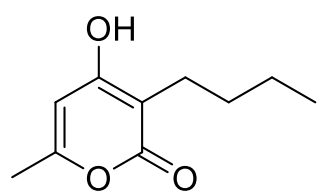

White crystalline solid $(0.045 \mathrm{~g}, 49 \%)$; m.p. $130-133^{\circ} \mathrm{C}$ (lit. $^{2} 132-$ $\left.133^{\circ} \mathrm{C}\right) ; \mathrm{IR}\left(\right.$ film) $V_{\max } 3583,1650,1575,1447,1292,1127 \mathrm{~cm}^{-1} ;{ }^{1} \mathrm{H} N M R$ (300 MHz, $\left.\mathrm{CDCl}_{3}\right) \delta: 9.73$ (bs, 1H), $6.18(\mathrm{~s}, 1 \mathrm{H}), 2.56-2.35(\mathrm{~m}, 2 \mathrm{H}), 2.22$ (s, 3H), 1.61-1.20 (m, 4H), $0.91(\mathrm{t}, J=7.2 \mathrm{~Hz}, 3 \mathrm{H}) \mathrm{ppm} ;{ }^{13} \mathrm{C} \mathrm{NMR}\left(75 \mathrm{MHz}, \mathrm{CDCl}_{3}\right) \delta: 168.5$, 167.5, 159.8, 103.4, 101.8, 30.2, 22.74, 22.66, 19.6, 14.0 ppm; HRMS (ESI-TOF) m/z: [M+H] ${ }^{+}$ calcd for $\mathrm{C}_{10} \mathrm{H}_{15} \mathrm{O}_{3}$ : 183.1016; found: 183.1010 .

\section{4-Hydroxy-6-methyl-3-pentyl-2H-pyran-2-one (4)}<smiles>CCCCCc1c(O)cc(C)oc1=O</smiles>
Off-white solid (0.179 g, 91\%); m.p. $132-136^{\circ} \mathrm{C}$; IR (film) $v_{\max } 3208$, 1648, 1573, 1447, 1274, $1128 \mathrm{~cm}^{-1} ;{ }^{1} \mathrm{H} \mathrm{NMR}\left(300 \mathrm{MHz}, \mathrm{CDCl}_{3}\right) \delta$ : 10.60 (bs, 1H), 6.27 (s, 1H), 2.55-2.35 (m, 2H), 2.22 (s, 3H), 1.59- 
$1.20(\mathrm{~m}, 6 \mathrm{H}), 0.87(\mathrm{t}, J=6.9 \mathrm{~Hz}, 3 \mathrm{H}) \mathrm{ppm} ;{ }^{13} \mathrm{C} \mathrm{NMR}\left(75 \mathrm{MHz}, \mathrm{CDCl}_{3}\right)$ \&: 168.6, 167.7, 159.8, 103.4, 101.9, 31.8, 27.8, 23.0, 22.6, 19.7, 14.1 ppm; HRMS (ESI-TOF) $\mathrm{m} / \mathrm{z}$ : [M+H] ${ }^{+}$calcd for $\mathrm{C}_{11} \mathrm{H}_{17} \mathrm{O}_{3}$ : 197.1211; found: 197.1208 .

\section{3-Hexyl-4-hydroxy-6-methyl-2H-pyran-2-one (5) ${ }^{1}$}

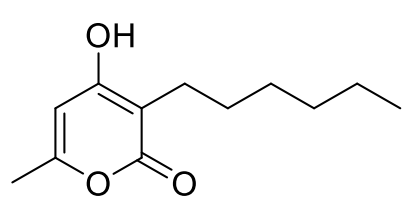

White solid (0.376 g, 90\%); m.p. $115-118^{\circ} \mathrm{C}$ (lit. ${ }^{1} 120-121^{\circ} \mathrm{C}$ );

${ }^{1} \mathrm{H}$ NMR $\left(300 \mathrm{MHz}, \mathrm{CDCl}_{3}\right.$ ) $\delta: 10.52$ (bs, $\left.1 \mathrm{H}\right), 6.27$ (s, 1H), 2.55-2.36 $(\mathrm{m}, 2 \mathrm{H}), 2.22(\mathrm{~s}, 3 \mathrm{H}), 1.61-1.18(\mathrm{~m}, 8 \mathrm{H}), 0.86(\mathrm{t}, J=6.6 \mathrm{~Hz}, 3 \mathrm{H})$ ppm; ${ }^{13} \mathrm{C}$ NMR $\left(75 \mathrm{MHz}, \mathrm{CDCl}_{3}\right.$ ) $\delta: 168.6,167.6,159.8,103.4,101.9,31.8,29.3,28.0,23.0$, 22.7, 19.6, 14.1 ppm; m/z (ES-): 209 ((M-H)- 100\%).

\section{3-Heptyl-4-hydroxy-6-methyl-2H-pyran-2-one (6) ${ }^{1}$}

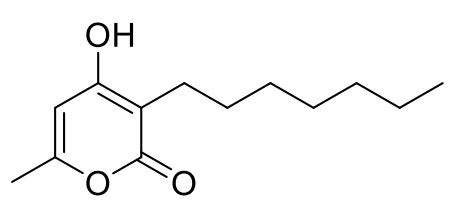

Off-white solid $(0.210 \mathrm{~g}, 93 \%) ;$ m.p. $119-124{ }^{\circ} \mathrm{C}$; ${ }^{1} \mathrm{H}$ NMR

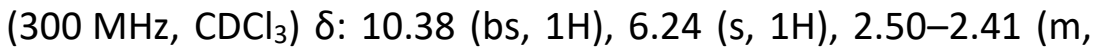
$2 \mathrm{H}), 2.22(\mathrm{~s}, 3 \mathrm{H}), 1.60-1.14(\mathrm{~m}, 10 \mathrm{H}), 0.86(\mathrm{t}, J=6.7 \mathrm{~Hz}, 3 \mathrm{H})$ ppm; ${ }^{13} \mathrm{C} \mathrm{NMR}\left(75 \mathrm{MHz}, \mathrm{CDCl}_{3}\right.$ ) $\delta: 168.5,167.6,159.7,103.9,101.8,31.9,29.6,29.3,28.1$, 23.0, 22.7, 19.6, $14.1 \mathrm{ppm} ; \mathrm{m} / \mathrm{z}(\mathrm{ES}+): 225\left((\mathrm{M}+\mathrm{H})^{+}\right.$16\%).

\section{4-Hydroxy-6-methyl-3-octyl-2H-pyran-2-one (7) ${ }^{3}$}

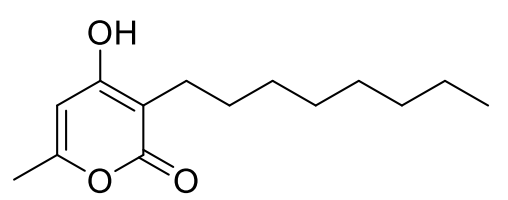

White solid $(0.211 \mathrm{~g}, 88 \%)$; m.p. $103-105^{\circ} \mathrm{C}$ (lit. $^{3} 103-$ 104.5 $\left.{ }^{\circ} \mathrm{C}\right) ;{ }^{1} \mathrm{H} \mathrm{NMR}\left(300 \mathrm{MHz}, \mathrm{CDCl}_{3}\right) \delta: 10.47$ (bs, $\left.1 \mathrm{H}\right), 6.25$ (s, 1H), 2.56-2.34 (m, 2H), $2.22(\mathrm{~s}, 3 \mathrm{H}), 1.61-1.15(\mathrm{~m}, 12 \mathrm{H}), 0.86$ $(\mathrm{t}, J=6.7 \mathrm{~Hz}, 3 \mathrm{H}) \mathrm{ppm} ;{ }^{13} \mathrm{C} \mathrm{NMR}\left(75 \mathrm{MHz}, \mathrm{CDCl}_{3}\right) \delta: 168.5,167.5,159.7,103.4,101.8,31.9$, 29.7, 29.6, 29.3, 28.1, 23.0, 22.7, 19.6, $14.1 \mathrm{ppm} ; \mathrm{m} / \mathrm{z}(\mathrm{ES}+): 239\left((\mathrm{M}+\mathrm{H})^{+} 98 \%\right)$.

\section{4-Hydroxy-6-methyl-3-nonyl-2H-pyran-2-one (8)}

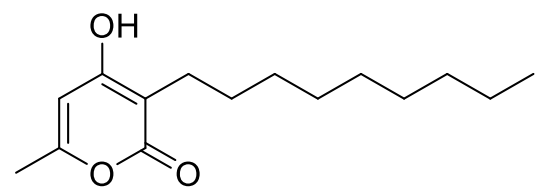

White solid (0.227 g, 90\%); m.p. 93-96 ${ }^{\circ} \mathrm{C}$; IR (film) $v_{\max }$ 3410, 1670, 1594, 1448, 1249, $1129 \mathrm{~cm}^{-1} ;{ }^{1} \mathrm{H}$ NMR (300 MHz, $\mathrm{CDCl}_{3}$ ) $\delta: 10.49$ (bs, 1H), 6.26 (s, 1H), 2.55-2.32 $(\mathrm{m}, 2 \mathrm{H}), 2.22(\mathrm{~s}, 3 \mathrm{H}), 1.61-1.11(\mathrm{~m}, 14 \mathrm{H}), 0.86(\mathrm{t}, J=6.7 \mathrm{~Hz}, 3 \mathrm{H}) \mathrm{ppm} ;{ }^{13} \mathrm{C} \mathrm{NMR}(75 \mathrm{MHz}$,

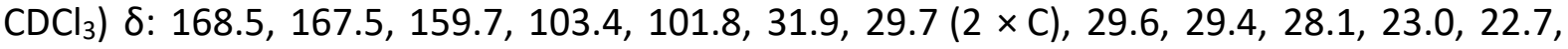
19.6, 14.1 ppm; HRMS (ESI-TOF) $m / z$ : $[\mathrm{M}+\mathrm{H}]^{+}$calcd for $\mathrm{C}_{15} \mathrm{H}_{25} \mathrm{O}_{3}$ : 253.1804; found: 253.1797; Anal. calcd for $\mathrm{C}_{15} \mathrm{H}_{24} \mathrm{O}_{3}$ : C, 71.39; $\mathrm{H}, 9.59$; found: $\mathrm{C}, 71.53 ; \mathrm{H}, 9.55$. 


\section{3-Decyl-4-hydroxy-6-methyl-2H-pyran-2-one (9) ${ }^{3}$}

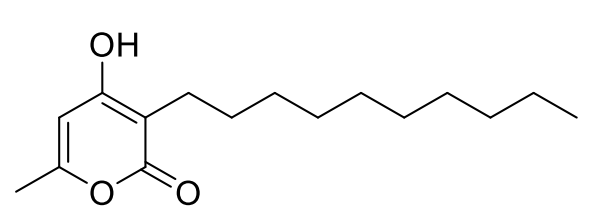

White solid (0.246 g, 92\%); m.p. $103-105^{\circ} \mathrm{C}$ (lit. ${ }^{3} 101.5-$ $\left.102.5^{\circ} \mathrm{C}\right) ;{ }^{1} \mathrm{H}$ NMR $\left(300 \mathrm{MHz}, \mathrm{CDCl}_{3}\right) \delta: 10.73$ (bs, $\left.1 \mathrm{H}\right)$, $6.29(\mathrm{~s}, 1 \mathrm{H}), 2.55-2.34(\mathrm{~m}, 2 \mathrm{H}), 2.22(\mathrm{~s}, 3 \mathrm{H}), 1.59-1.15$ (m, 16H), $0.87(\mathrm{t}, J=6.7 \mathrm{~Hz}, 3 \mathrm{H}) \mathrm{ppm} ;{ }^{13} \mathrm{C}$ NMR $\left(75 \mathrm{MHz}^{\mathrm{C}} \mathrm{CDCl}_{3}\right) \delta: 168.6,167.8,159.7,103.4$, 101.9, 31.9, 29.71, 29.67 ( $2 \times$ C), 29.64, 29.4, 28.1, 23.0, 22.7, 19.6, 14.1 ppm; m/z (ES+): 267 $\left((\mathrm{M}+\mathrm{H})^{+} 100 \%\right)$.

\section{4-Hydroxy-3-isobutyl-6-methyl-2H-pyran-2-one $(10)^{1}$}<smiles>Cc1cc(O)c(CC(C)C)c(=O)o1</smiles>
White solid (0.171 g, 94\%); m.p. $144-148^{\circ} \mathrm{C}$ (lit. ${ }^{1} 143-148{ }^{\circ} \mathrm{C}$ ); ${ }^{1} \mathrm{H}$ NMR (300 MHz, $\left.\mathrm{CDCl}_{3}\right)$ ): $10.34(\mathrm{~s}, 1 \mathrm{H}), 6.18(\mathrm{~s}, 1 \mathrm{H}), 2.28(\mathrm{~d}, \mathrm{~J}=7.3 \mathrm{~Hz}, 2 \mathrm{H}), 2.14$ (s, 3H), 1.96-1.79 (m, 1H), 0.85 (d, $J=6.7 \mathrm{~Hz}, 6 \mathrm{H}) \mathrm{ppm} ;{ }^{13} \mathrm{C}$ NMR $(75 \mathrm{MHz}$,

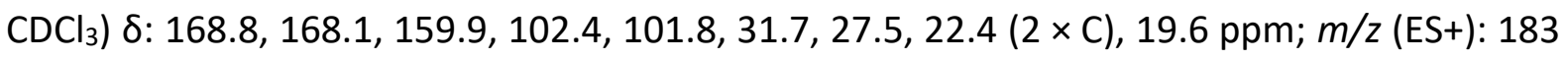
$\left((\mathrm{M}+\mathrm{H})^{+} 100 \%\right)$.

\section{4-Hydroxy-3-isopentyl-6-methyl-2H-pyran-2-one (11)}<smiles>Cc1cc(O)c(CCC(C)C)c(=O)o1</smiles>

White crystalline solid (0.185 g, 94\%); m.p. $149-155^{\circ} \mathrm{C}$; IR (film) $v_{\max }$ 3429, 1669, 1578, 1450, 1249, $1131 \mathrm{~cm}^{-1}$; ${ }^{1} \mathrm{H}$ NMR (300 MHz, $\left.\mathrm{CDCl}_{3}\right) \delta$ : 10.13 (bs, 1H), 6.22 (s, 1H), 2.50-2.37 (m, 2H), 2.22 (s, 3H), 1.68-1.49 (m, 1H), 1.47-1.29 (m, 2H), $0.92(\mathrm{~d}, J=6.6 \mathrm{~Hz}, 6 \mathrm{H}) \mathrm{ppm} ;{ }^{13} \mathrm{C} \mathrm{NMR}\left(75 \mathrm{MHz}, \mathrm{CDCl}_{3}\right)$ 8: 168.6, 167.6, 159.7, 103.6, 101.9, 37.0, 22.5 (2 ×C), 21.8, 21.0, 19.7 ppm; HRMS (ESI-TOF) m/z: $[\mathrm{M}+\mathrm{H}]^{+}$calcd for $\mathrm{C}_{11} \mathrm{H}_{17} \mathrm{O}_{3}$ : 197.1178; found: 197.1184 .

\section{3-Benzyl-4-hydroxy-6-methyl-2H-pyran-2-one (12) ${ }^{1}$}<smiles>Cc1cc(O)c(Cc2ccccc2)c(=O)o1</smiles>

White solid (0.204 g, 94\%); m.p. $167-170{ }^{\circ} \mathrm{C}$ (lit. $\left.{ }^{1} 156-158^{\circ} \mathrm{C}\right) ;{ }^{1} \mathrm{H}$ NMR

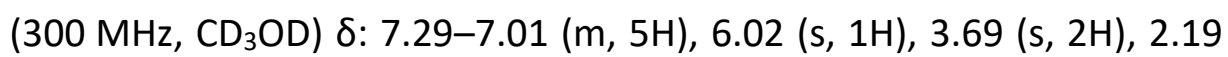

(s, 3H) ppm; ${ }^{13} \mathrm{C}$ NMR $\left(75 \mathrm{MHz}, \mathrm{CD}_{3} \mathrm{OD}\right) \delta: 168.5,168.0,162.3,141.7$, $129.4(2 \times \mathrm{C}), 129.0(2 \times \mathrm{C}), 126.8,103.0,101.4,29.5,19.5 \mathrm{ppm} ; \mathrm{m} / \mathrm{z}(\mathrm{ES}+): 217\left((\mathrm{M}+\mathrm{H})^{+}\right.$ $54 \%)$. 


\section{4-Hydroxy-6-methyl-3-(3-phenylpropyl)-2H-pyran-2-one (13) ${ }^{4}$}

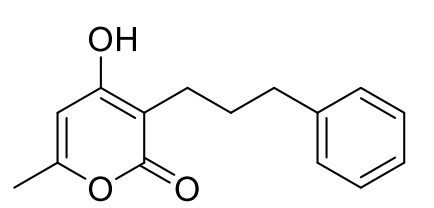

Off-white solid (0.220 g, 90\%); m.p. $129-130{ }^{\circ} \mathrm{C}$ (lit. $\left.{ }^{4} 129-131^{\circ} \mathrm{C}\right)$; ${ }^{1} \mathrm{H}$ NMR $\left(300 \mathrm{MHz}, \mathrm{CDCl}_{3}\right) \delta: 10.70$ (bs, $\left.1 \mathrm{H}\right), 7.36-6.98(\mathrm{~m}, 5 \mathrm{H})$, $6.25(\mathrm{~s}, 1 \mathrm{H}), 2.84-2.37(\mathrm{~m}, 4 \mathrm{H}), 2.16(\mathrm{~s}, 3 \mathrm{H}), 1.98-1.69(\mathrm{~m}, 2 \mathrm{H})$ ppm; ${ }^{13} \mathrm{C}$ NMR $\left(75 \mathrm{MHz}, \mathrm{CDCl}_{3}\right) \delta: 168.6,167.8,160.1,142.5,128.3(2 \times \mathrm{C}), 128.2(2 \times \mathrm{C})$, 125.6, 102.9, 101.9, 35.9, 29.7, 23.1, 19.7 ppm; m/z (ES+): $245\left((\mathrm{M}+\mathrm{H})^{+}\right.$100\%).

\section{4-Hydroxy-6-methyl-3-(2-phenylpropyl)-2H-pyran-2-one (14)}<smiles>Cc1cc(O)c(CC(C)c2ccccc2)c(=O)o1</smiles>

White solid $(0.223 \mathrm{~g}, 91 \%) ;$ m.p. $184-186^{\circ} \mathrm{C}$; IR (film) $v_{\max } 3389$, 1641, 1575, 1445, 1257, $1122 \mathrm{~cm}^{-1}$; ${ }^{1} \mathrm{H}$ NMR $\quad(300 \mathrm{MHz}$,

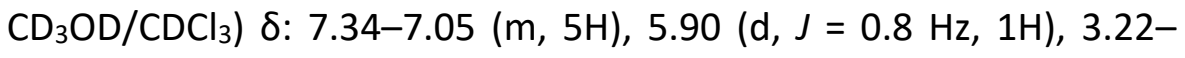
$3.04(\mathrm{~m}, 1 \mathrm{H}), 2.73-2.55(\mathrm{~m}, 2 \mathrm{H}), 2.18(\mathrm{~d}, J=0.8 \mathrm{~Hz}, 3 \mathrm{H}), 1.23(\mathrm{~d}, J=7.0 \mathrm{~Hz}, 3 \mathrm{H}) \mathrm{ppm}$; ${ }^{13} \mathrm{C} \mathrm{NMR}\left(75 \mathrm{MHz}, \mathrm{CD}_{3} \mathrm{OD} / \mathrm{CDCl}_{3}\right)$ 8: 168.4, 167.7, 161.0, 148.0, $128.6(2 \times \mathrm{C}), 127.6(2 \times \mathrm{C})$, 126.4, 102.0, 101.2, 38.8, 32.3, 21.3, 19.7 ppm; HRMS (ESI-TOF) $\mathrm{m} / \mathrm{z}:[\mathrm{M}+\mathrm{H}]^{+}$calcd for $\mathrm{C}_{15} \mathrm{H}_{17} \mathrm{O}_{3}$ : 245.1178; found: 245.1170 .

\section{4-Hydroxy-6-methyl-3-(pyridin-3-ylmethyl)-2H-pyran-2-one (15)}<smiles>Cc1cc(O)c(Cc2cccnc2)c(=O)o1</smiles>
Compound 10 precipitated out of the reaction mixture, was collected via suction filtration and recrystallised from hot $\mathrm{DCM} / \mathrm{MeOH}$ to yield a white crystalline solid $(0.076 \mathrm{~g}, 35 \%)$; m.p. $202-205^{\circ} \mathrm{C}$; IR (film) $V_{\max }$ $3382,1683,1661,1587,1448,1270,1120 \mathrm{~cm}^{-1} ;{ }^{1} \mathrm{H}$ NMR (300 MHz, $\left.\left(\mathrm{CD}_{3}\right)_{2} \mathrm{SO}\right)$ 8: 8.54-8.24 (m, 2H), $7.57(\mathrm{dt}, J=7.8,1.9 \mathrm{~Hz}, 1 \mathrm{H}), 7.26(\mathrm{dd}, J=7.8,4.8 \mathrm{~Hz}, 1 \mathrm{H}), 6.04(\mathrm{~s}, 1 \mathrm{H}), 3.59(\mathrm{~s}, 2 \mathrm{H})$, 2.16 (s, 3H) ppm; ${ }^{13} \mathrm{C}$ NMR $\left(75 \mathrm{MHz},\left(\mathrm{CD}_{3}\right)_{2} \mathrm{SO}\right) \delta: 166.4,165.2,161.2,149.9,147.5,136.6$, 136.0, 123.8, 100.3, 100.1, 26.2, $19.8 \mathrm{ppm}$; HRMS (ESI-TOF) $\mathrm{m} / \mathrm{z}$ : $[\mathrm{M}+\mathrm{H}]^{+}$calcd for $\mathrm{C}_{12} \mathrm{H}_{12} \mathrm{NO}_{3}$ : 218.0817; found: 218.0810; Anal. calcd for $\mathrm{C}_{12} \mathrm{H}_{11} \mathrm{NO}_{3}$ : C, 66.35; $\mathrm{H}, 5.10 ; \mathrm{N}, 6.45$; found: $C, 65.06 ; H, 5.18 ; N, 6.73$.

\section{3-(Furan-3-ylmethyl)-4-hydroxy-6-methyl-2H-pyran-2-one (16)}<smiles>Cc1cc(O)c(Cc2ccoc2)c(=O)o1</smiles>

Beige crystalline solid $(0.175 \mathrm{~g}, 85 \%)$; m.p. $187-189^{\circ} \mathrm{C}$; IR (film) $\mathrm{V}_{\max }$ 3401, 1663, 1619, 1572, 1448, 1262, 1124, $1018 \mathrm{~cm}^{-1}$; ${ }^{1} \mathrm{H}$ NMR (300 MHz, (CD $\left.)_{2} \mathrm{SO}\right)$ 8: 11.29 (bs, $\left.1 \mathrm{H}\right), 7.48$ (t, $\left.J=1.6 \mathrm{~Hz}, 1 \mathrm{H}\right), 7.34$ (d, $J=$ $0.5 \mathrm{~Hz}, 1 \mathrm{H}), 6.31(\mathrm{~d}, J=0.9 \mathrm{~Hz}, 1 \mathrm{H}), 6.02(\mathrm{~d}, J=0.7 \mathrm{~Hz}, 1 \mathrm{H}), 3.37(\mathrm{~s}, 2 \mathrm{H}), 2.15(\mathrm{~s}, 3 \mathrm{H}) \mathrm{ppm}$; 
${ }^{13} \mathrm{C}$ NMR $\left(75 \mathrm{MHz},\left(\mathrm{CD}_{3}\right)_{2} \mathrm{SO}\right)$ ): 165.3, 164.6, 160.3, 142.7, 139.1, 123.1, 111.4, 100.0, 99.8, 19.3, 18.1 ppm; HRMS (ESI-TOF) $\mathrm{m} / \mathrm{z}$ : [M+H] $]^{+}$calcd for $\mathrm{C}_{11} \mathrm{H}_{11} \mathrm{O}_{4}$ : 207.0657; found: 207.0653.

\section{3-(Furan-2-ylmethyl)-4-hydroxy-6-methyl-2H-pyran-2-one (17)}<smiles>Cc1cc(O)c(Cc2ccco2)c(=O)o1</smiles>

Yellow solid (0.194 g, 93\%); m.p. $172-174{ }^{\circ} \mathrm{C}$ (lit. $\left.{ }^{2} 177-178{ }^{\circ} \mathrm{C}\right) ;$ IR (film) $V_{\max } 3380,1664,1621,1573,1449,1263,1125,1020 \mathrm{~cm}^{-1} ;{ }^{1} \mathrm{H}$ NMR $\left(300 \mathrm{MHz},\left(\mathrm{CD}_{3}\right)_{2} \mathrm{SO}\right) \delta: 7.43$ (dd, $\left.J=1.8,0.8 \mathrm{~Hz}, 1 \mathrm{H}\right), 6.29$ (dd, $J=3.1$, $1.9 \mathrm{~Hz}, 1 \mathrm{H}$ ), 6.03 (d, J=0.9 Hz, 1H), 5.92 (dd, $J=3.1,0.9 \mathrm{~Hz}, 1 \mathrm{H}), 3.58$ (s, 2H), 2.16 (d, $J=0.7$ $\mathrm{Hz}, 3 \mathrm{H}$ ) ppm; ${ }^{13} \mathrm{C}$ NMR $\left(75 \mathrm{MHz},\left(\mathrm{CD}_{3}\right)_{2} \mathrm{SO}\right)$ 8: 166.0, 164.4, 160.7, 153.3, 140.9, 110.3, 105.0, 99.8, 97.3, 21.7, 19.3 ppm; HRMS (ESI-TOF) $\mathrm{m} / \mathrm{z}$ : $[\mathrm{M}+\mathrm{H}]^{+}$calcd for $\mathrm{C}_{11} \mathrm{H}_{11} \mathrm{O}_{4}$ : 207.0657; found: 207.0656 .

\section{Synthesis of 6-alkyl-4-hydroxy-2H-pyran-2-ones}

Representative procedure for electrophilic substitution at $\mathrm{C7}$ of 6-alkyl-4-hydroxy-2H-pyran-2-ones

Method A: A Schlenk tube was heated under vacuum and refilled with $\mathrm{N}_{2}$ three times. 2-Pyrone (1.0 equiv.) and HMDS (3 $\mathrm{mL} / \mathrm{mmol})$ were added, and the resulting reaction mixture was heated to $80^{\circ} \mathrm{C}$ under $\mathrm{N}_{2}$ for 1 hour. The solution was allowed to cool and the HMDS removed under reduced pressure. THF $(3 \mathrm{~mL} / \mathrm{mmol})$ was then added, and the solution cooled to $-78^{\circ} \mathrm{C}$. $n$-BuLi (1.25 equiv.) was added carefully over $15 \mathrm{~min}$., and the solution stirred for 1 hour. Alkyl bromide (2.3 equiv.) was then added over $10 \mathrm{~min}$. and the solution allowed to warm gradually to r.t., then stirred for 16 hours. The reaction was then quenched with $6 \mathrm{M} \mathrm{HCl}$ until $\mathrm{pH} \sim 2$ and the solvent was concentrated under reduced pressure. The residual mass was dissolved in ethyl acetate $(10 \mathrm{~mL})$ and washed with brine (2 $\times 10 \mathrm{~mL}$ ). The combined organic extracts were dried over MgSO4, filtered and concentrated under reduced pressure. The crude product was purified by column chromatography (hexanes:EtOAc 1:1). ${ }^{5}$

\section{4-Hydroxy-6-octyl-2H-pyran-2-one (20) ${ }^{6}$}

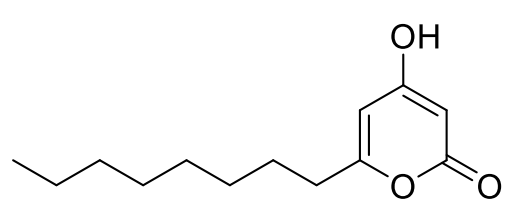

White solid $(0.046 \mathrm{~g}, 10 \%)$; m.p. $56-59^{\circ} \mathrm{C}$ (lit. ${ }^{6} 57-59^{\circ} \mathrm{C}$ );

${ }^{1} \mathrm{H}$ NMR (300 MHz, CDCl 3 ) $\delta: 10.91$ (br s, 1H), 5.97 (d, J = 1.5

$\mathrm{Hz}, 1 \mathrm{H}), 5.57(\mathrm{~d}, J=1.8 \mathrm{~Hz}, 1 \mathrm{H}), 2.47(\mathrm{t}, J=7.5 \mathrm{~Hz}, 2 \mathrm{H}), 1.64$ 
(t, $J=7.2 \mathrm{~Hz}, 2 \mathrm{H}), 1.41-1.14(\mathrm{~m}, 10 \mathrm{H}), 0.88(\mathrm{t}, J=6.7 \mathrm{~Hz}, 3 \mathrm{H}) . \mathrm{ppm} ;{ }^{13} \mathrm{C} \mathrm{NMR}\left(75 \mathrm{MHz}, \mathrm{CDCl}_{3}\right)$

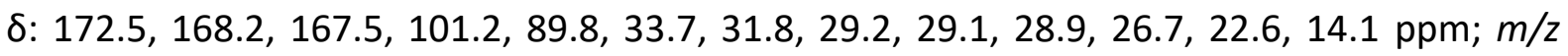
(ES-): $223\left((\mathrm{M}-\mathrm{H})^{-}\right.$14\%).

\section{4-Hydroxy-6-nonyl-2H-pyran-2-one (21) ${ }^{7}$}

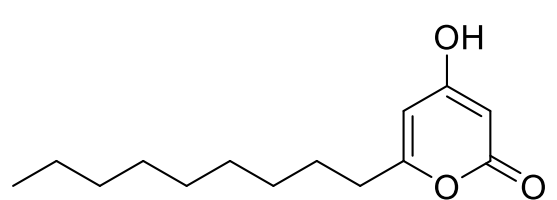

White solid $(0.060$ g, $13 \%)$; m.p. $79-80^{\circ} \mathrm{C}$ (lit. $\left.78-79^{\circ} \mathrm{C}\right)$; ${ }^{1} \mathrm{H}$ NMR $\left(300 \mathrm{MHz}, \mathrm{CDCl}_{3}\right) \delta: 10.83$ (br s, 1H), 5.97 (d, $J=$ $1.3 \mathrm{~Hz}, 1 \mathrm{H}), 5.58(\mathrm{~d}, J=1.7 \mathrm{~Hz}, 1 \mathrm{H}), 2.47(\mathrm{t}, J=7.4 \mathrm{~Hz}, 2 \mathrm{H})$, $1.64(\mathrm{t}, J=7.4 \mathrm{~Hz}, 2 \mathrm{H}), 1.43-1.14(\mathrm{~m}, 12 \mathrm{H}), 0.88(\mathrm{t}, J=6.4 \mathrm{~Hz}, 3 \mathrm{H}) \mathrm{ppm} ;{ }^{13} \mathrm{C}$ NMR $(75 \mathrm{MHz}$,

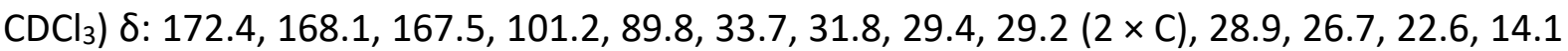
ppm; $m / z$ (ES-): 237 ((M-H)- 100\%).

Method B: A Schlenk tube was heated under vacuum and refilled with $\mathrm{N}_{2}$ three times. 2-Pyrone (1.0 equiv.) was added, followed by THF $(2.77 \mathrm{~mL} / \mathrm{mmol})$, the resulting white suspension was stirred at r.t. for $5 \mathrm{~min}$. TMEDA $(1.0 \mathrm{mmol}, 1.0$ equiv.) and HMPA $(0.55 \mathrm{~mL} / \mathrm{mmol})$ were added, and the resulting pale-yellow reaction mixture was cooled to $0{ }^{\circ} \mathrm{C}$ for $30 \mathrm{~min}$. $n$-BuLi (2.4 equiv.) was added dropwise over $10 \mathrm{~min}$. giving a deep red reaction mixture that was stirred for a further $1 \mathrm{~h}$ at $0{ }^{\circ} \mathrm{C}$, followed by the dropwise addition the corresponding alkyl iodide (1.8 equiv.). The orange reaction mixture was warmed to r.t. and stirred under $\mathrm{N}_{2}$ for $16 \mathrm{~h}$. The reaction was acidified with $4 \mathrm{M} \mathrm{HCl}$ until $\mathrm{pH} \sim 2-3$ and was then extracted with $\mathrm{Et}_{2} \mathrm{O}(3 \times 10 \mathrm{~mL})$. The combined organic layers were washed with $\mathrm{H}_{2} \mathrm{O}(3 \times 10 \mathrm{~mL})$ and brine $(10 \mathrm{~mL})$, dried over $\mathrm{MgSO}_{4}$, filtered and concentrated under reduced pressure. The crude product was purified by column chromatography (hexanes:EtOAc 7:3). ${ }^{1}$

\section{3-Hexyl-4-hydroxy-6-isobutyl-2H-pyran-2-one (1) ${ }^{1}$}

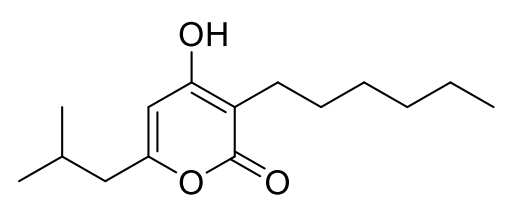

Pale yellow solid (0.062 g, 49\%); m.p. $109-112^{\circ} \mathrm{C} ;{ }^{1} \mathrm{H}$ NMR $\left(300 \mathrm{MHz}, \mathrm{CDCl}_{3}\right)$ 8: 10.49 (bs, 1H), 6.21 (s, 1H), 2.58-2.21 $(\mathrm{m}, 4 \mathrm{H}), 2.15-1.94(\mathrm{~m}, 1 \mathrm{H}), 1.55-1.22(\mathrm{~m}, 8 \mathrm{H}), 1.01-0.75(\mathrm{~m}$, 9H) ppm; ${ }^{13} \mathrm{C} \mathrm{NMR}\left(75 \mathrm{MHz}, \mathrm{CDCl}_{3}\right) \delta: 168.4,167.4,162.6,103.5,102.0,42.6,31.8,29.1$, 28.0, 26.2, 23.1, 22.7, $22.2(2 \times \mathrm{C}), 14.1 \mathrm{ppm} ; \mathrm{m} / \mathrm{z}(\mathrm{ES}+)$ : $253\left((\mathrm{M}+\mathrm{H})^{+}\right.$100\%); Anal. calcd. for $\mathrm{C}_{15} \mathrm{H}_{24} \mathrm{O}_{3}$ : C, 71.39; $\mathrm{H}, 9.59$; found: $\mathrm{C}, 71.32 ; \mathrm{H}, 9.39$. 


\section{6-Hexyl-4-hydroxy-2H-pyran-2-one $(18)^{8}$}

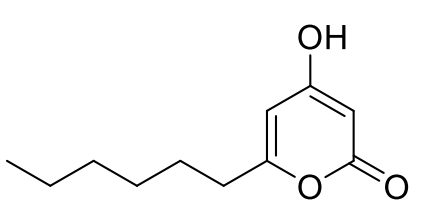

Yellow-orange solid $(0.034$ g, $17 \%)$; m.p. $44-46^{\circ} \mathrm{C}$ (lit. $\left.{ }^{8} 56-57^{\circ} \mathrm{C}\right)$;

${ }^{1} \mathrm{H}$ NMR $\left(300 \mathrm{MHz}, \mathrm{CDCl}_{3}\right) \delta: 6.00(\mathrm{~s}, 1 \mathrm{H}), 5.58(\mathrm{bs}, 1 \mathrm{H}), 2.47(\mathrm{t}, J=$ $7.6 \mathrm{~Hz}, 2 \mathrm{H}), 1.76-1.53(\mathrm{~m}, 2 \mathrm{H}), 1.40-1.19(\mathrm{~m}, 6 \mathrm{H}), 0.88(\mathrm{t}, J=6.7$ $\mathrm{Hz}, 3 \mathrm{H}) \mathrm{ppm} ;{ }^{13} \mathrm{CNMR}\left(75 \mathrm{MHz} \mathrm{CDCl}_{3}\right) \delta: 172.7,168.4,167.3,101.3,89.8,33.6,31.4,28.6$, 26.6, 22.4, $14.0 \mathrm{ppm} ; \mathrm{m} / \mathrm{z}(\mathrm{ES}+): 197\left((\mathrm{M}+\mathrm{H})^{+}\right.$70\%).

\section{6-Heptyl-4-hydroxy-2H-pyran-2-one (19) ${ }^{9}$}

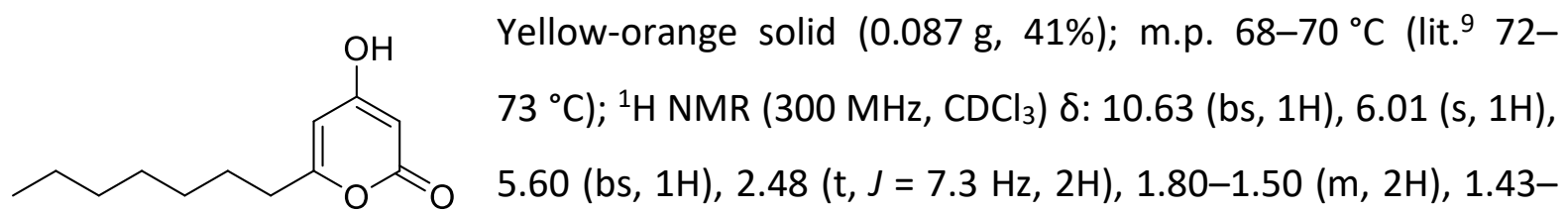
$1.15(\mathrm{~m}, 8 \mathrm{H}), 0.87(\mathrm{t}, J=6.4 \mathrm{~Hz}, 3 \mathrm{H}) \mathrm{ppm} ;{ }^{13} \mathrm{C} \mathrm{NMR}\left(75 \mathrm{MHz}, \mathrm{CDCl}_{3}\right) \delta: 172.8,167.3,166.9$, 101.4, 89.8, 33.6, 31.6, $28.9(2 \times C), 26.7,22.6,14.0$ ppm; m/z (ES+): $211\left((M+H)^{+} 100 \%\right)$.

\section{4-Hydroxy-6-isobutyl-2H-pyran-2-one $(24)^{1}$}

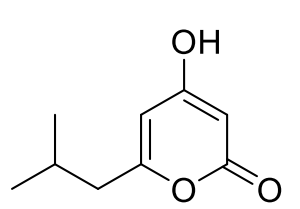

White solid (0.165 g, 58\%); m.p. $103-106^{\circ} \mathrm{C}$ (lit. ${ }^{10} 106-108{ }^{\circ} \mathrm{C}$ ); ${ }^{1} \mathrm{H}$ NMR

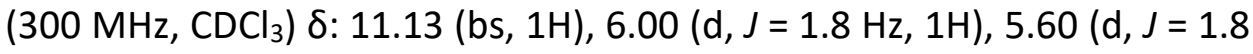
$\mathrm{Hz}, 1 \mathrm{H}), 2.35(\mathrm{~d}, J=7.2 \mathrm{~Hz}, 2 \mathrm{H}), 2.15-1.94(\mathrm{~m}, 1 \mathrm{H}), 0.95(\mathrm{~d}, J=6.6 \mathrm{~Hz}, 6 \mathrm{H})$ ppm; ${ }^{13} \mathrm{CNMR}\left(75 \mathrm{MHz}, \mathrm{CDCl}_{3}\right.$ ) $\delta: 172.6,168.5,166.4,102.4,89.9,42.8,26.9,22.2(2 \times \mathrm{C})$ ppm; $m / z$ (ES-): 167 ((M-H)- 100\%).

\section{4-Hydroxy-6-isobutyl-3-octyl-2H-pyran-2-one (25)}

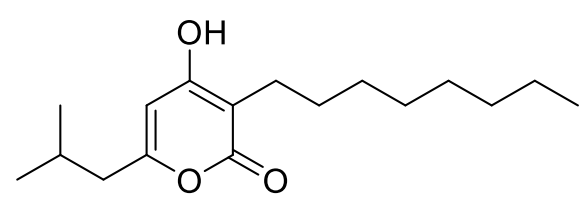

White solid $(0.063 \mathrm{~g}, 45 \%) ;$ m.p. $105-107^{\circ} \mathrm{C}$; IR (film) $V_{\max } 3089,1663,1569,1433,1273,1130 \mathrm{~cm}^{-1} ;{ }^{1} \mathrm{H}$ NMR (300 MHz, $\mathrm{CDCl}_{3}$ ) $\delta: 9.58$ (bs, $\left.1 \mathrm{H}\right), 6.13(\mathrm{~s}, 1 \mathrm{H}), 2.57-2.37$ (m, 2H), $2.30(\mathrm{~d}, J=7.2 \mathrm{~Hz}, 2 \mathrm{H}), 2.16-1.92(\mathrm{~m}, 1 \mathrm{H}), 1.59-1.15(\mathrm{~m}, 12 \mathrm{H}), 1.01-0.75(\mathrm{~m}, 9 \mathrm{H})$ ppm; ${ }^{13} \mathrm{CNMR}\left(75 \mathrm{MHz}, \mathrm{CDCl}_{3}\right.$ ) $\delta: 168.4,167.1,162.6,103.5,101.5,42.6,31.9,29.7,29.6$, 29.4, 28.1, 26.9, 23.1, 22.7, $22.2(2 \times C), 14.1$ ppm; HRMS (ESI-TOF) $\mathrm{m} / \mathrm{z}$ : $[\mathrm{M}+\mathrm{H}]^{+}$calcd. for $\mathrm{C}_{17} \mathrm{H}_{29} \mathrm{O}_{3}$ : 281.2111; found: 281.2113. 


\section{4-Hydroxy-6-isobutyl-3-nonyl-2H-pyran-2-one (26)}

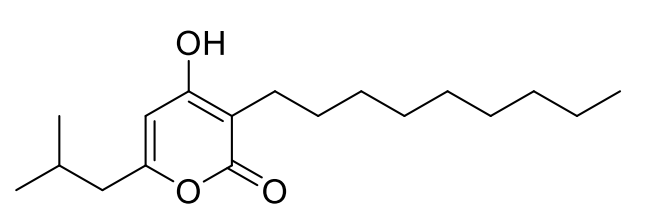

White solid (0.062 g, 42\%); m.p. $98-100{ }^{\circ} \mathrm{C}$; IR (film) $V_{\max }$ 3044, 1663, 1569, 1433, 1265, $1130 \mathrm{~cm}^{-1}$; ${ }^{1} \mathrm{H}$ NMR $\left(300 \mathrm{MHz}, \mathrm{CDCl}_{3}\right)$ 8: 9.51 (bs, $\left.1 \mathrm{H}\right), 6.12$ (s, $1 \mathrm{H}), 2.57-2.37(\mathrm{~m}, 2 \mathrm{H}), 2.30(\mathrm{~d}, \mathrm{~J}=7.2 \mathrm{~Hz}, 2 \mathrm{H}), 2.17-1.92(\mathrm{~m}, 1 \mathrm{H}), 1.64-1.15(\mathrm{~m}, 14 \mathrm{H}), 1.04-$ $0.66(\mathrm{~m}, 9 \mathrm{H}) \mathrm{ppm} ;{ }^{13} \mathrm{C} \mathrm{NMR}\left(75 \mathrm{MHz}, \mathrm{CDCl}_{3}\right)$ 8: 168.5, 167.3, 162.6, 103.5, 102.0, 42.6, 31.9, 29.74, 29.66 ( $2 \times$ C), 29.4, 28.1, 26.9, 23.1, 22.7, 22.2 ( $2 \times$ C), 14.1 ppm; HRMS (ESI-TOF) m/z: $[\mathrm{M}+\mathrm{H}]^{+}$calcd. for $\mathrm{C}_{18} \mathrm{H}_{31} \mathrm{O}_{3}: 295.2268$; found: 295.2268 .

\section{3-Decyl-4-hydroxy-6-isobutyl-2H-pyran-2-one (27)}

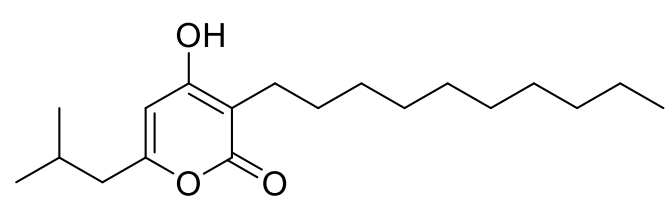

Pale yellow solid $(0.057 \mathrm{~g}, 37 \%)$; m.p. $95-96{ }^{\circ} \mathrm{C}$; IR (film) $V_{\max } 3090,1663,1567,1432,1258,1130 \mathrm{~cm}^{-}$ 1; ${ }^{1} \mathrm{H}$ NMR (300 MHz, $\left.\mathrm{CDCl}_{3}\right)$ 8: 9.08 (bs, $\left.1 \mathrm{H}\right), 6.08$ (s, 1H), 2.53-2.37 (m, 2H), $2.30(\mathrm{~d}, J=7.2 \mathrm{~Hz}, 2 \mathrm{H}), 2.16-1.96(\mathrm{~m}, 1 \mathrm{H}), 1.60-1.12(\mathrm{~m}, 16 \mathrm{H})$, 0.99-0.79 (m, 9H) ppm; $\left.{ }^{13} \mathrm{C} \mathrm{NMR} \mathrm{(75} \mathrm{MHz,} \mathrm{CDCl}_{3}\right)$ 8: 168.4, 167.1, 162.6, 103.5, 101.9, 42.6, $31.9,29.73,29.71,29.67,29.66,29.4,28.1,26.9,23.1,22.7,22.2$ ( $2 \times C), 14.1$ ppm; HRMS (ESI-TOF) $m / z:[\mathrm{M}+\mathrm{H}]^{+}$calcd. for $\mathrm{C}_{19} \mathrm{H}_{33} \mathrm{O}_{3}: 309.2424$; found: 309.2425 .

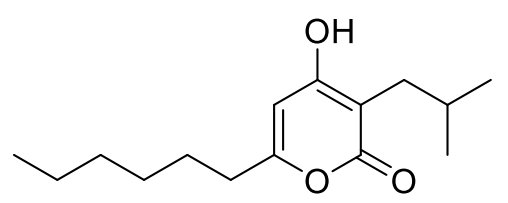

\section{6-Hexyl-4-hydroxy-3-isobutyl-2H-pyran-2-one (28)}

Pale yellow solid $(0.071 \mathrm{~g}, 56 \%) ;$ m.p. $105-108^{\circ} \mathrm{C}$; IR (film) $V_{\max } 3078,1664,1573,1435,1265,1124 \mathrm{~cm}^{-1} ;{ }^{1} \mathrm{H}$ NMR $(300$

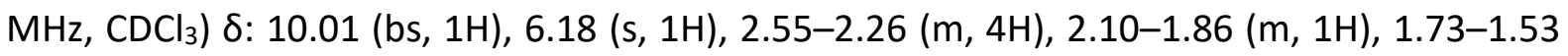
$(\mathrm{m}, 2 \mathrm{H}), 1.44-1.16(\mathrm{~m}, 6 \mathrm{H}), 1.03-0.79(\mathrm{~m}, 9 \mathrm{H}) \mathrm{ppm} ;{ }^{13} \mathrm{CNMR}\left(75 \mathrm{MHz}, \mathrm{CDCl}_{3}\right) \delta: 168.4$, $167.5,163.7,102.4,100.7,33.5,31.9,31.4,28.7,27.5,26.7,22.45,22.44$ (2 × C), 14.0 ppm; HRMS (ESI-TOF) m/z: [M+H] ${ }^{+}$calcd. for $\mathrm{C}_{15} \mathrm{H}_{25} \mathrm{O}_{3}$ : 253.1798; found: 253.1800 .

\section{Synthesis of 6-alkyl-4-hydroxy-pyridin-2(1H)-ones}

Representative procedure for the synthesis of 6-alkyl-4-hydroxy-pyridin-2(1H)-ones

The corresponding pyrone (1.0 equiv.), ammonia $(5 \mathrm{~mL} / \mathrm{mmol})$ and water $(2 \mathrm{~mL} / \mathrm{mmol})$ were heated at $130{ }^{\circ} \mathrm{C}$ for 6 hours. The reaction was cooled to r.t. and diluted with water $(4 \mathrm{~mL} / \mathrm{mmol})$. The mixture was acidified to $\mathrm{pH} \sim 1$ with $0.5 \mathrm{M} \mathrm{HCl}$ and the resulting precipitate was filtered and dried under vacuum. ${ }^{11}$ 


\section{4-Hydroxy-6-octylpyridin-2(1H)-one $(22)^{12}$}

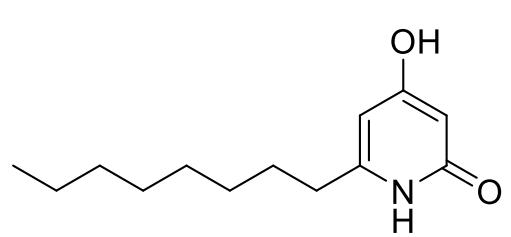

White solid $(0.010 \mathrm{~g}, 67 \%) ;$ m.p. $174-176^{\circ} \mathrm{C}$; ${ }^{1} \mathrm{H}$ NMR (500 MHz, $\left.\left(\mathrm{CD}_{3}\right)_{2} \mathrm{SO}\right) \delta: 10.86$ (br s, $\left.1 \mathrm{H}\right), 10.30$ (br s, 1H), $5.58(\mathrm{~d}, J=2.2 \mathrm{~Hz}, 1 \mathrm{H}), 5.33(\mathrm{~d}, J=2.2 \mathrm{~Hz}, 1 \mathrm{H}), 2.33$ (t, $J=7.6$ $\mathrm{Hz}, 2 \mathrm{H}), 1.60-1.42(\mathrm{~m}, 2 \mathrm{H}), 1.34-1.15(\mathrm{~m}, 10 \mathrm{H}), 0.86(\mathrm{t}, J=$ $6.9 \mathrm{~Hz}, 3 \mathrm{H}) \mathrm{ppm} ;{ }^{13} \mathrm{C} \mathrm{NMR}\left(125 \mathrm{MHz},\left(\mathrm{CD}_{3}\right)_{2} \mathrm{SO}\right)$ 8: 167.9, 165.2, 150.5, 97.8, 96.4, 32.6, 31.7, 29.1, 29.0, 28.8, 28.5, 22.5, 14.4 ppm; $m / z(E S+): 224\left((\mathrm{M}+\mathrm{H})^{+} 56 \%\right)$.

\section{4-Hydroxy-6-nonylpyridin-2(1H)-one $(23)^{12}$}

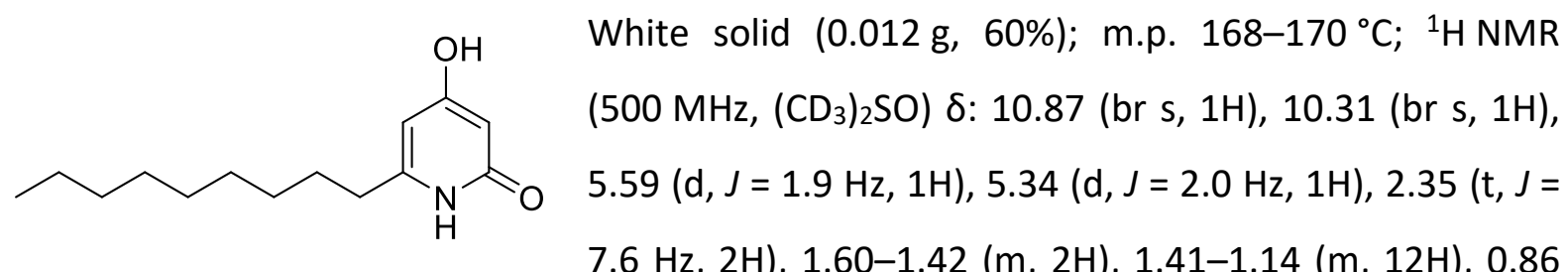

$(\mathrm{t}, J=6.8 \mathrm{~Hz}, 3 \mathrm{H}) \mathrm{ppm} ;{ }^{13} \mathrm{C} \mathrm{NMR}\left(125 \mathrm{MHz},\left(\mathrm{CD}_{3}\right)_{2} \mathrm{SO}\right) \delta: 167.9,165.2,150.5,97.8,96.4,32.5$, $31.7,29.3,29.11,29.10,28.8,28.5,22.5,14.4$ ppm; $m / z(E S+): 238\left((M+H)^{+} 82 \%\right)$. 


\section{Biological Figures and Tables}

(a)

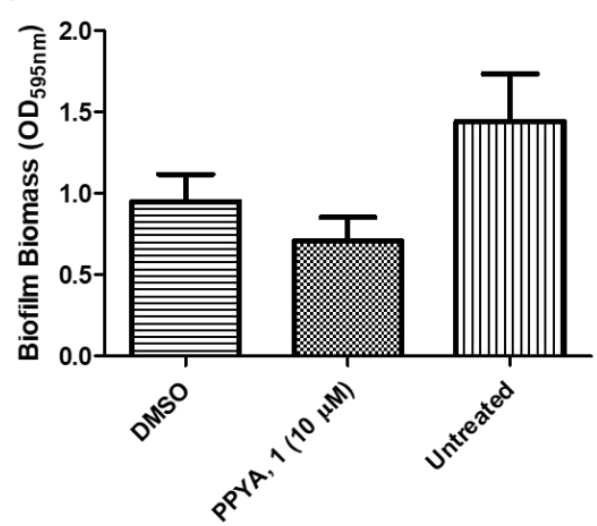

(b)
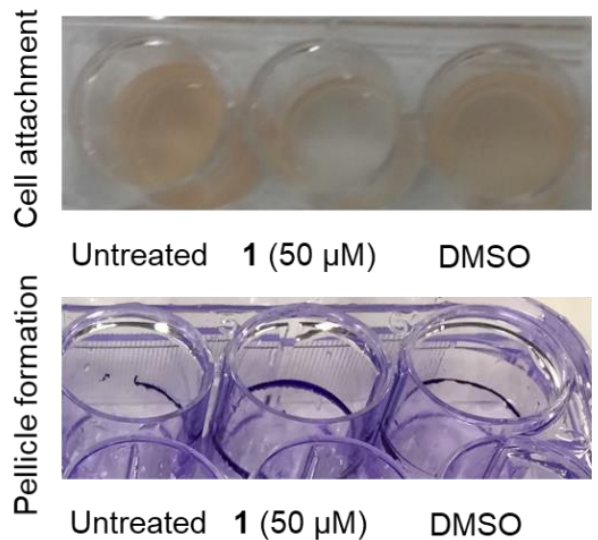

(c)

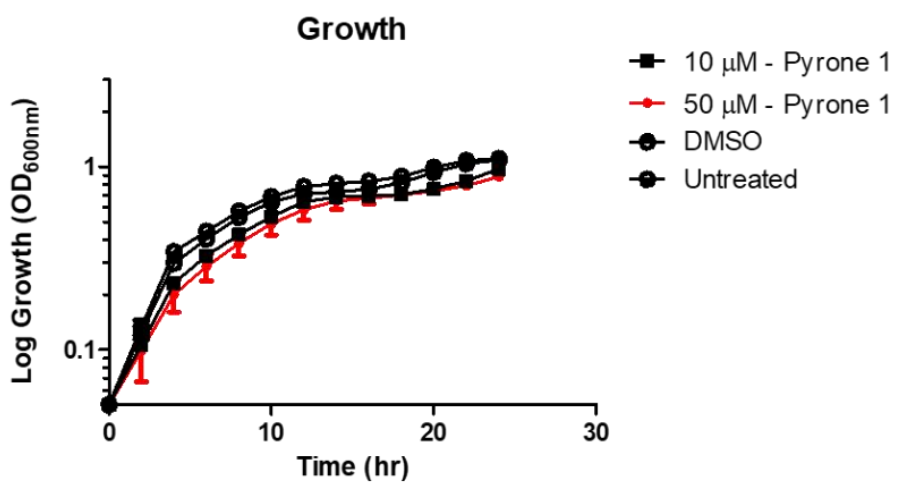

Figure S1 (a) Biofilm formation of B. atropheaus in the presence of $10 \mu M$ PPYA or DMSO control. Data represents the average (+/-SEM) of three independent biological replicates. (b) Pellicle visualisation of $B$. atropheaus biofilms formed on 24-well plates in the presence of (left to right: untreated, $10 \mu \mathrm{M}$ PPYA, DMSO) prior to quantification. Increased pellicle formation is evident in the PPYA wells when compared to the untreated and DMSO controls. (c) Growth kinetics of B. atropheaus in the presence of PPYA revealed no inhibitory activity suggesting that the phenotypes affected in the presence of this signal are the result of a mechanistic response. 


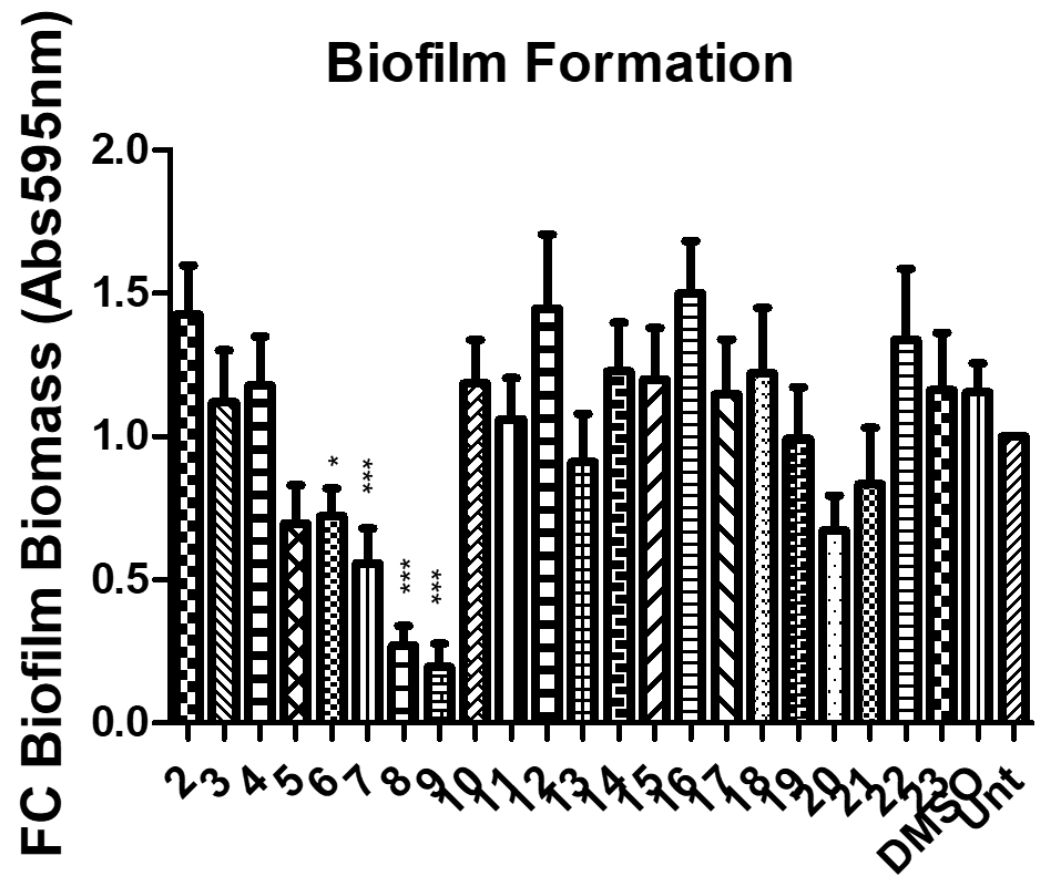

(b)

\section{Growth Kinetics}

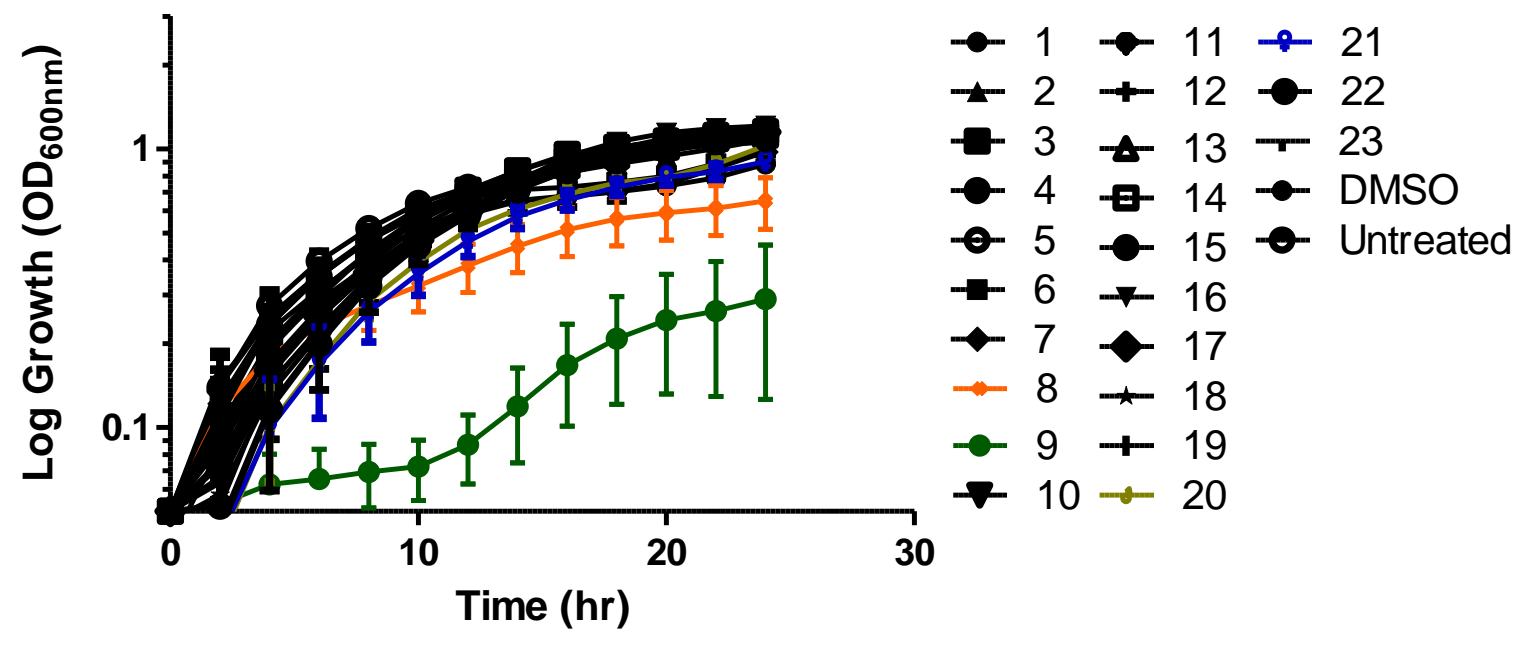

Figure S2 (a) Biofilm formation of B. atropheaus in the presence of pyrone derivative compounds. Assays performed in 96-well microtitre plates. Data presented is the average (+/- SEM) of at least three independent biological replicates. Statistical analysis was performed by One way ANOVA with Bonferroni Multiple Comparison post-hoc corrective testing (* $\left.{ }^{*} \leq 0.05,{ }^{* *} p \leq 0.001\right)$. (b) Growth kinetics analysis of B. atropheaus in the presence of $50 \mu \mathrm{M}$ pyrone signal or derivative compounds. Data presented is the average (+/- SEM) of three independent biological replicates. 
(a)

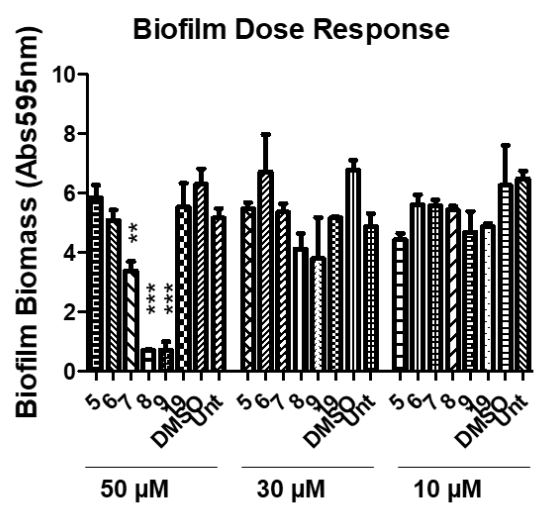

(c)

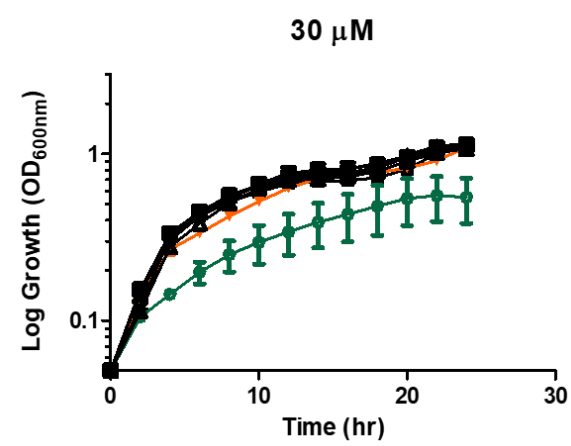

(b)

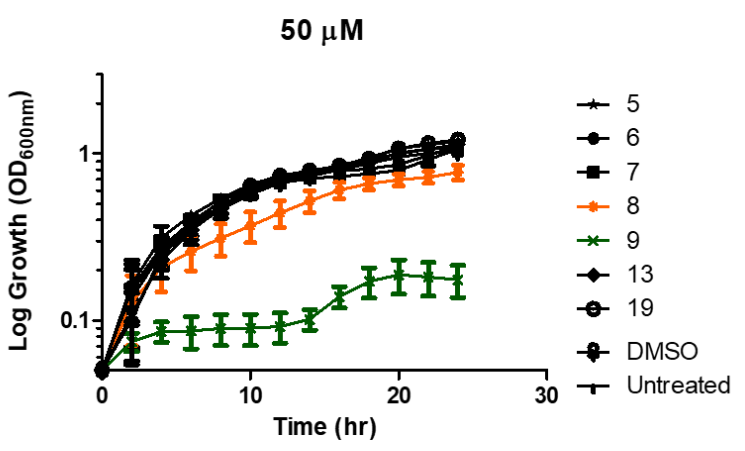

(d)

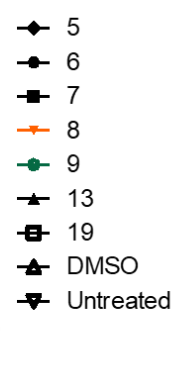

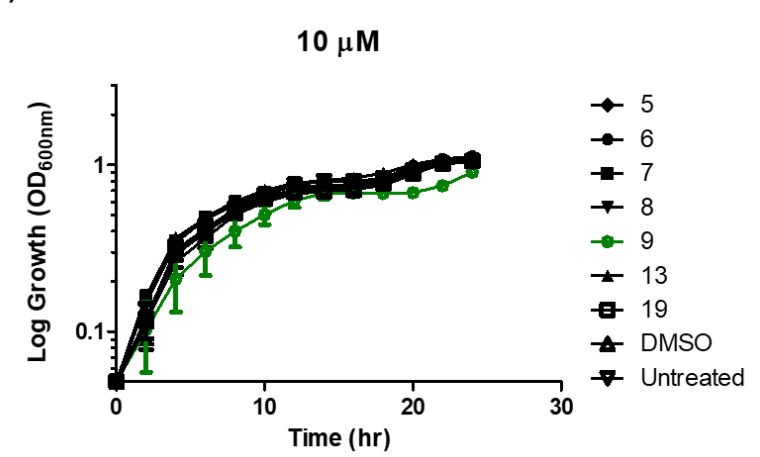

Figure S3 (a) Biofilm dose response of B. atropheaus in the presence of 50, 30, or $10 \mu \mathrm{M}$ pyrone derivatives. Data indicates a dose dependent response whereby antibiofilm activity is lost below a concentration of $50 \mu M$. (b-d) Growth kinetic analysis of B. atropheaus in the presence of 50, 30, or $10 \mu M$ pyrone derivatives. All data presented is the average (+/- SEM) of at least three independent biological replicates. Statistical analysis was performed by One way ANOVA with Bonferroni Multiple Comparison post-hoc corrective testing $\left({ }^{* *} p \leq 0.005\right.$, *** $p \leq 0.001)$. 


\section{${ }^{1} \mathrm{H}$ and ${ }^{13} \mathrm{C}$ NMR Spectra}

i<smiles>CCCCCCc1c(O)cc(CC(C)C)oc1=O</smiles>

${ }^{1} \mathrm{H}$ NMR $\left(300 \mathrm{MHz}, \mathrm{CDCl}_{3}\right)$

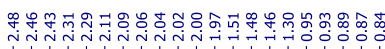

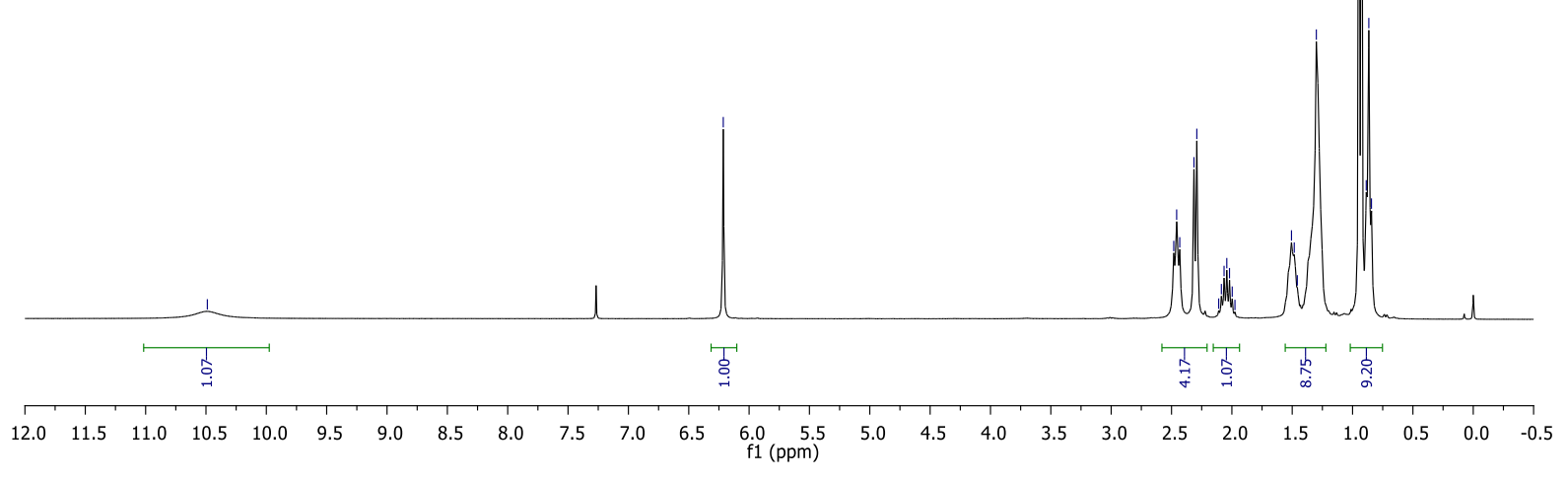

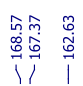

동ำ

|

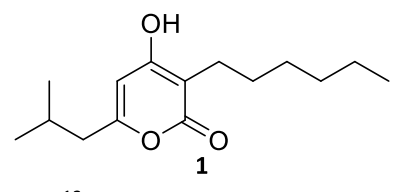

${ }^{13} \mathrm{C}$ NMR $\left(75 \mathrm{MHz}, \mathrm{CDCl}_{3}\right)$

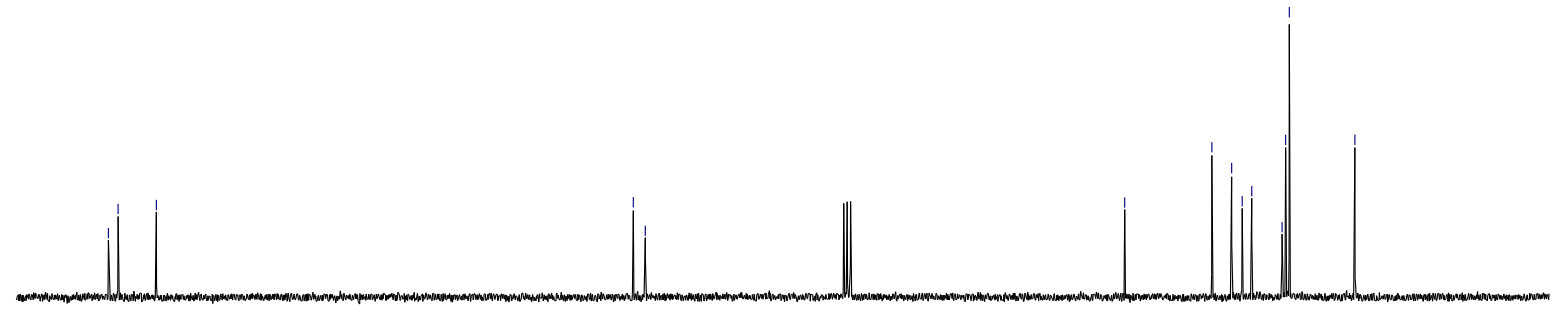

$\begin{array}{llllllllll}180 & 170 & 160 & 150 & 140 & 130 & 120 & 110 & 100 & 90 \\ & 121(\mathrm{ppm})\end{array}$

Figure S4: ${ }^{1} \mathrm{H} N M R$ (top) and ${ }^{13} \mathrm{C} N M R$ (bottom) spectra for compound 1. 


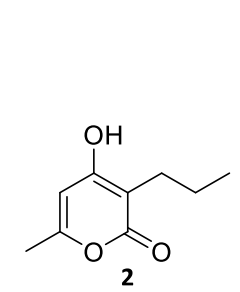

${ }^{1} \mathrm{H}$ NMR $\left(300 \mathrm{MHz}, \mathrm{CDCl}_{3}\right)$

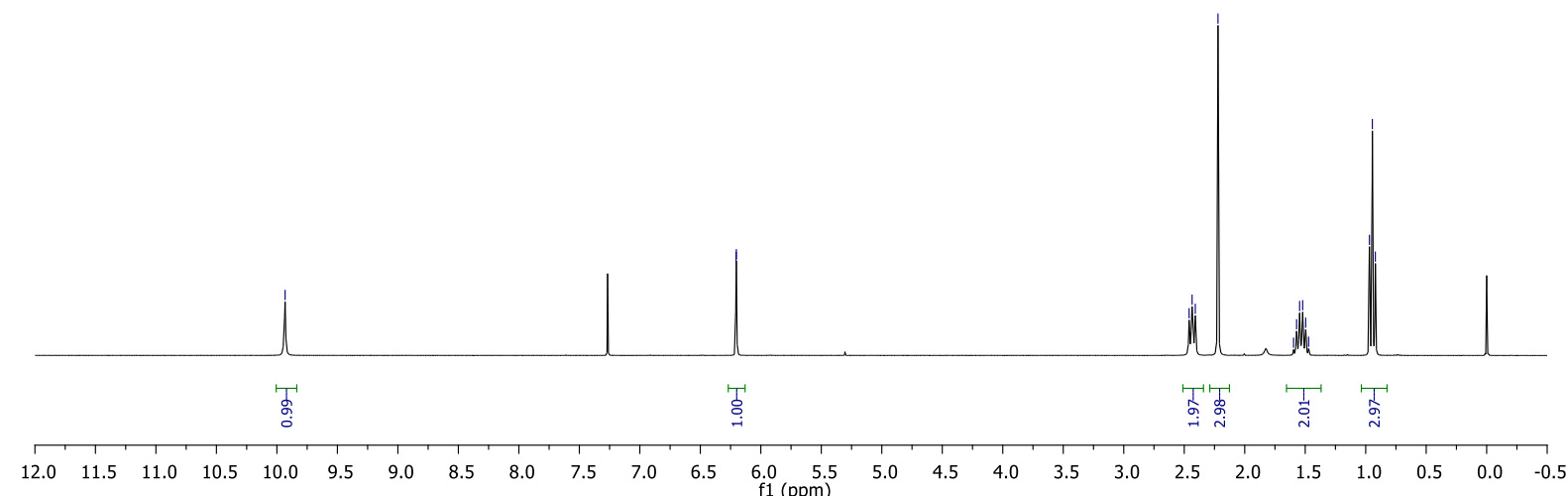

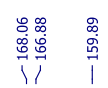

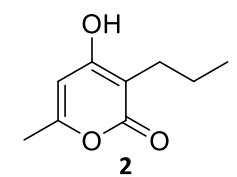

${ }^{13} \mathrm{CNMR}\left(75 \mathrm{MHz}, \mathrm{CDCl}_{3}\right.$ )
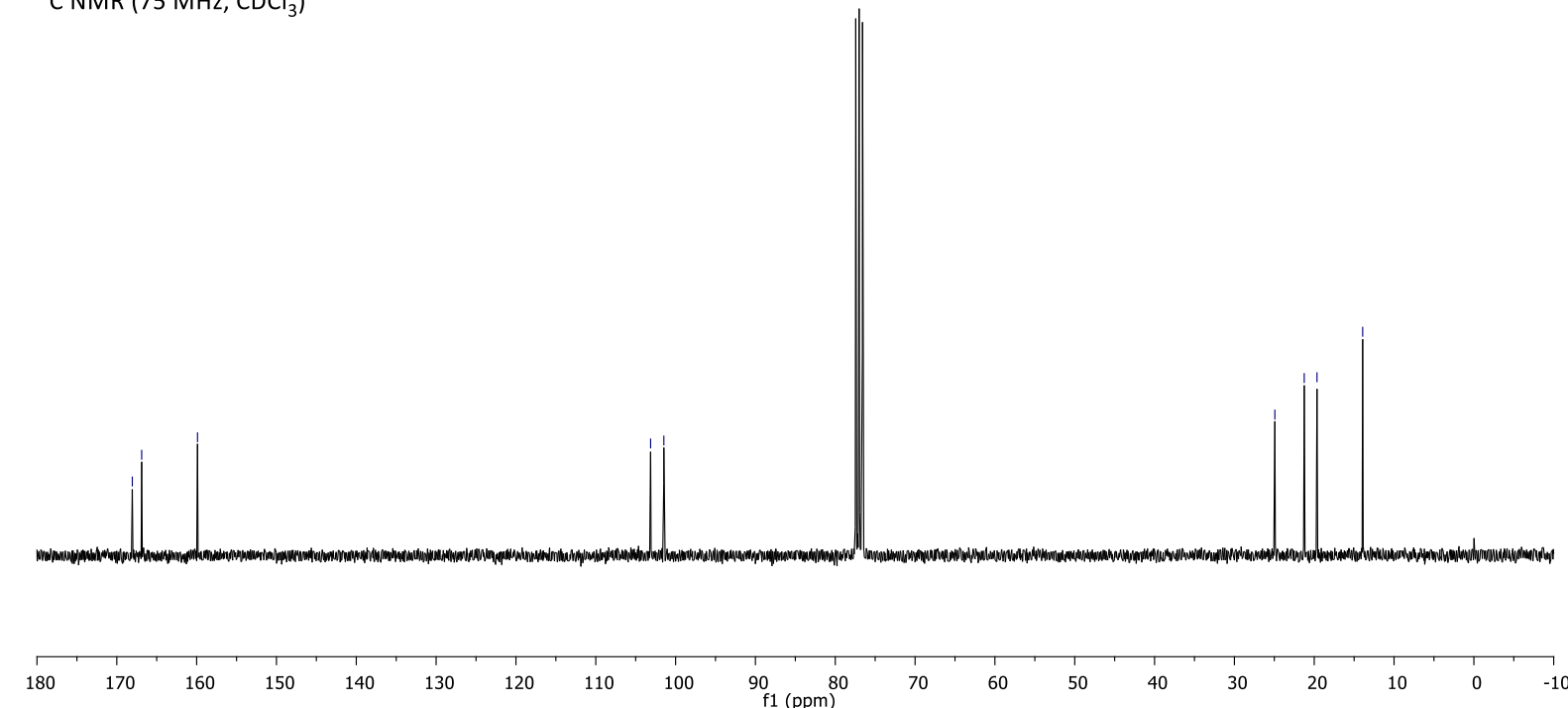

Figure S5: ${ }^{1} \mathrm{H} N M R$ (top) and ${ }^{13} \mathrm{C} N M R$ (bottom) spectra for compound 2. 


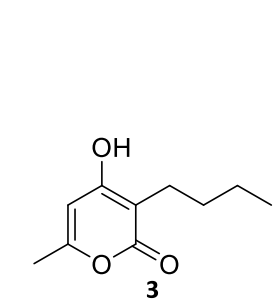

${ }^{1} \mathrm{H}$ NMR (300 MHz, CDCl ${ }_{3}$ )

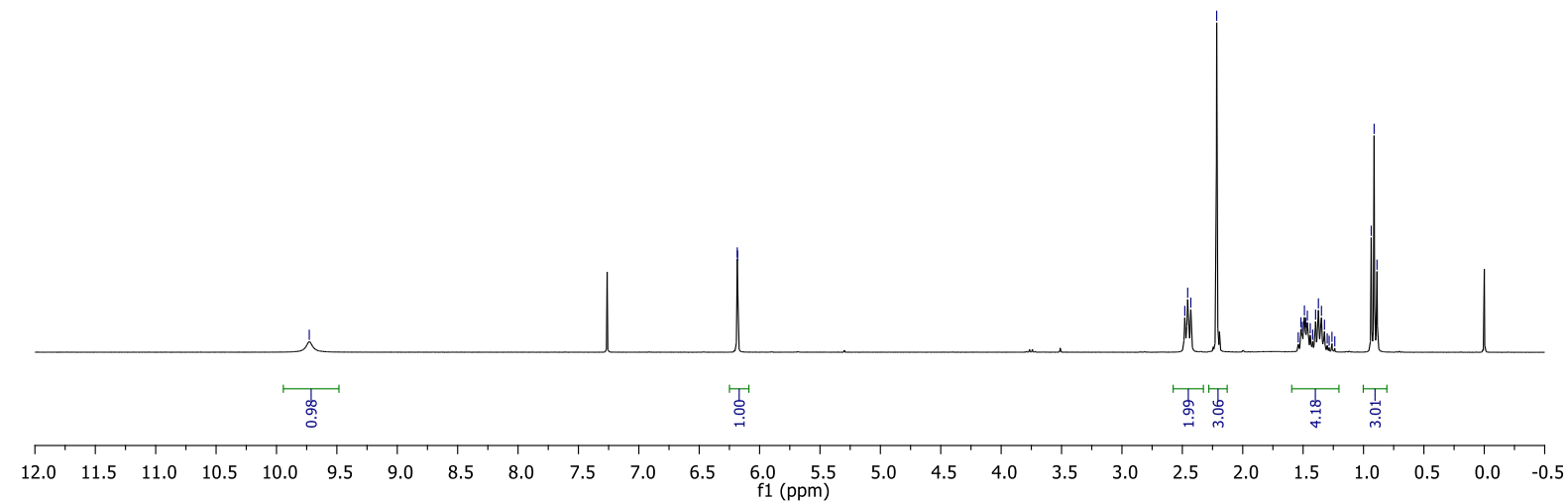

$$
\text { | }
$$

|

|

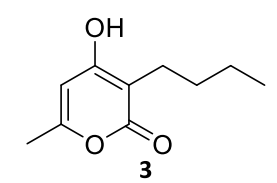

${ }^{13} \mathrm{C}$ NMR (75 MHz, $\mathrm{CDCl}_{3}$ )
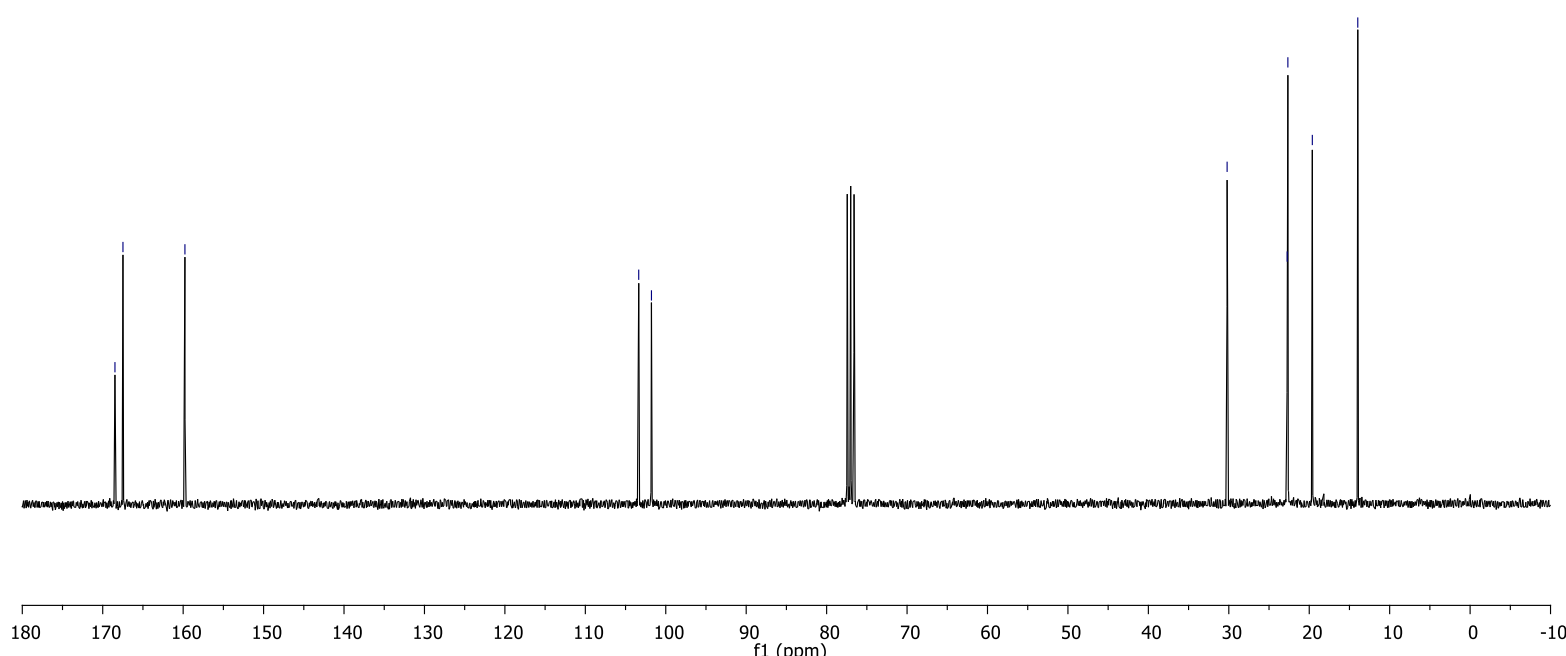

Figure S6: ${ }^{1} \mathrm{H} N M R$ (top) and ${ }^{13} \mathrm{C} N M R$ (bottom) spectra for compound 3. 


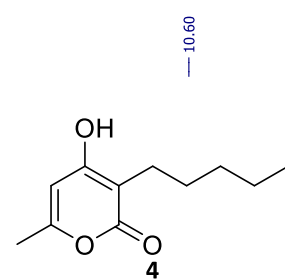

${ }^{1} \mathrm{H} \mathrm{NMR}\left(300 \mathrm{MHz}, \mathrm{CDCl}_{3}\right)$
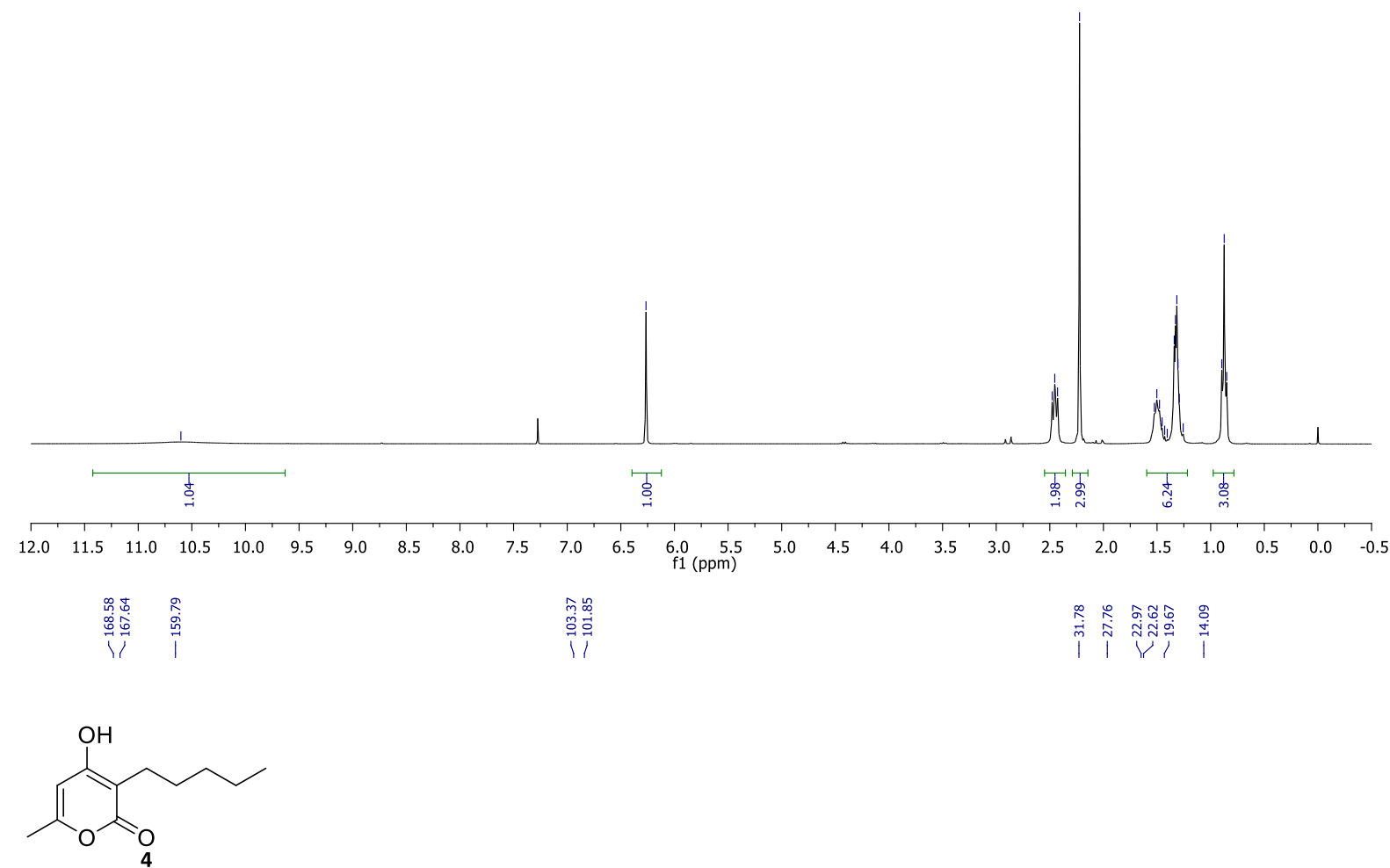

${ }^{13} \mathrm{CNMR}\left(75 \mathrm{MHz}, \mathrm{CDCl}_{3}\right)$

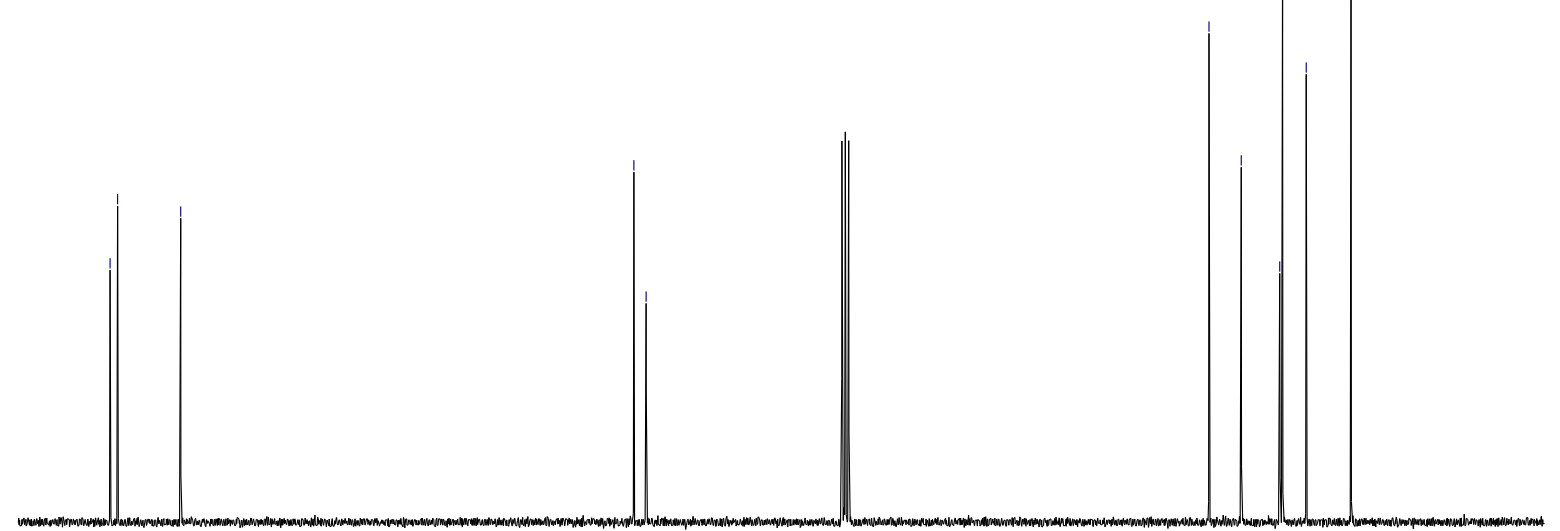

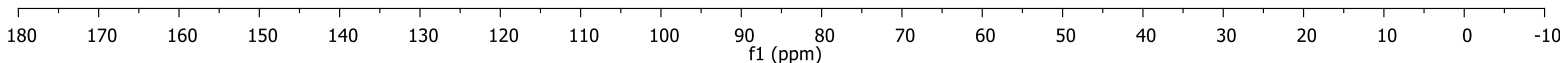

Figure S7: ${ }^{1} \mathrm{H}$ NMR (top) and ${ }^{13} \mathrm{C} N \mathrm{NR}$ (bottom) spectra for compound 4. 

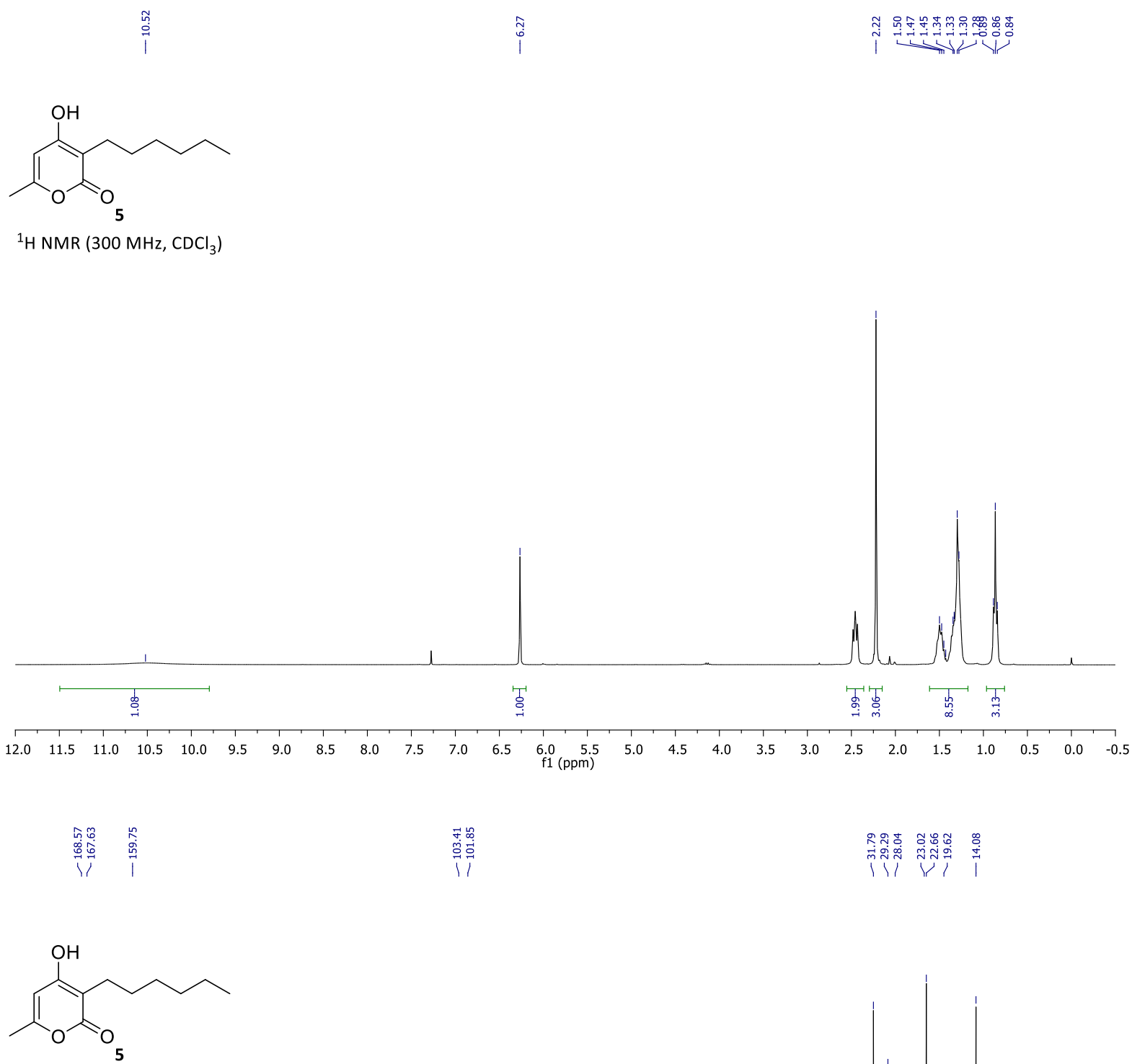

${ }^{13} \mathrm{C} \mathrm{NMR}\left(75 \mathrm{MHz}, \mathrm{CDCl}_{3}\right)$
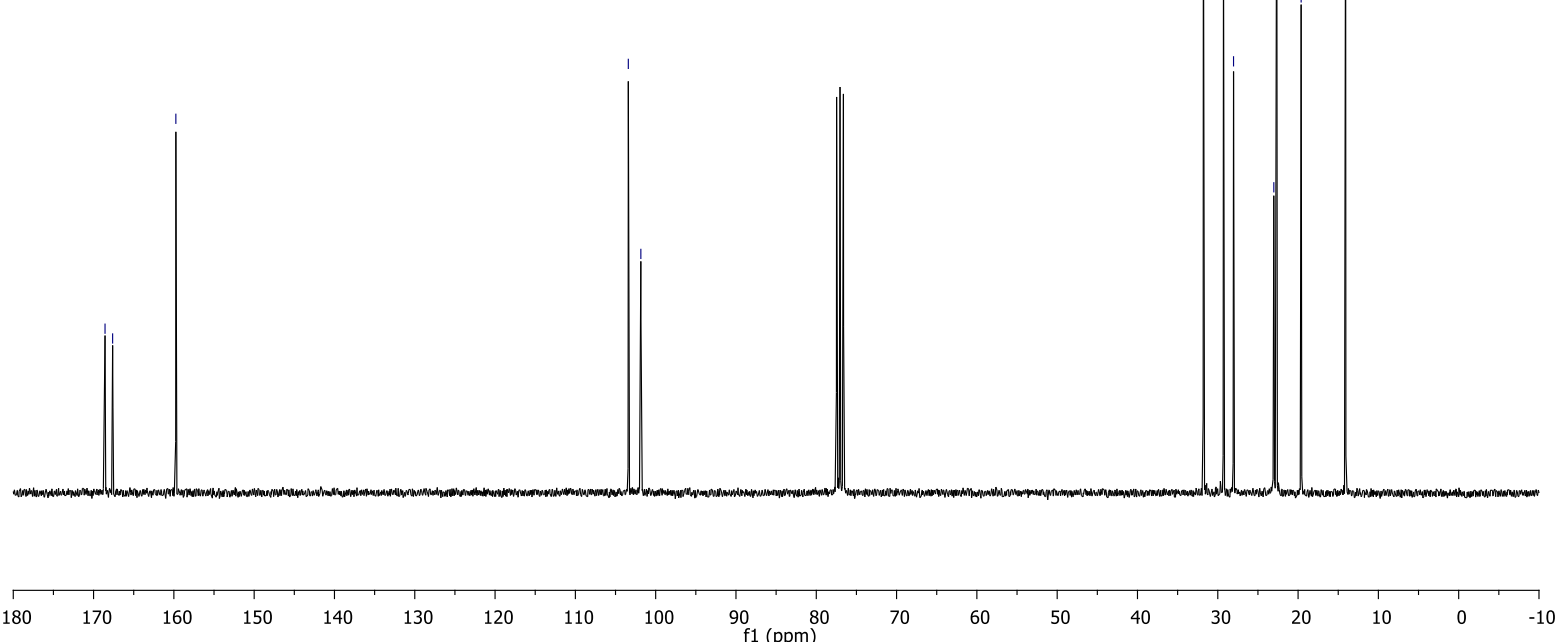

Figure S8: ${ }^{1} \mathrm{H} N M R$ (top) and ${ }^{13} \mathrm{C} N M R$ (bottom) spectra for compound $\mathbf{5}$. 


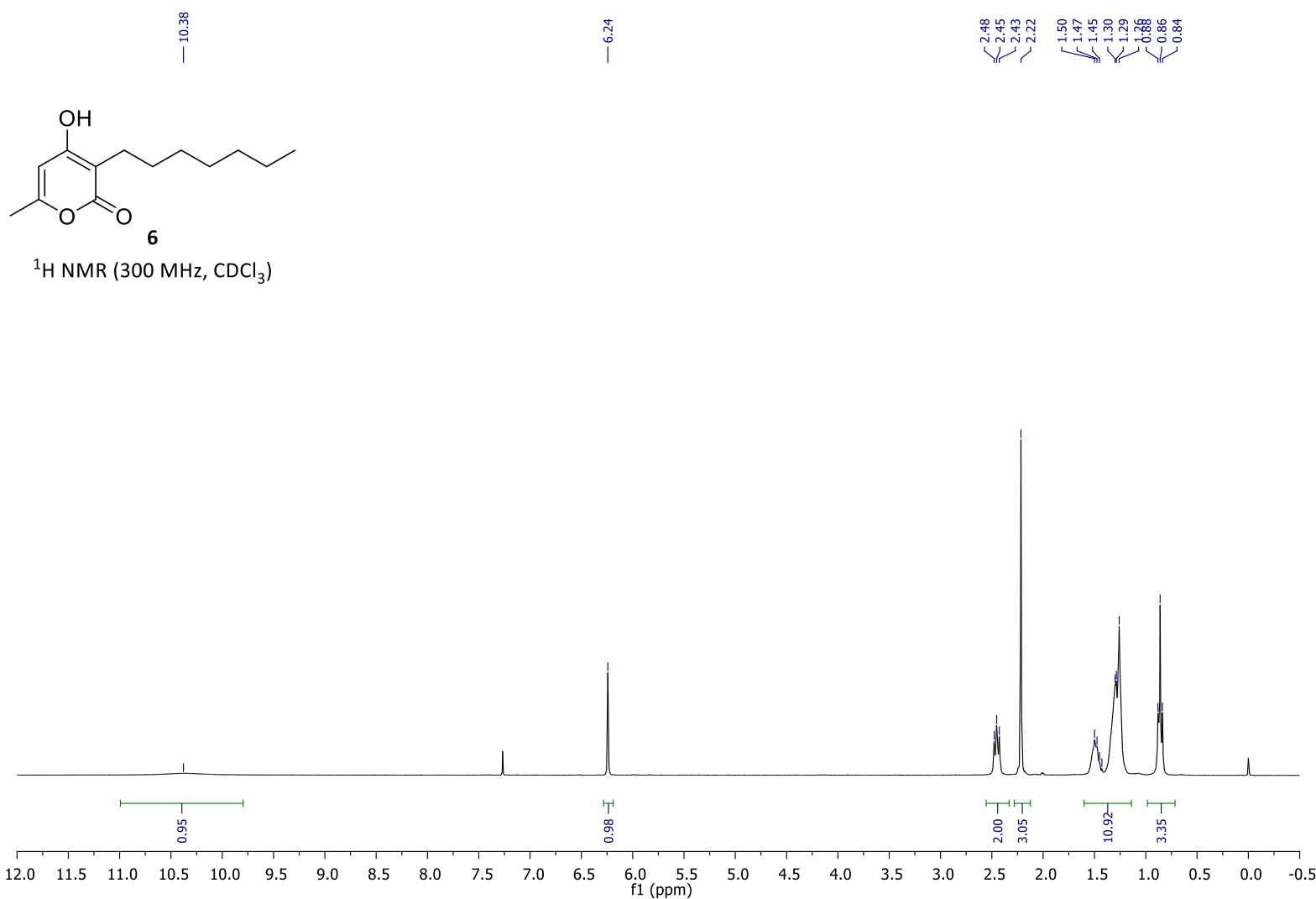

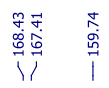

11

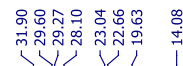

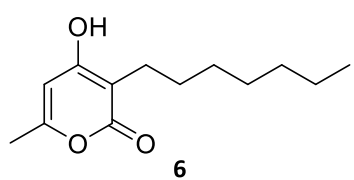

${ }^{13} \mathrm{C}$ NMR (75 MHz, $\mathrm{CDCl}_{3}$ )
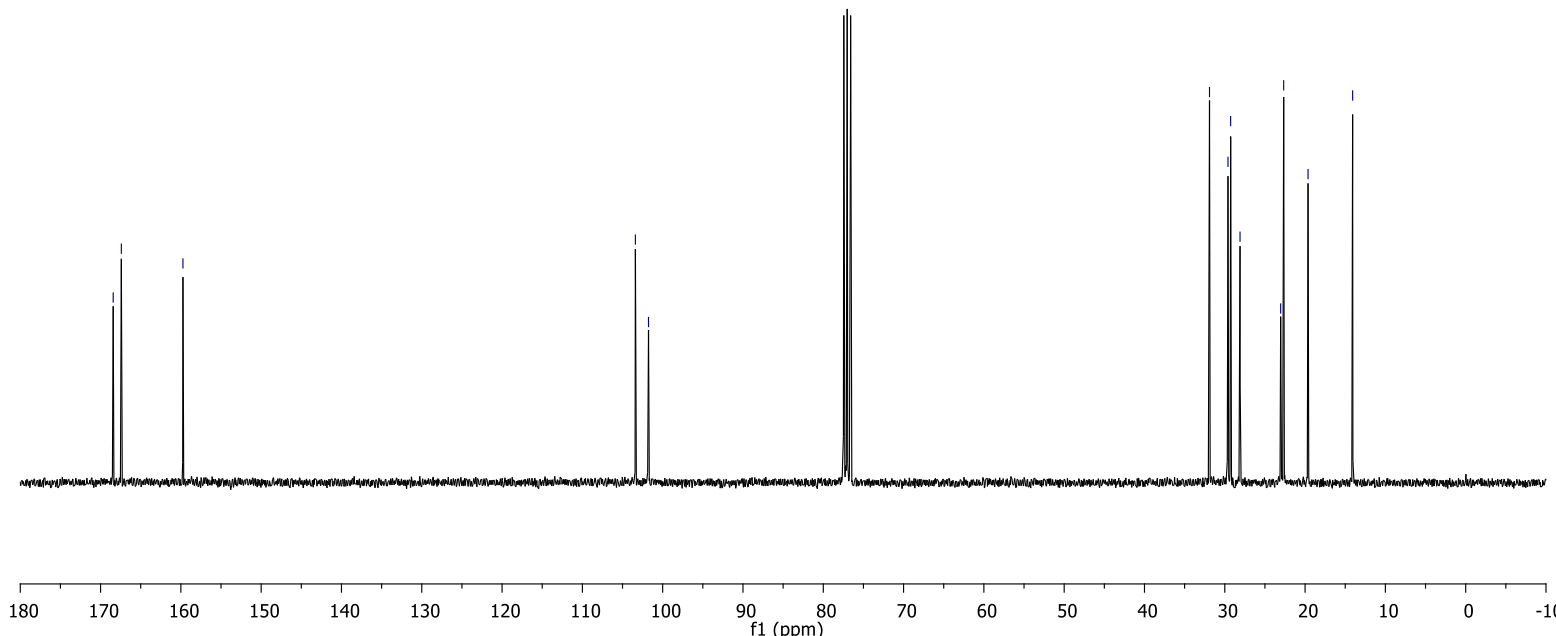

Figure S9: ${ }^{1} H$ NMR (top) and ${ }^{13} \mathrm{C} N M R$ (bottom) spectra for compound 6 . 
<smiles>CCCCCCCCc1c(O)cc(C)oc1=O</smiles>

${ }^{1} \mathrm{H} \mathrm{NMR}\left(300 \mathrm{MHz}, \mathrm{CDCl}_{3}\right)$

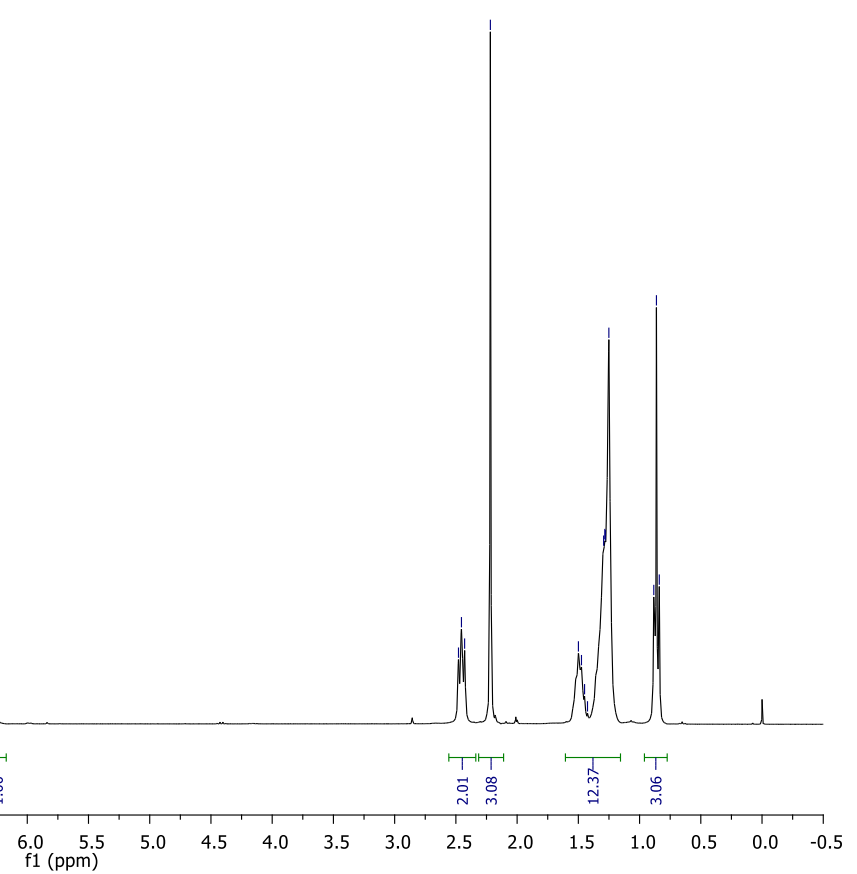

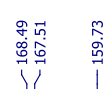

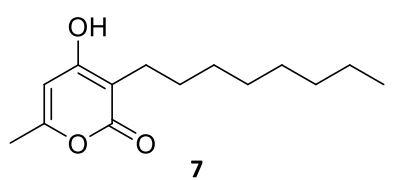

${ }^{13} \mathrm{C}$ NMR $\left(75 \mathrm{MHz}, \mathrm{CDCl}_{3}\right)$

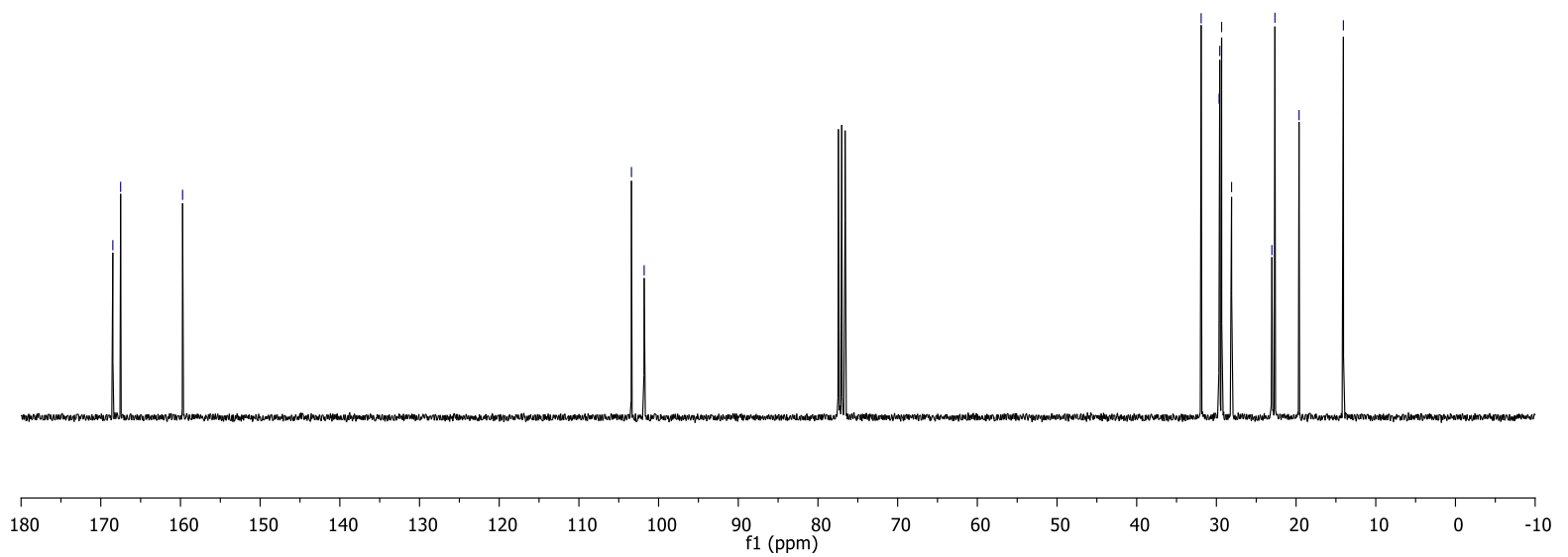

Figure S10: ${ }^{1} \mathrm{H} N \mathrm{NR}$ (top) and ${ }^{13} \mathrm{C} N \mathrm{NM}$ (bottom) spectra for compound 7. 


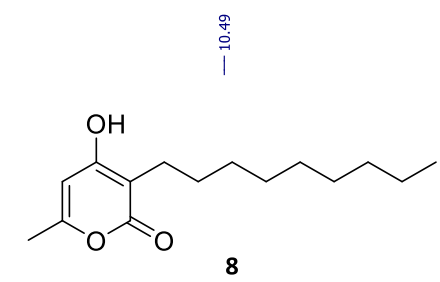

${ }^{1} \mathrm{H}$ NMR $\left(300 \mathrm{MHz}, \mathrm{CDCl}_{3}\right)$

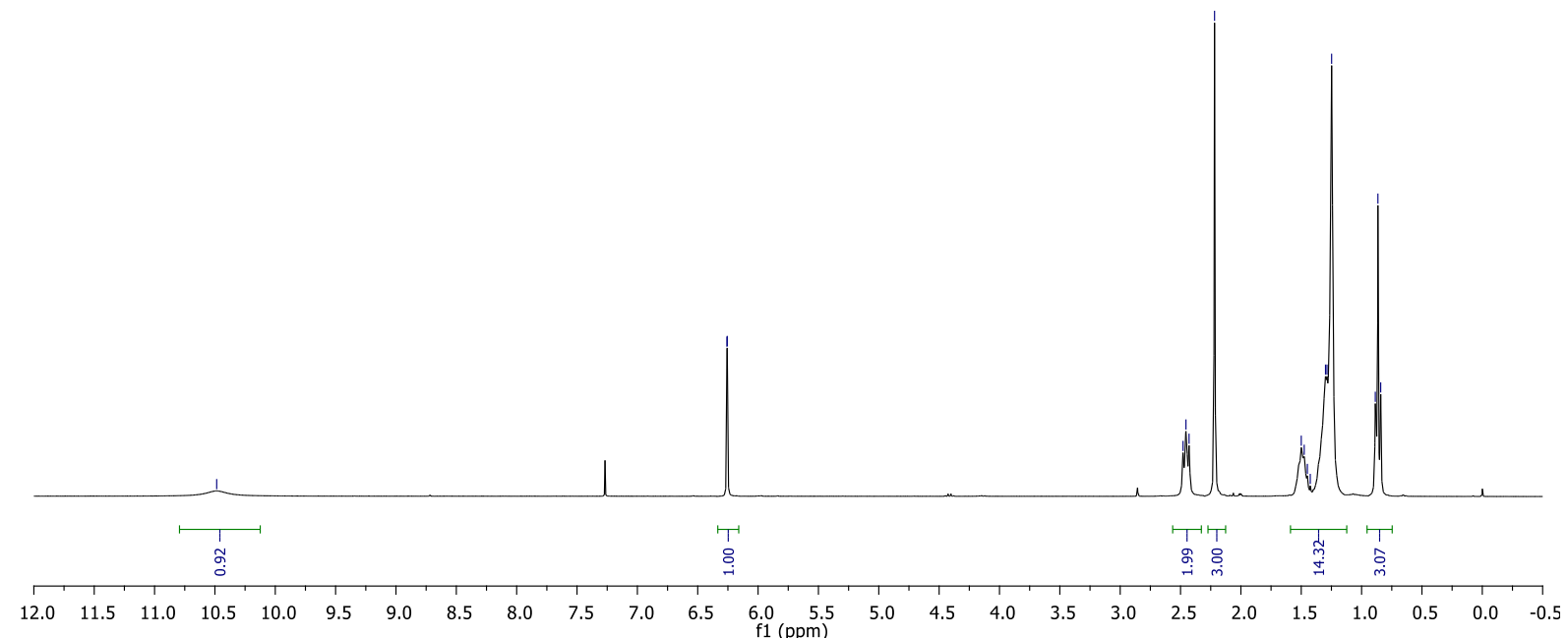

员依

|

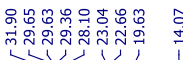

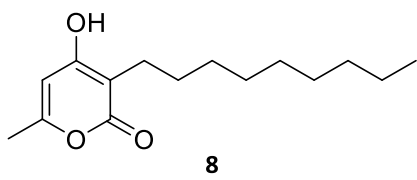

${ }^{13} \mathrm{C}$ NMR $\left(75 \mathrm{MHz}, \mathrm{CDCl}_{3}\right)$

Nit

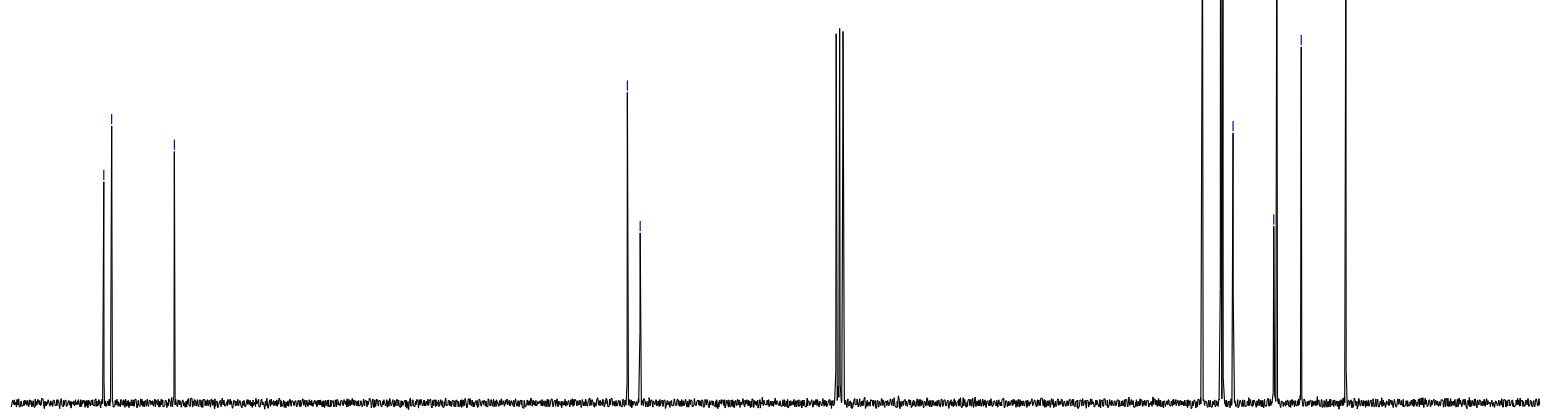

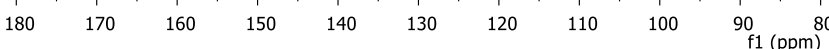

Figure S11: ${ }^{1} \mathrm{H} N \mathrm{NR}$ (top) and ${ }^{13} \mathrm{C} N \mathrm{NR}$ (bottom) spectra for compound 8 . 

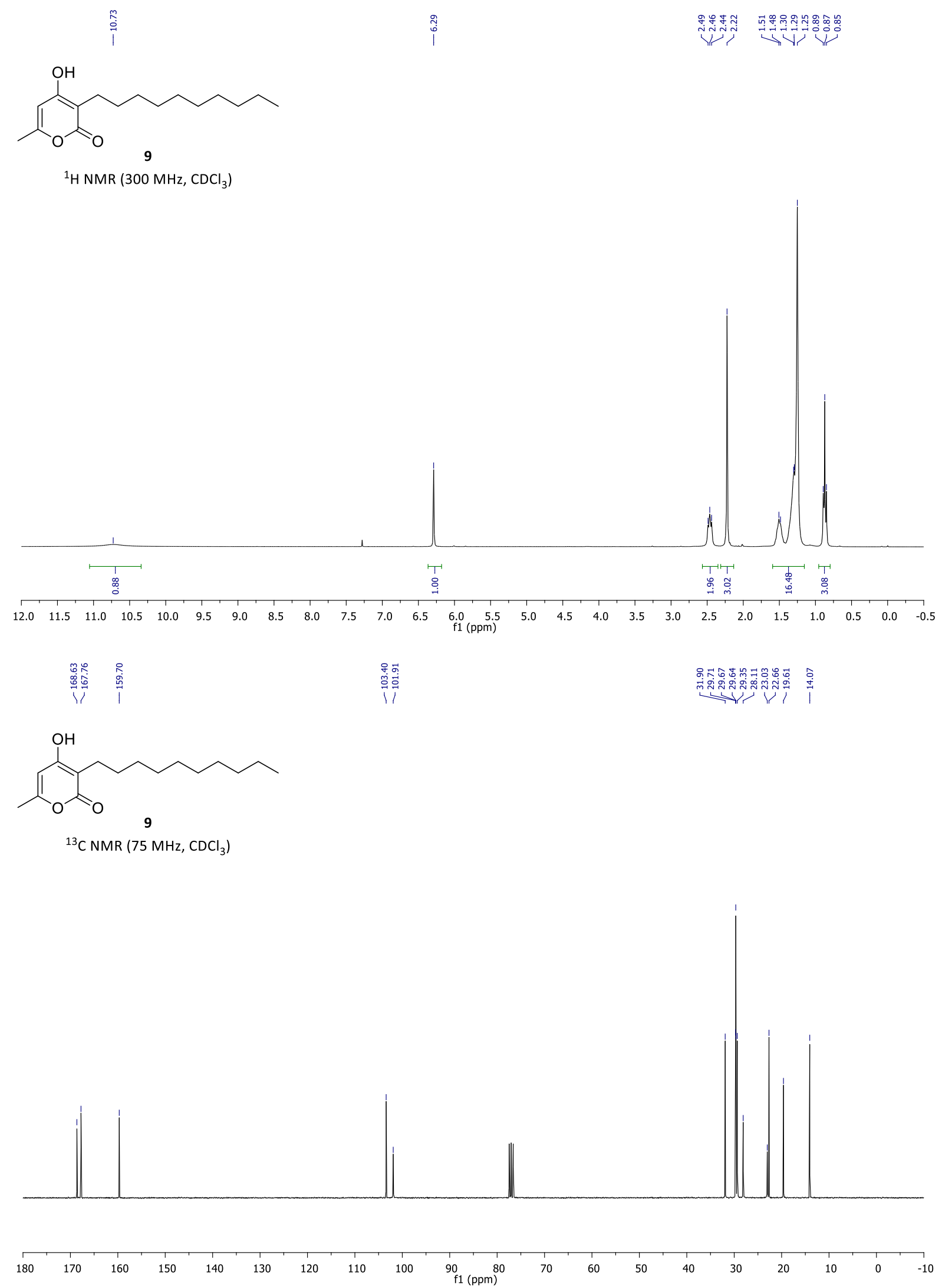

Figure S12: ${ }^{1} H$ NMR (top) and ${ }^{13}$ C NMR (bottom) spectra for compound 9. 


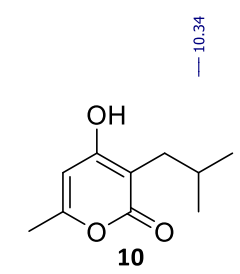

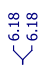

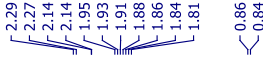

${ }^{1} \mathrm{H}$ NMR $\left(300 \mathrm{MHz}, \mathrm{CDCl}_{3}\right)$

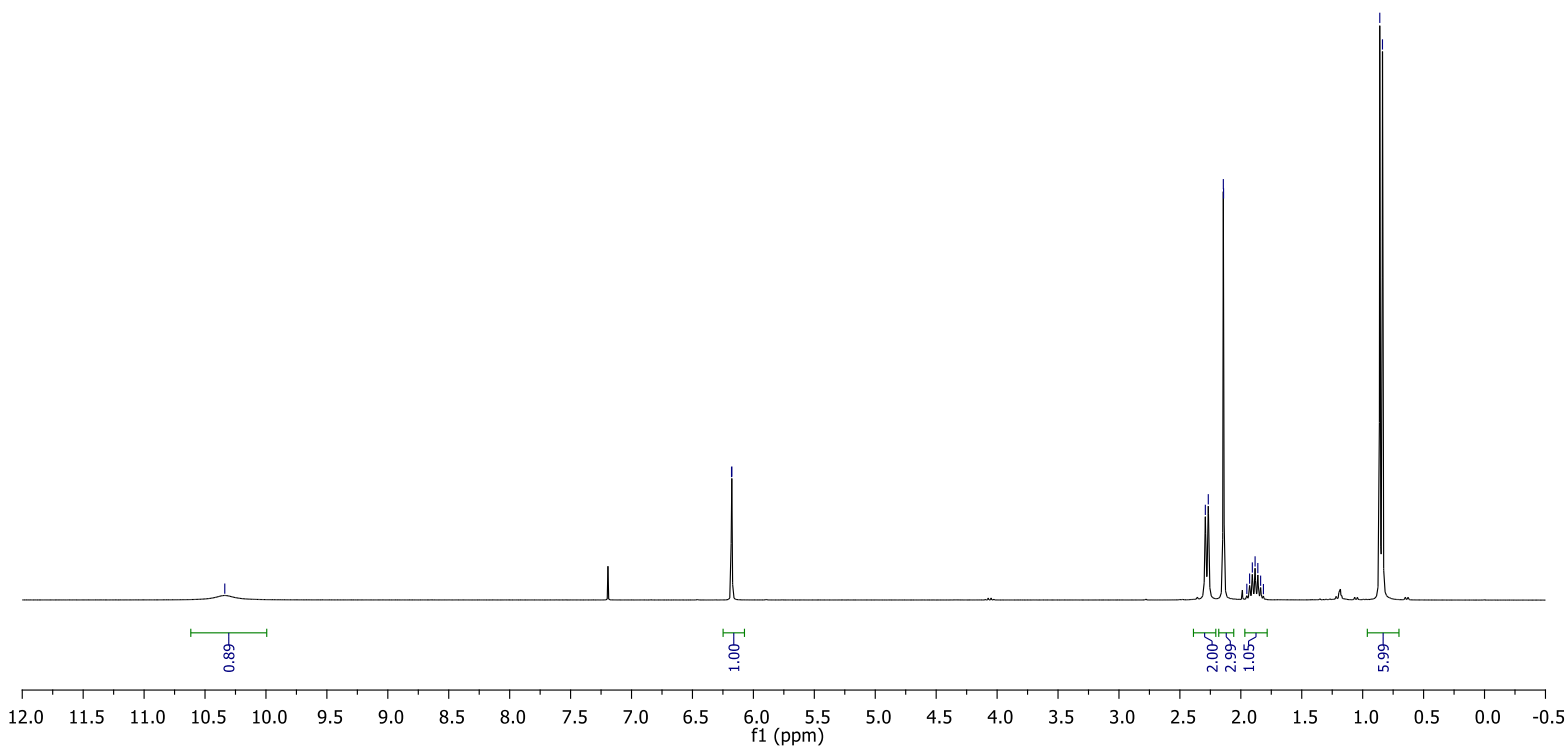

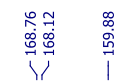

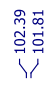

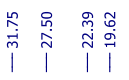

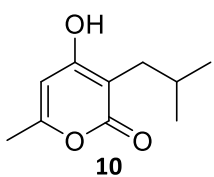

${ }^{13} \mathrm{CNMR}\left(75 \mathrm{MHz}, \mathrm{CDCl}_{3}\right)$

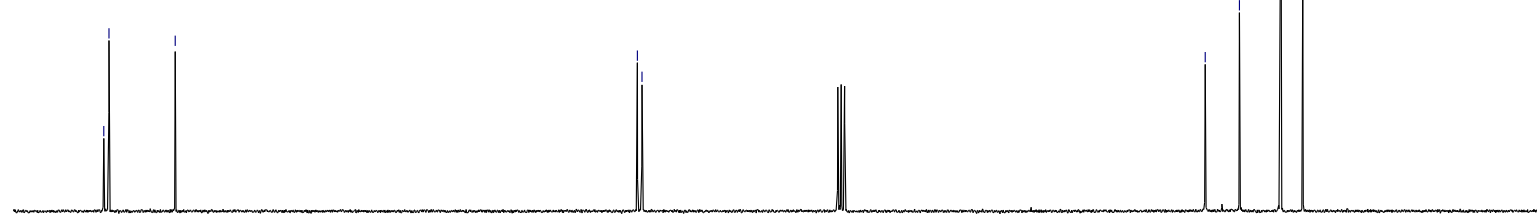

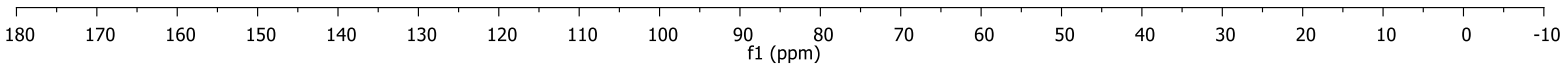

Figure S13: ${ }^{1} \mathrm{H} N \mathrm{NR}$ (top) and ${ }^{13} \mathrm{C}$ NMR (bottom) spectra for compound 10. 


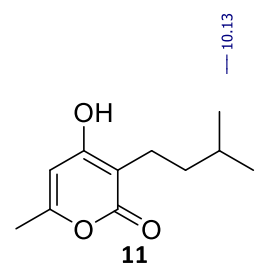

${ }^{1} \mathrm{H}$ NMR $\left(300 \mathrm{MHz}, \mathrm{CDCl}_{3}\right.$ )

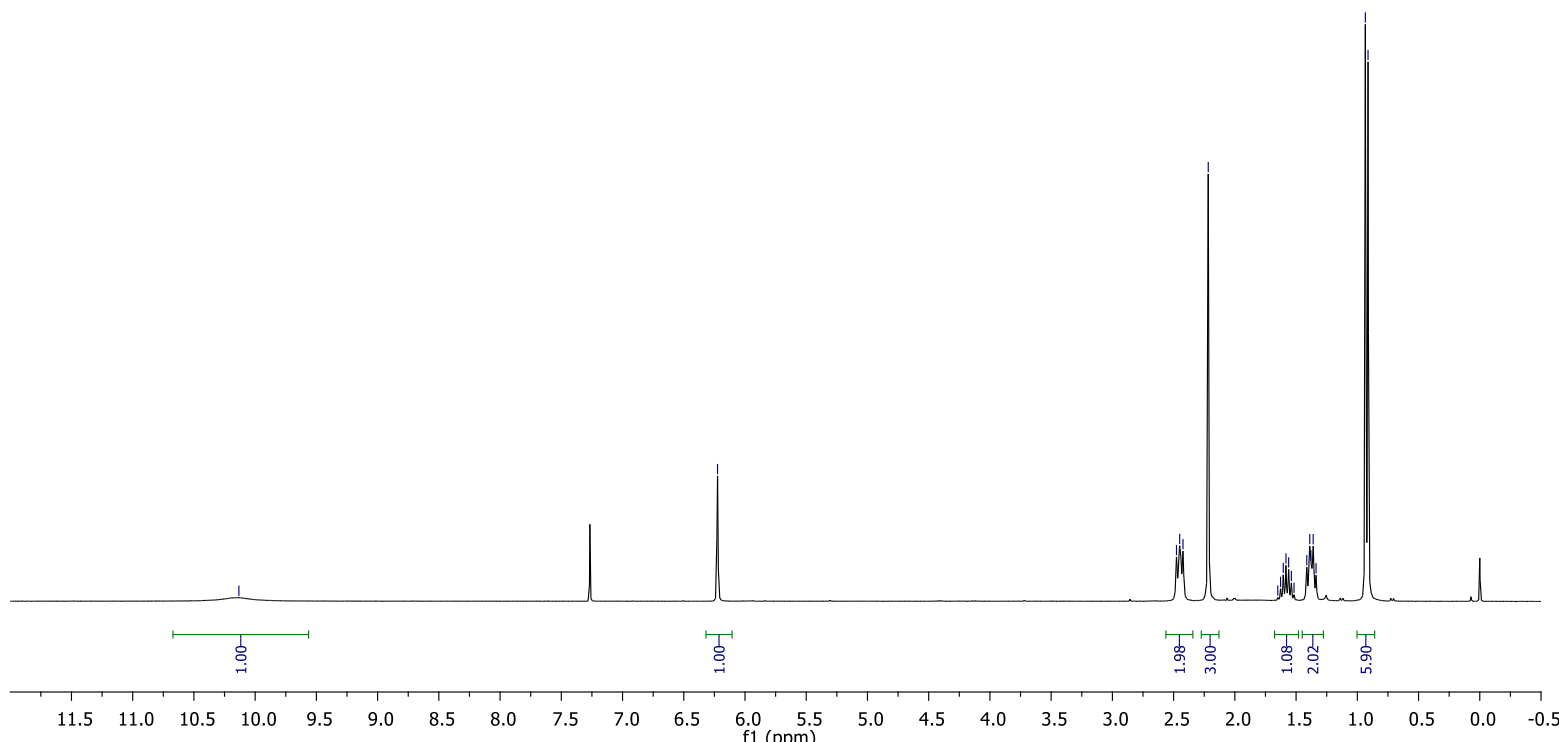

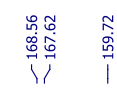

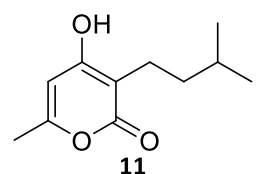

${ }^{13} \mathrm{C}$ NMR $\left(75 \mathrm{MHz}, \mathrm{CDCl}_{3}\right)$
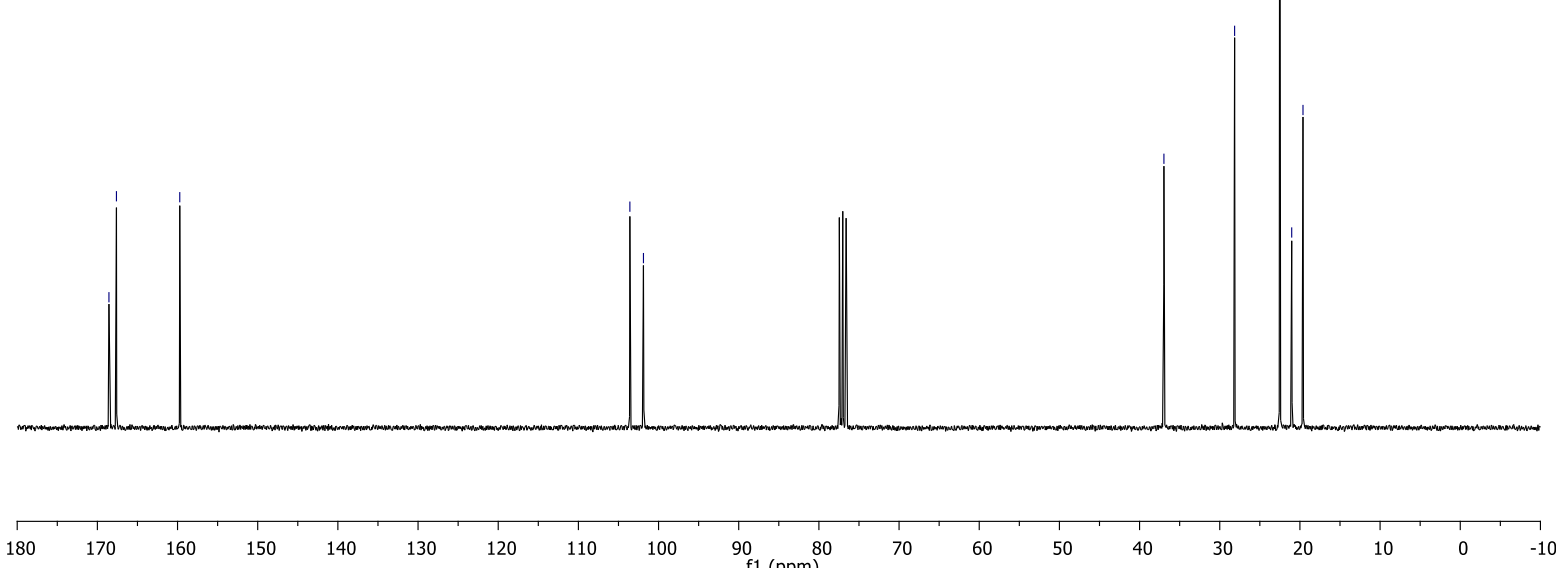

Figure S14: ${ }^{1} \mathrm{H} N \mathrm{NMR}$ (top) and ${ }^{13} \mathrm{C} N M R$ (bottom) spectra for compound 11. 
<smiles>Cc1cc(O)c(Cc2ccccc2)c(=O)o1</smiles>

${ }^{1} \mathrm{H} \mathrm{NMR}\left(300 \mathrm{MHz}, \mathrm{CD}_{3} \mathrm{OD}\right)$

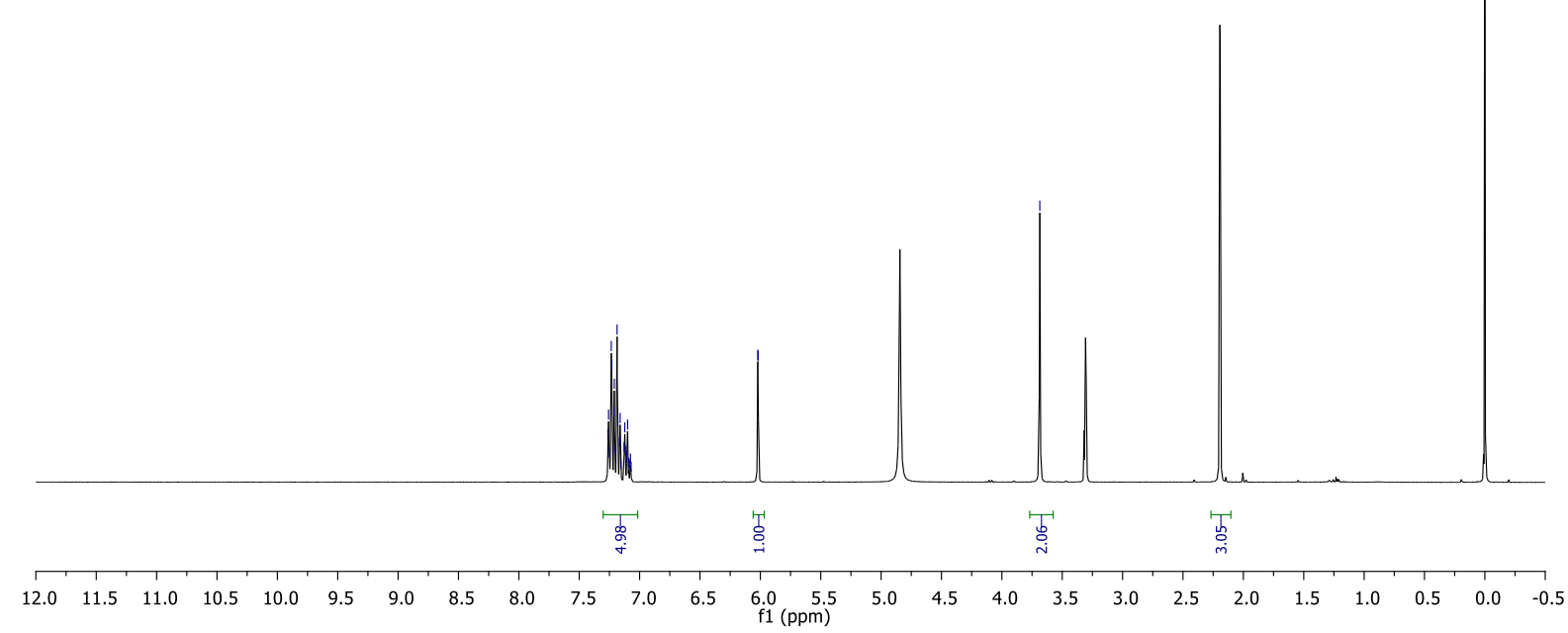<smiles>Cc1cc(O)c(Cc2ccccc2)c(=O)o1</smiles>

${ }^{13} \mathrm{C}$ NMR $\left(75 \mathrm{MHz}, \mathrm{CD}_{3} \mathrm{OD}\right)$
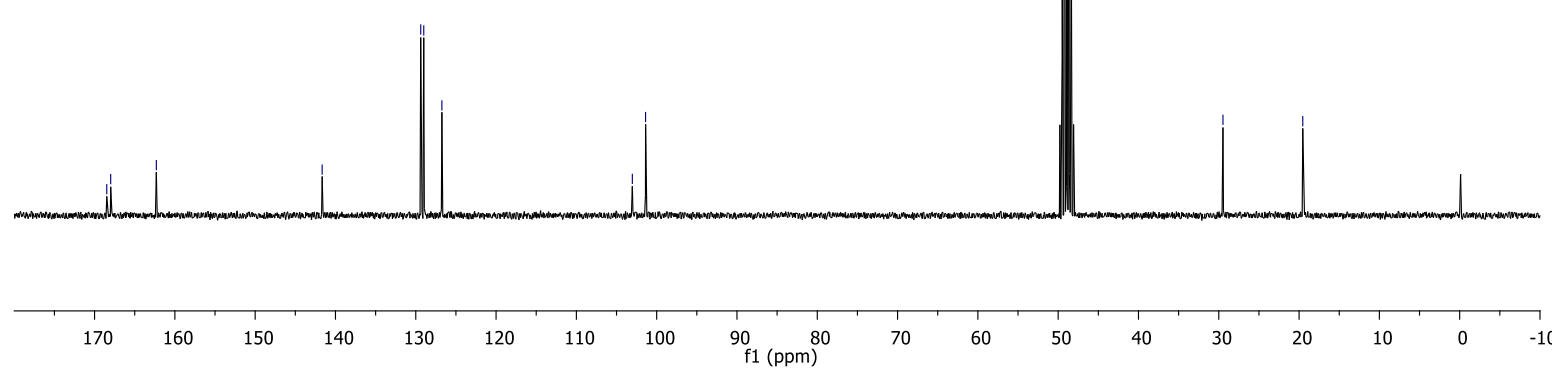

Figure S15: ${ }^{1} \mathrm{H} N \mathrm{NM}$ (top) and ${ }^{13} \mathrm{C} N M R$ (bottom) spectra for compound 12. 


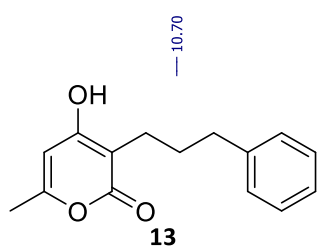

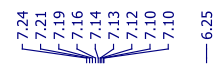

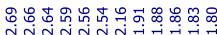

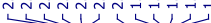

${ }^{1} \mathrm{HNMR}\left(300 \mathrm{MHz}, \mathrm{CDCl}_{3}\right)$

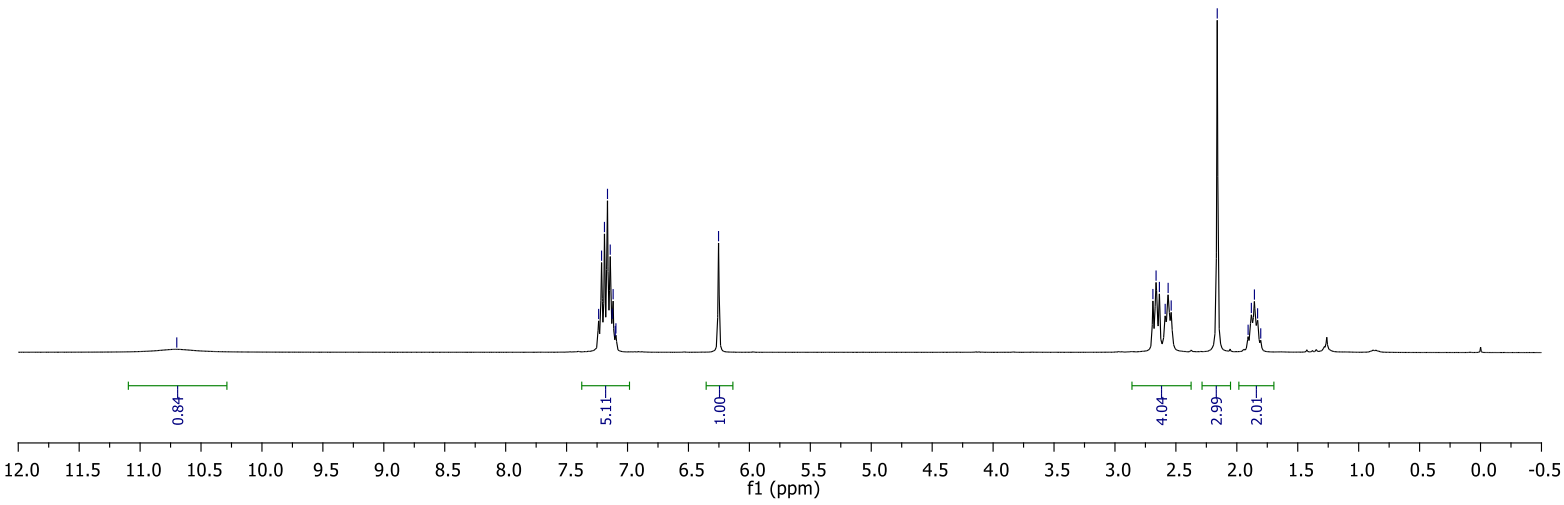

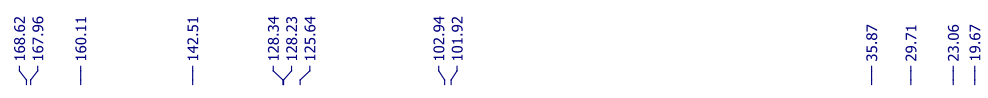<smiles>Cc1cc(O)c(CCCc2ccccc2)c(=O)o1</smiles>

${ }^{13} \mathrm{CNMR}\left(75 \mathrm{MHz}, \mathrm{CDCl}_{3}\right)$

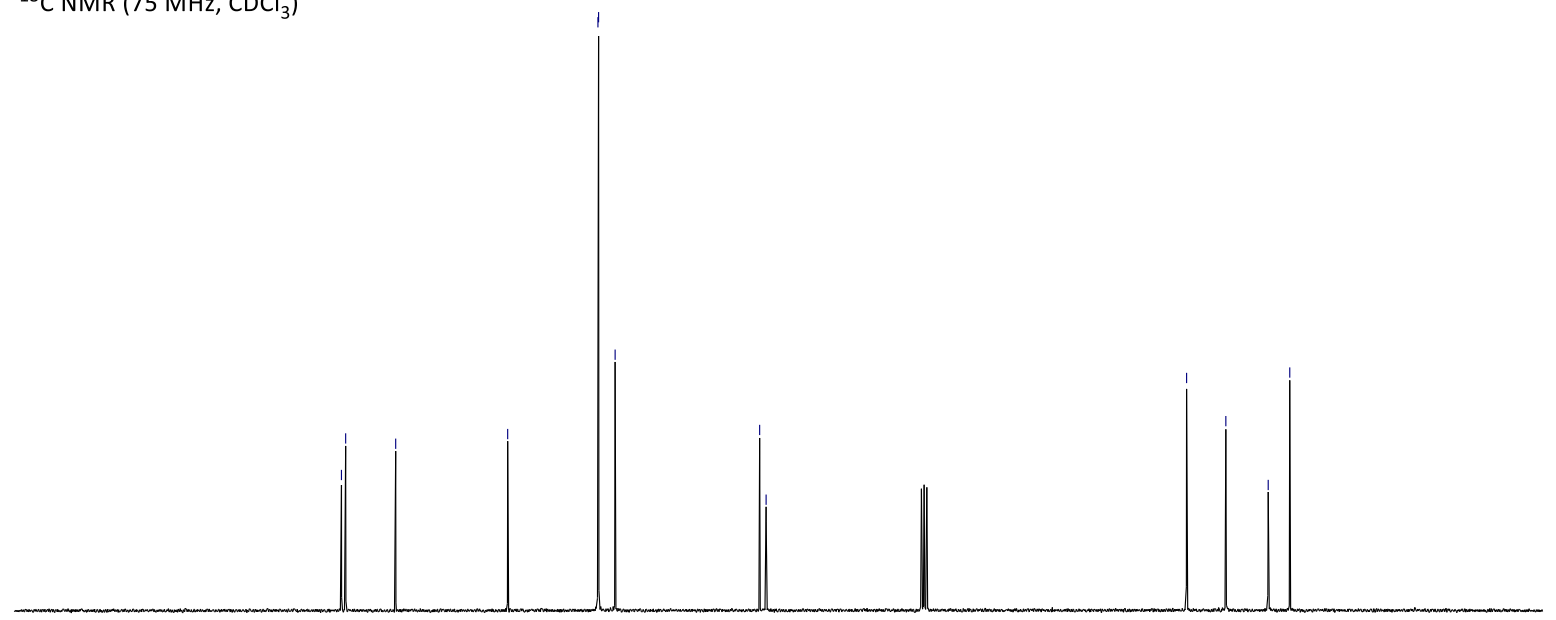

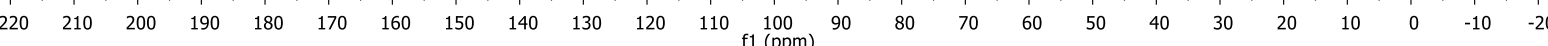

Figure S16: ${ }^{1} \mathrm{H} N \mathrm{NR}$ (top) and ${ }^{13} \mathrm{C}$ NMR (bottom) spectra for compound 13. 


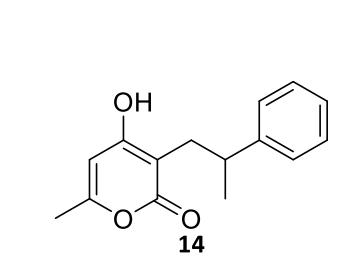

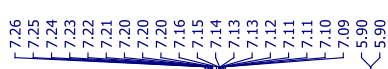

${ }^{1} \mathrm{H} \mathrm{NMR}\left(300 \mathrm{MHz}, \mathrm{CD}_{3} \mathrm{OD} / \mathrm{CDCl}_{3}\right)$

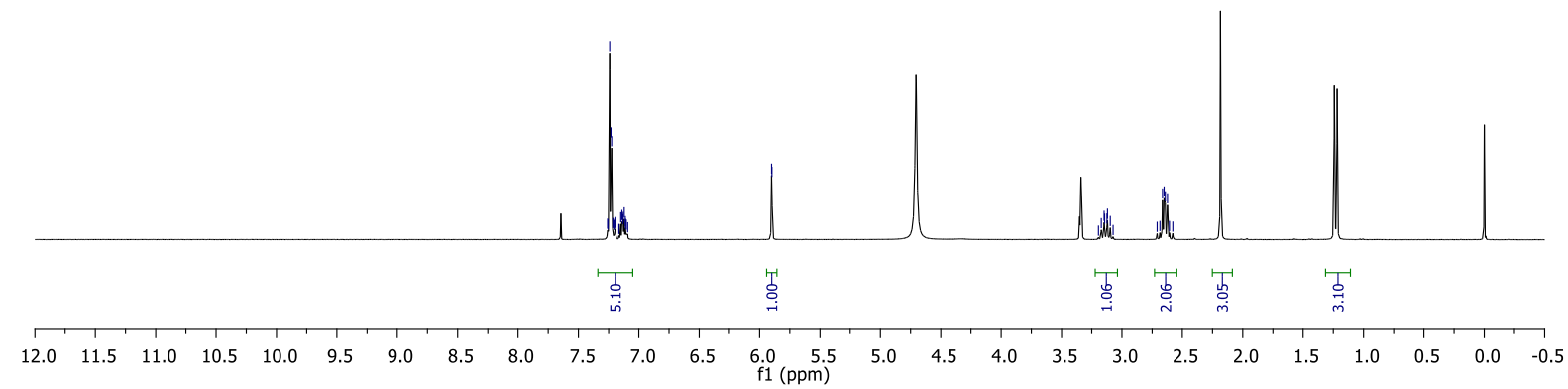

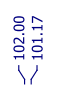<smiles>Cc1cc(O)c(CC(C)c2ccccc2)c(=O)o1</smiles>

${ }^{13} \mathrm{C} \mathrm{NMR}\left(75 \mathrm{MHz}, \mathrm{CD}_{3} \mathrm{OD} / \mathrm{CDCl}_{3}\right)$

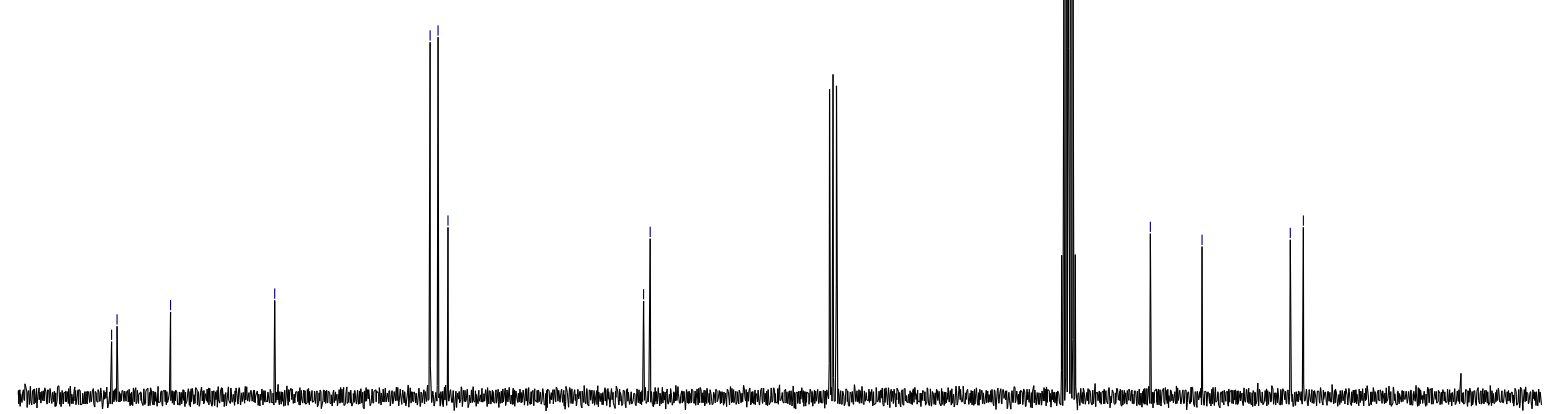

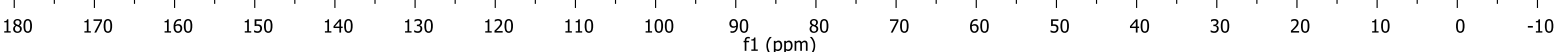

Figure S17: ${ }^{1} H$ NMR (top) and ${ }^{13} C N M R$ (bottom) spectra for compound 14. 


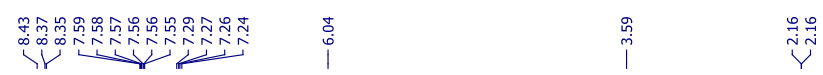<smiles>Cc1cc(O)c(Cc2cccnc2)c(=O)o1</smiles>

${ }^{1} \mathrm{H}$ NMR $\left(300 \mathrm{MHz},\left(\mathrm{CD}_{3}\right)_{2} \mathrm{SO}\right)$
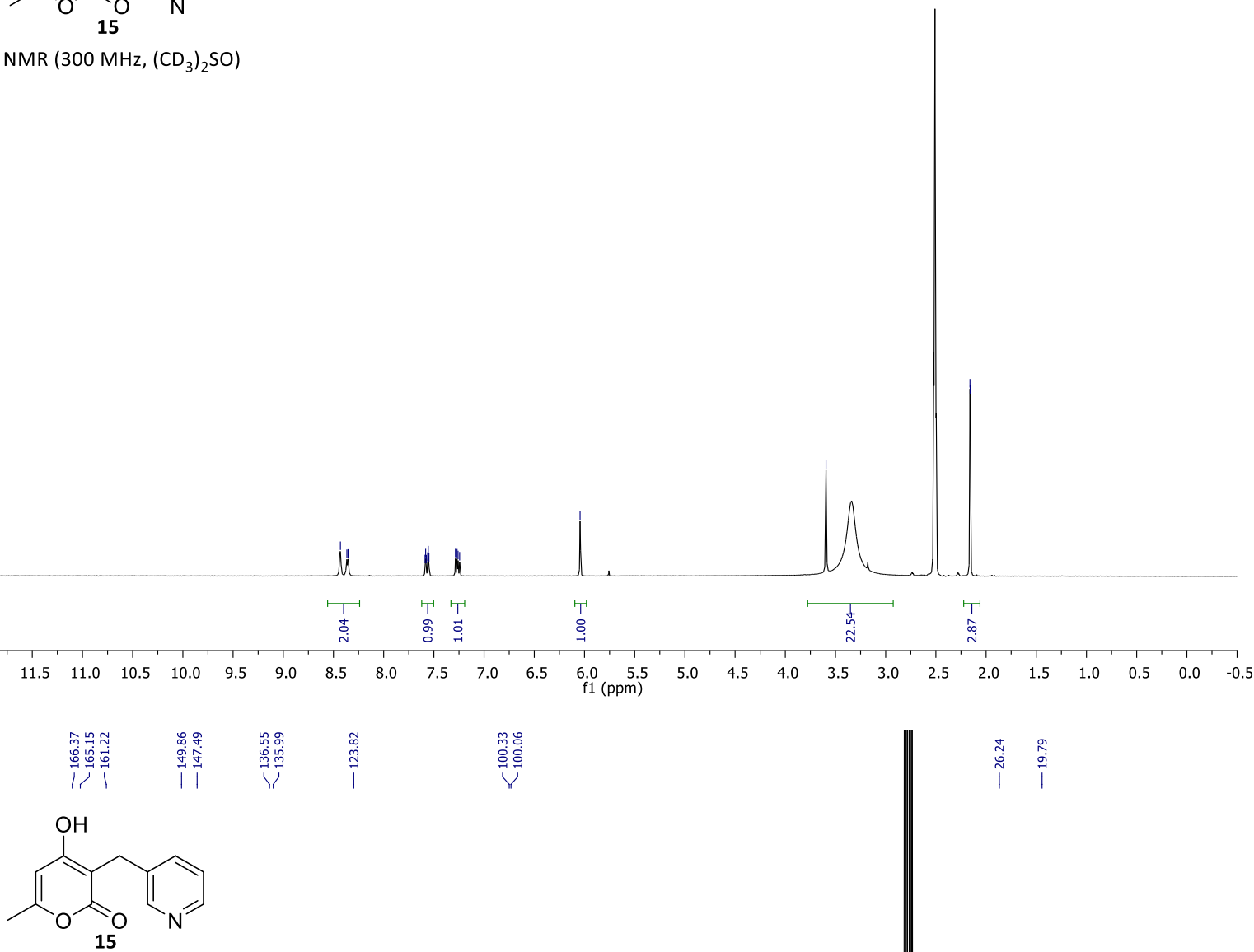

${ }^{13} \mathrm{CNMR}\left(75 \mathrm{MHz},\left(\mathrm{CD}_{3}\right)_{2} \mathrm{SO}\right)$
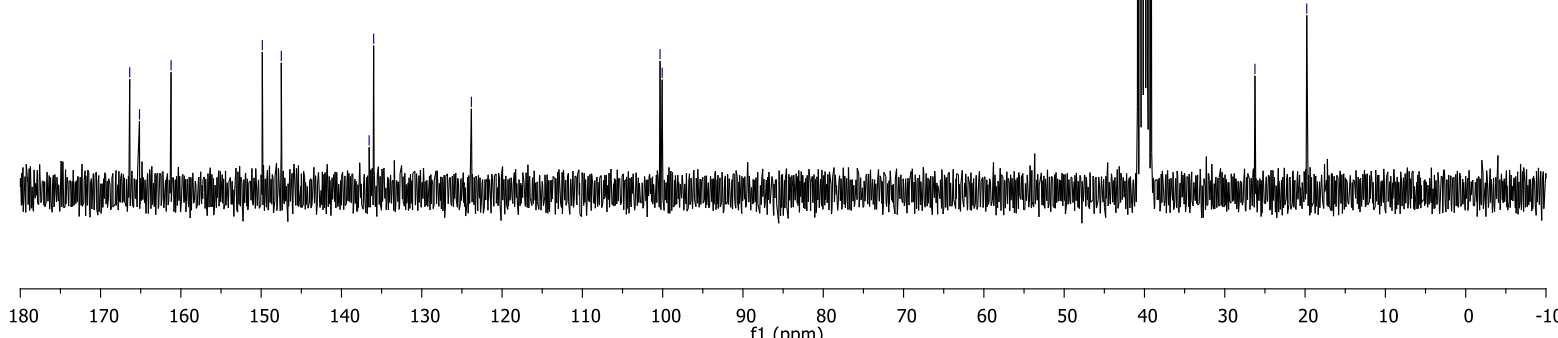

Figure S18: ${ }^{1} \mathrm{H} N \mathrm{NR}$ (top) and ${ }^{13} \mathrm{C}$ NMR (bottom) spectra for compound 15. 


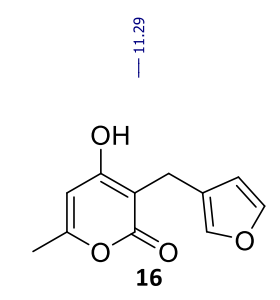

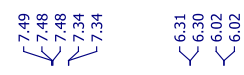

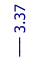

${ }^{1} \mathrm{H}$ NMR (300 MHz, $\left.\left(\mathrm{CD}_{3}\right)_{2} \mathrm{SO}\right)$
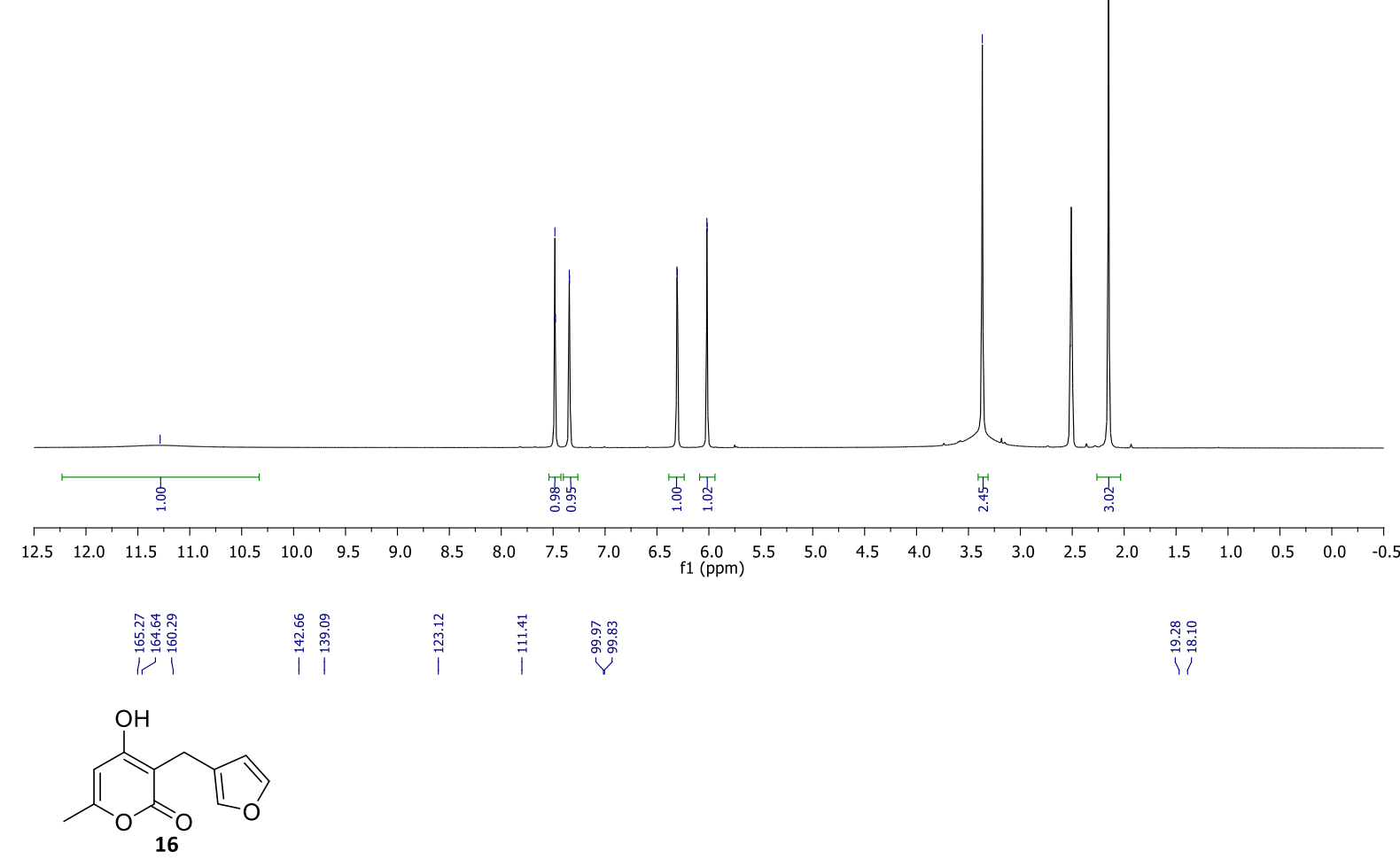

${ }^{13} \mathrm{C} \mathrm{NMR}\left(75 \mathrm{MHz},\left(\mathrm{CD}_{3}\right){ }_{2} \mathrm{SO}\right)$

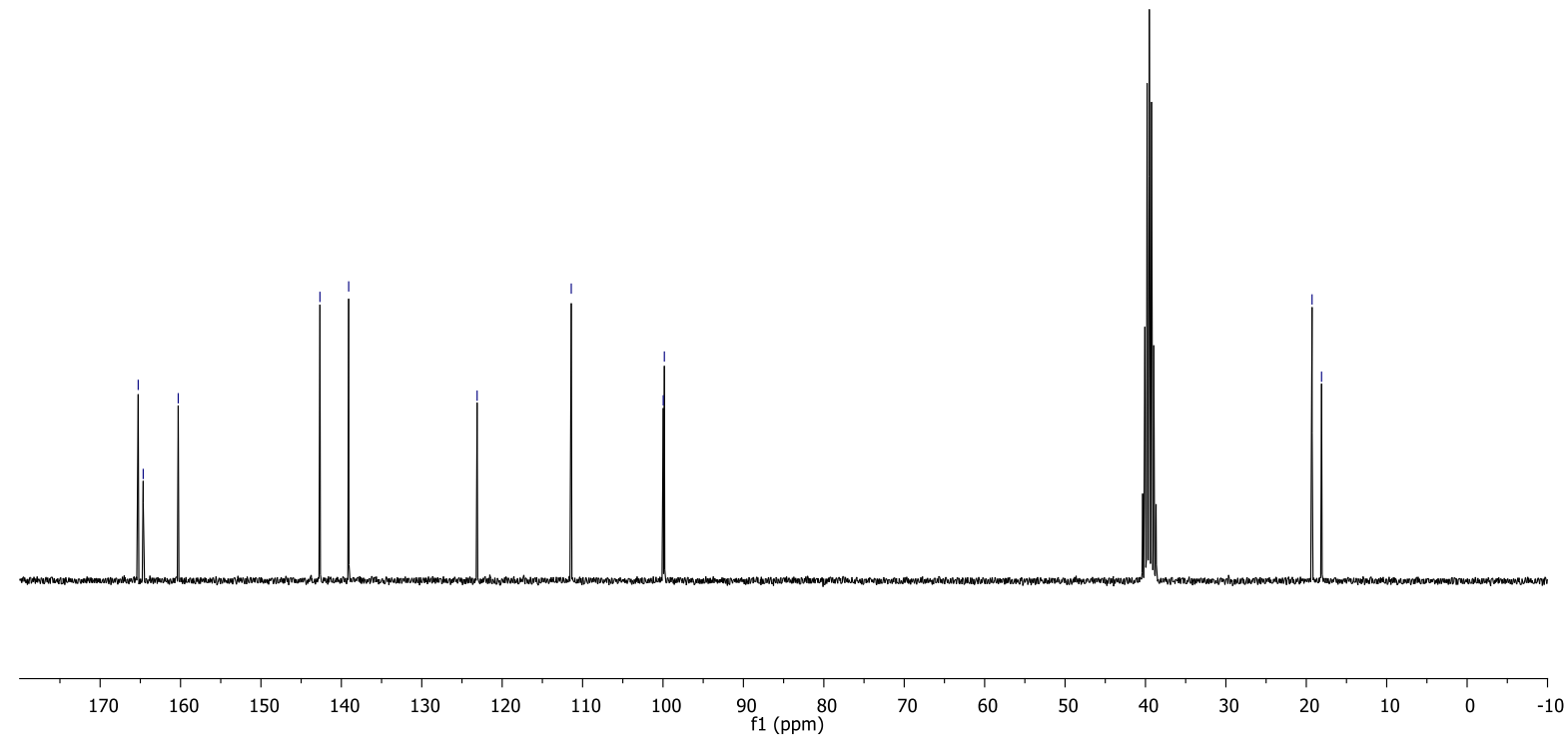

Figure S19: ${ }^{1} \mathrm{H} N M R$ (top) and ${ }^{13} \mathrm{C} N M R$ (bottom) spectra for compound 16. 


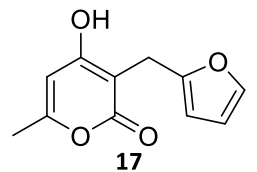

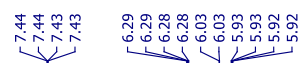

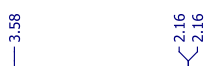

${ }^{1} \mathrm{H}$ NMR $\left(300 \mathrm{MHz},\left(\mathrm{CD}_{3}\right)_{2} \mathrm{SO}\right)$
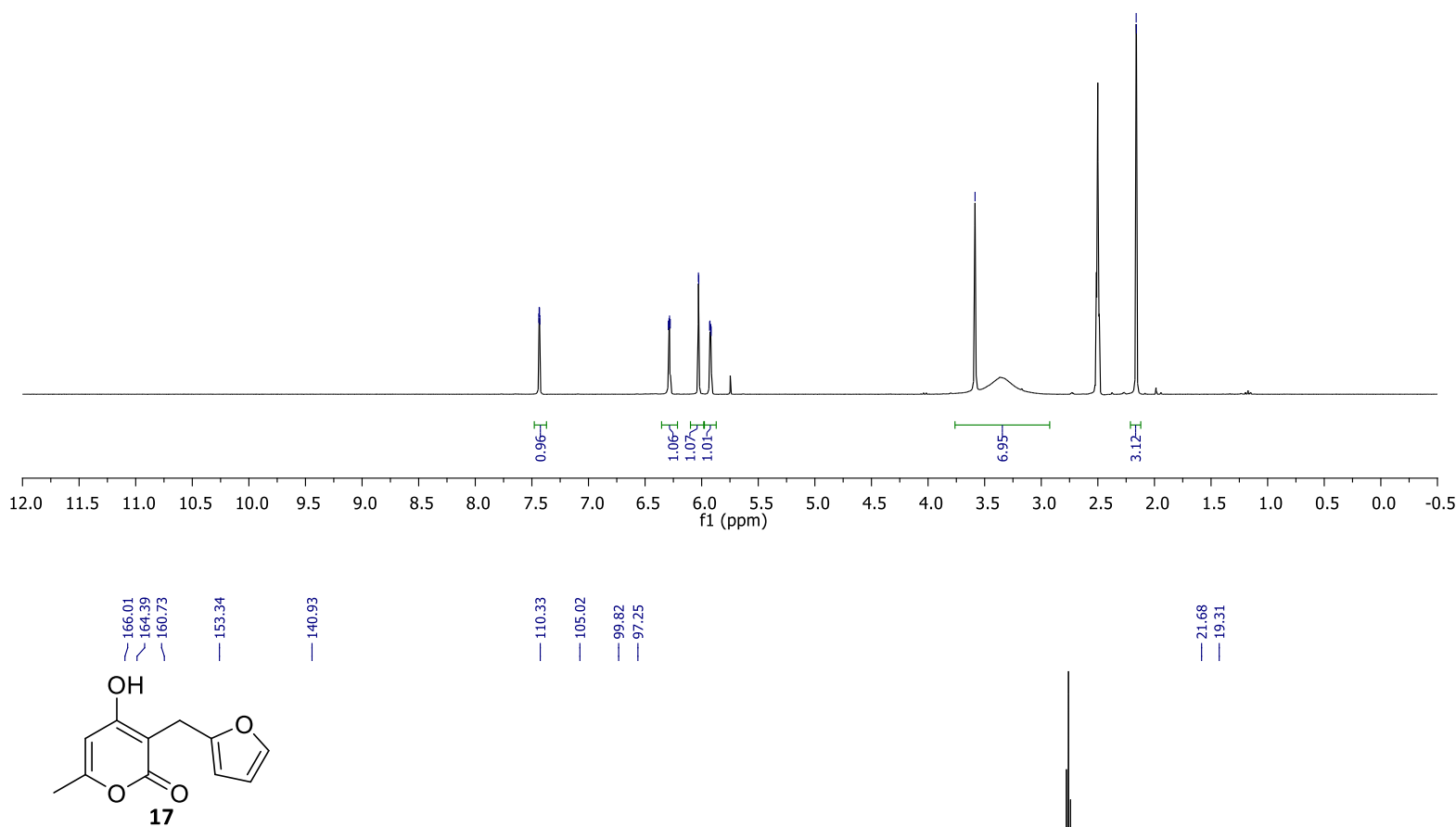

${ }^{13} \mathrm{C}$ NMR $\left(75 \mathrm{MHz},\left(\mathrm{CD}_{3}\right)_{2} \mathrm{SO}\right)$

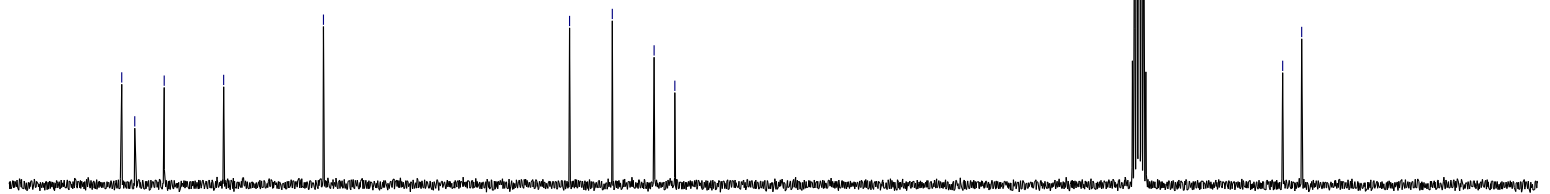

Figure S20: ${ }^{1} \mathrm{H}$ NMR (top) and ${ }^{13} \mathrm{C}$ NMR (bottom) spectra for compound 17. 


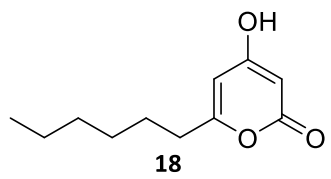

${ }^{1} \mathrm{H}$ NMR (300 MHz, $\mathrm{CDCl}_{3}$ )

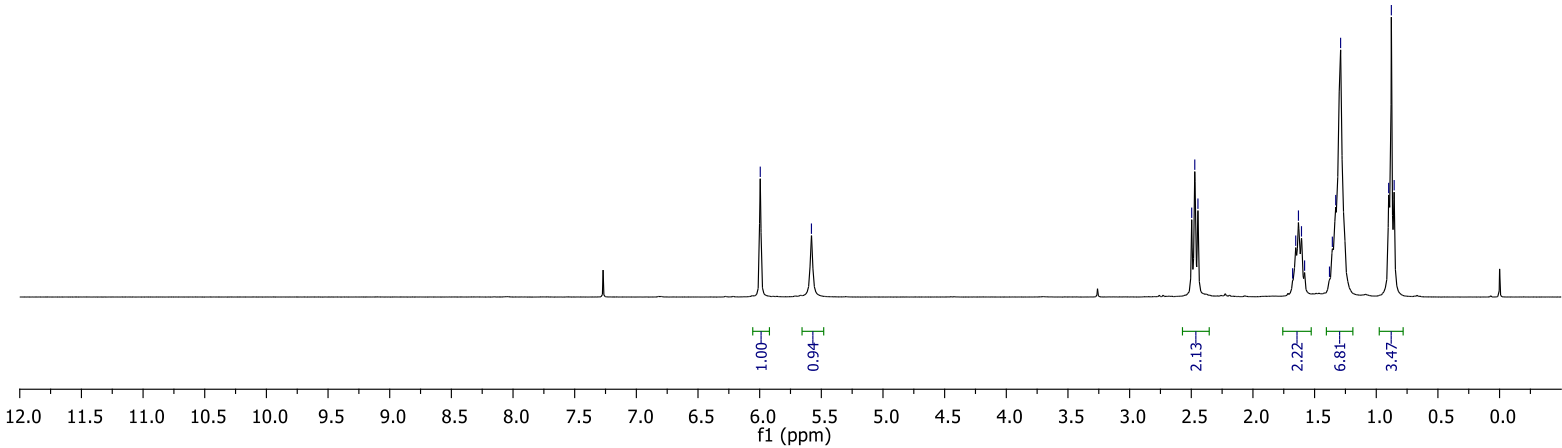

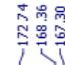

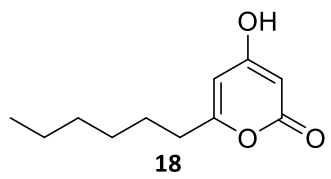

${ }^{13} \mathrm{C}$ NMR $\left(75 \mathrm{MHz}, \mathrm{CDCl}_{3}\right)$
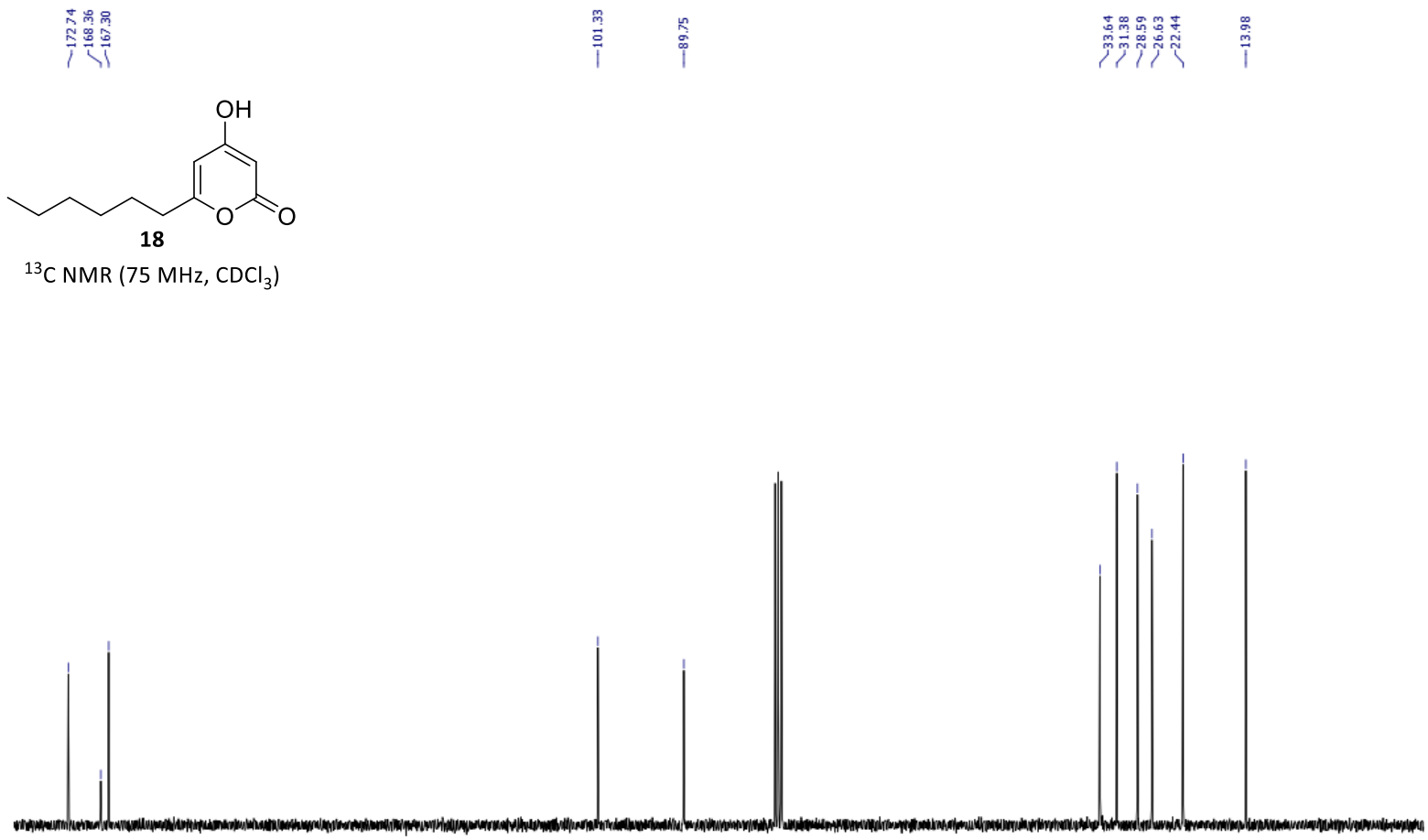

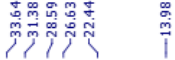

Figure S21: ${ }^{1}$ H NMR (top) and ${ }^{13}$ C NMR (bottom) spectra for compound 18. 


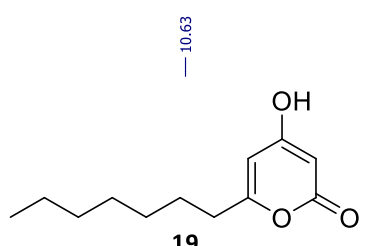

$\stackrel{\overrightarrow{0}}{i} \stackrel{0}{i} \dot{0}$

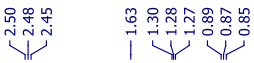

${ }^{1} \mathrm{H}$ NMR $\left(300 \mathrm{MHz}, \mathrm{CDCl}_{3}\right)$

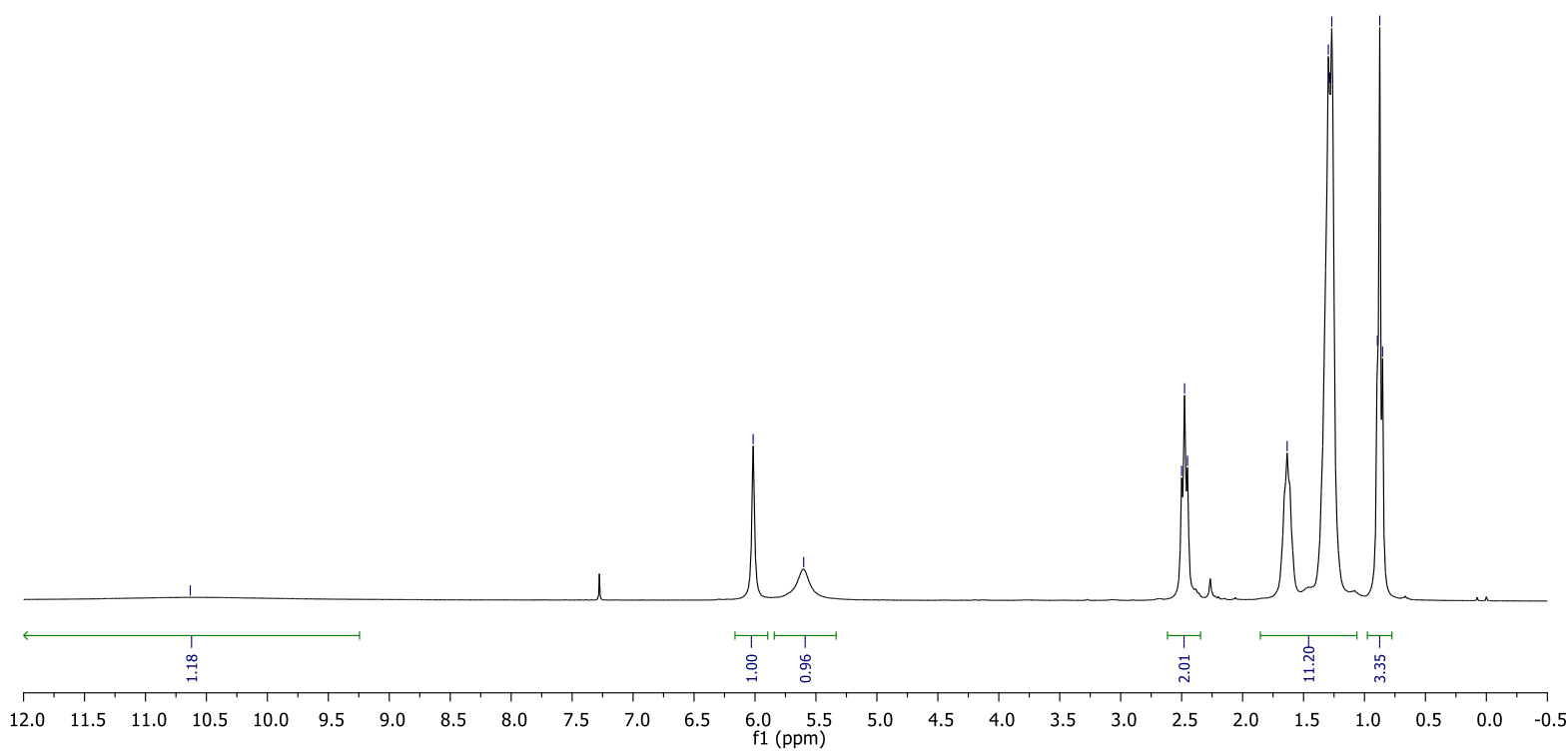

阆

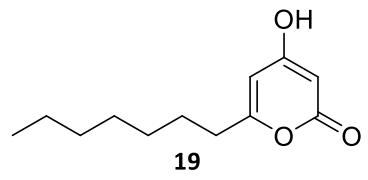

${ }^{13} \mathrm{C} \mathrm{NMR}\left(75 \mathrm{MHz}, \mathrm{CDCl}_{3}\right)$

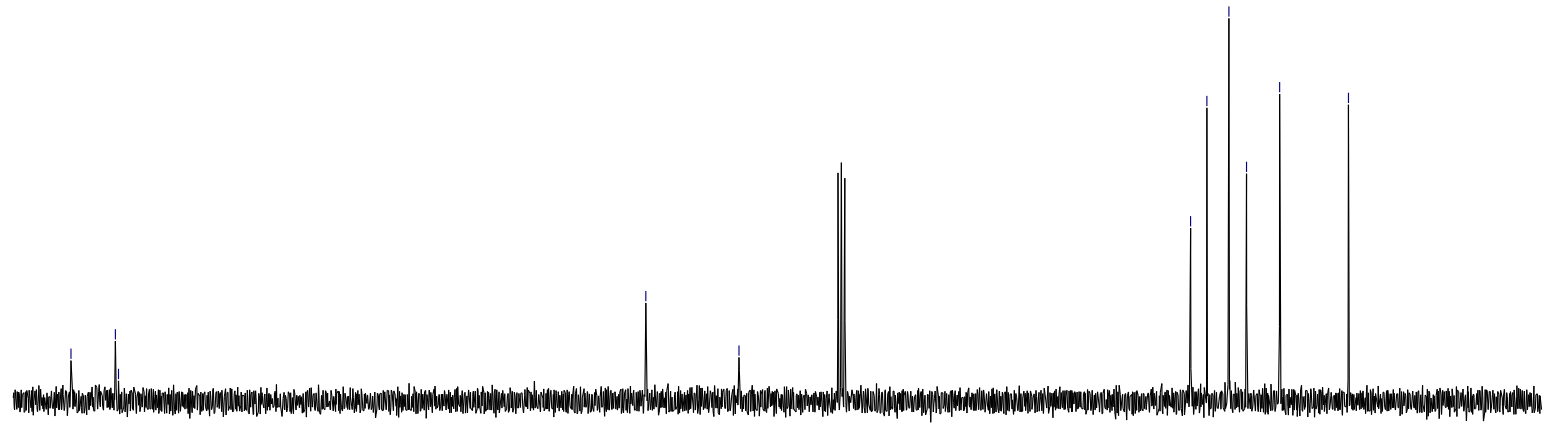

$\begin{array}{llllllllllllllllll}180 & 170 & 160 & 150 & 140 & 130 & 120 & 110 & 100 & \underset{\mathrm{f} 1(\mathrm{ppm})}{80} & 70 & 60 & 50 & 40 & 30 & 20 & 10 & 0\end{array}$

Figure S22: ${ }^{1} H$ NMR (top) and ${ }^{13} C$ NMR (bottom) spectra for compound 19. 


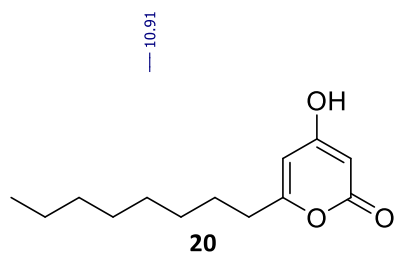

Y่ำ

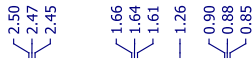

${ }^{1} \mathrm{H} \mathrm{NMR}\left(300 \mathrm{MHz}, \mathrm{CDCl}_{3}\right)$
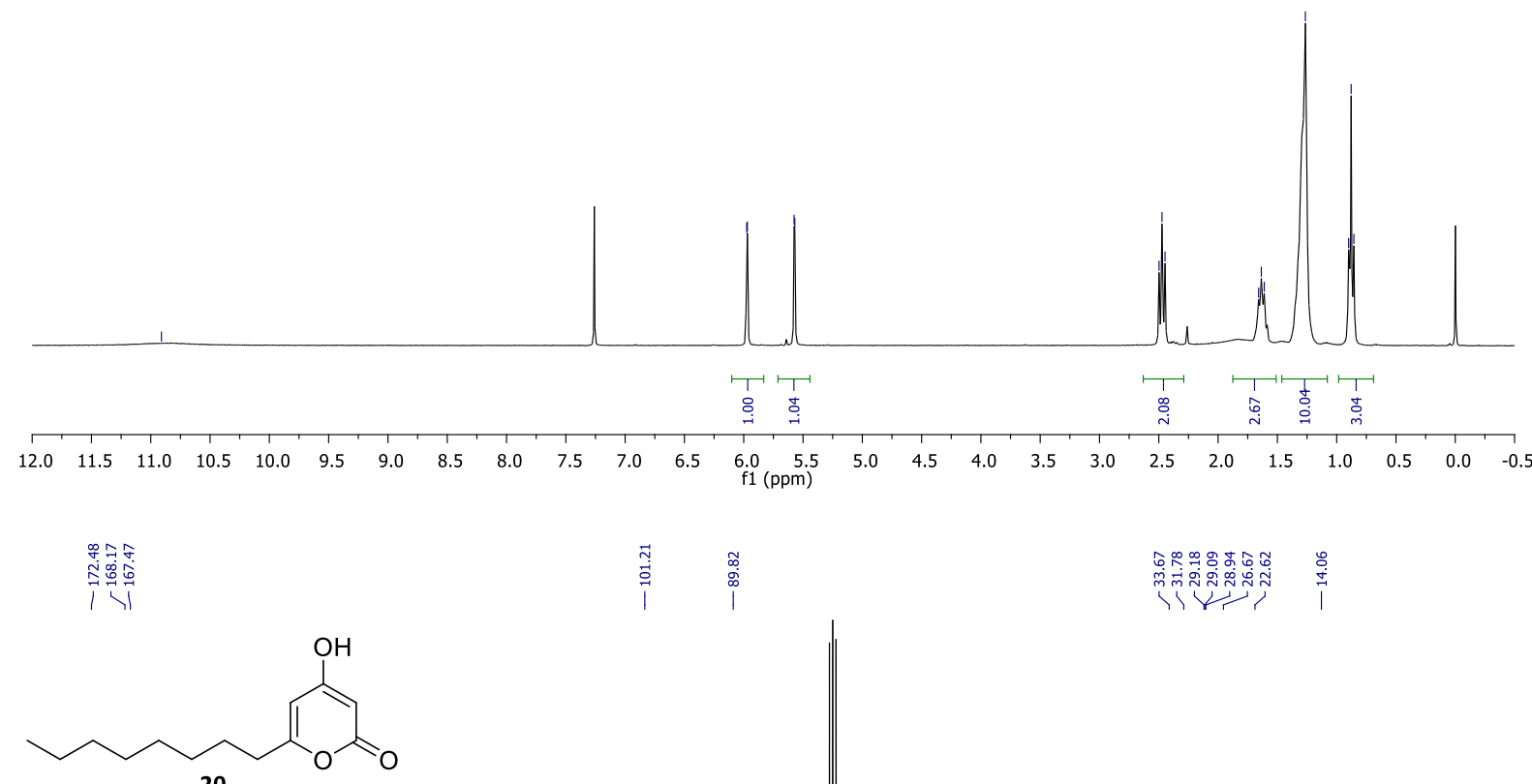

20

${ }^{13} \mathrm{C} \mathrm{NMR}\left(75 \mathrm{MHz}, \mathrm{CDCl}_{3}\right)$

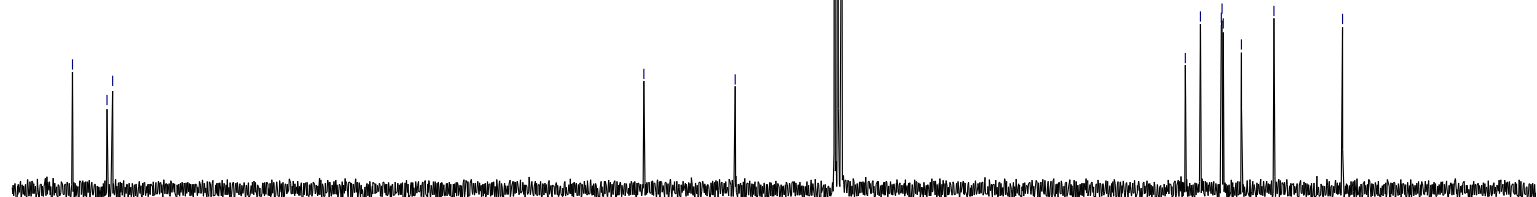

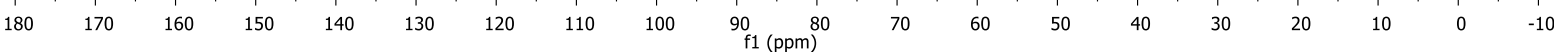

Figure S23: ${ }^{1} \mathrm{H} N \mathrm{NR}$ (top) and ${ }^{13} \mathrm{C} N M R$ (bottom) spectra for compound 20. 


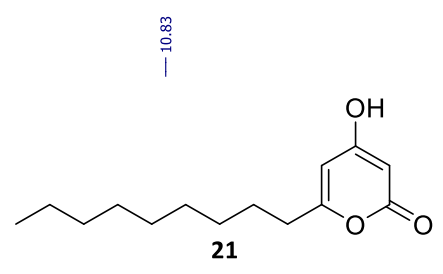

ทे

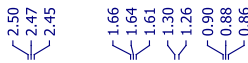

${ }^{1} \mathrm{H}$ NMR $\left(300 \mathrm{MHz}, \mathrm{CDCl}_{3}\right)$
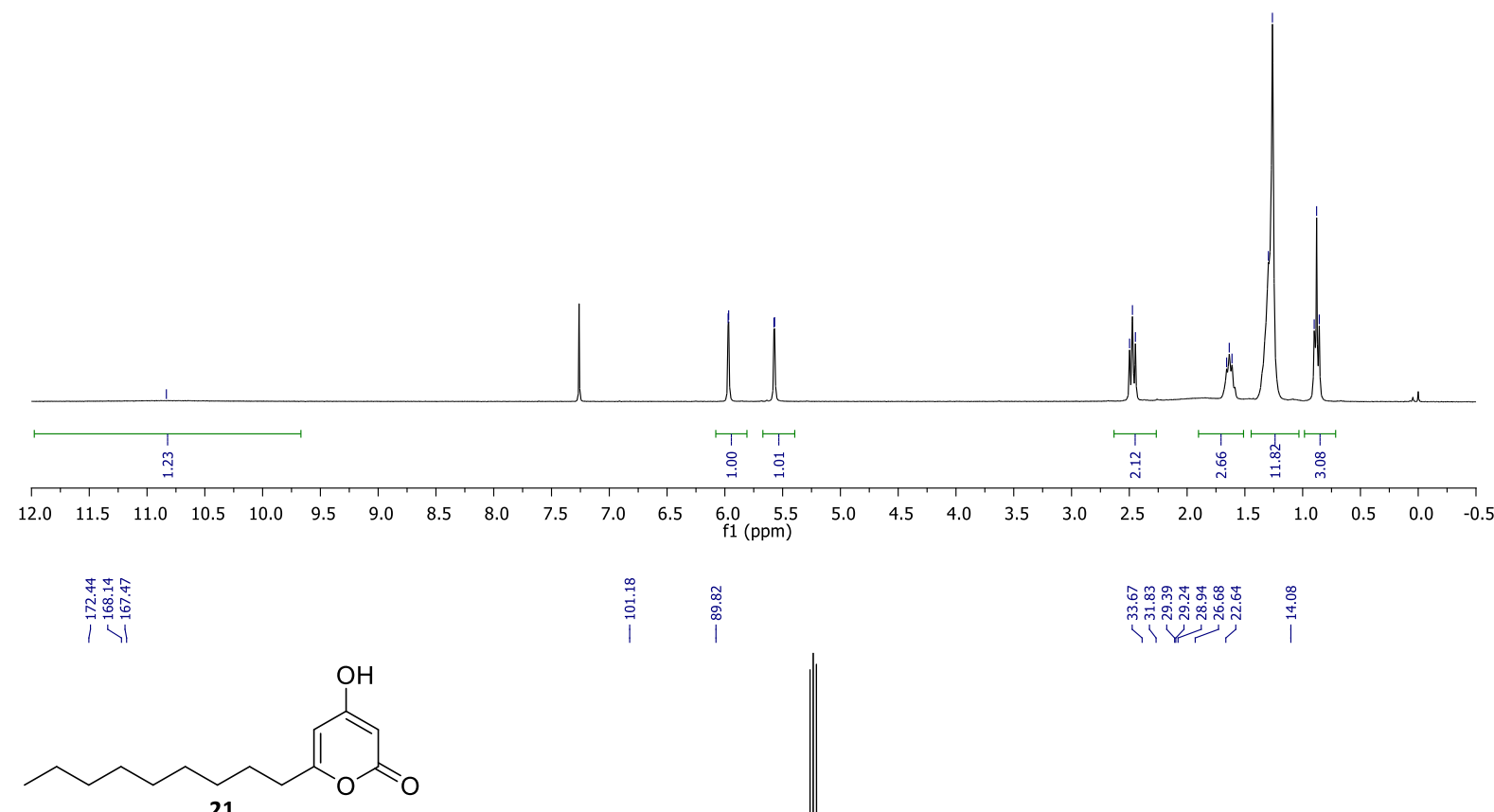

21

${ }^{13} \mathrm{C}$ NMR (75 MHz, $\mathrm{CDCl}_{3}$ )
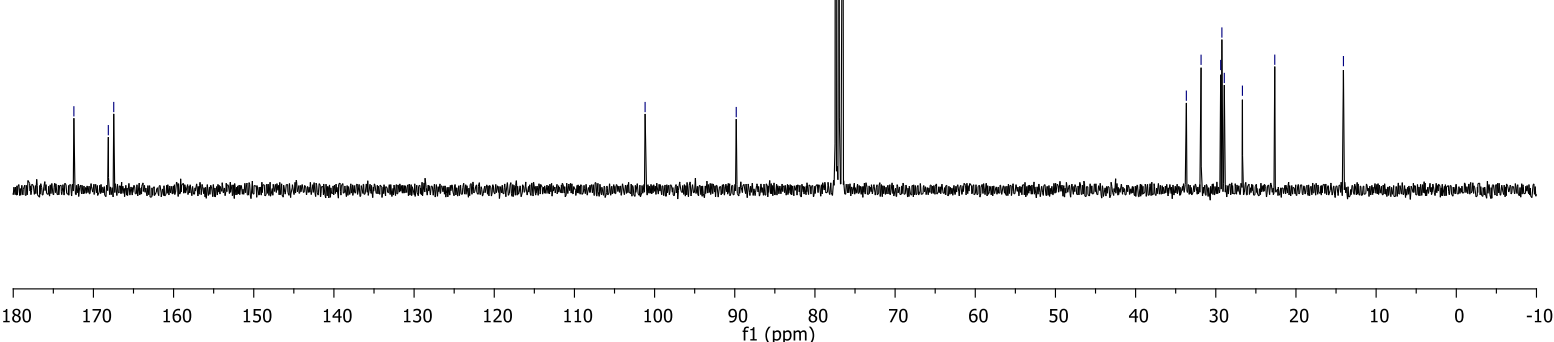

Figure S24: ${ }^{1} \mathrm{H}$ NMR (top) and ${ }^{13} \mathrm{C}$ NMR (bottom) spectra for compound 21. 

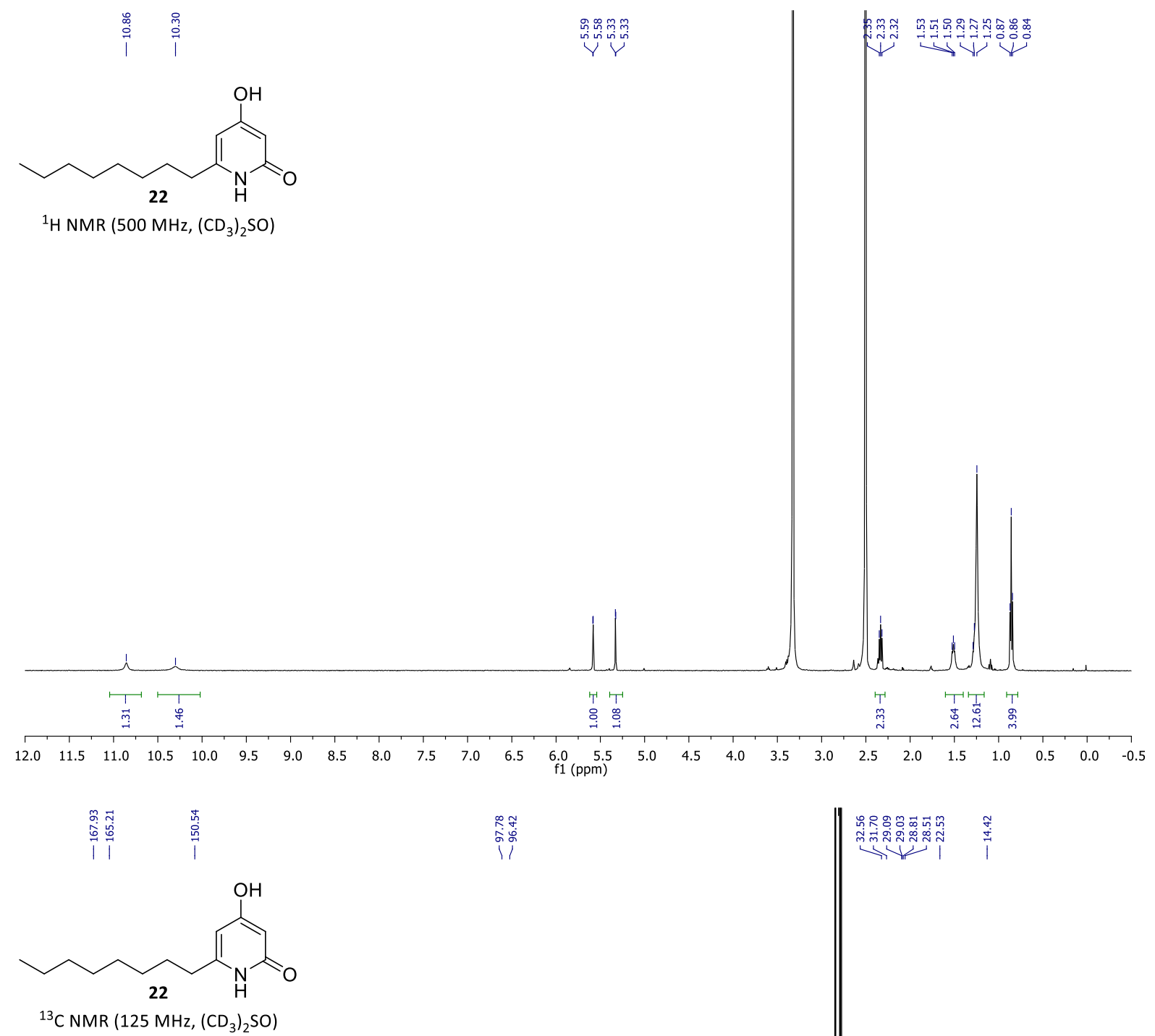

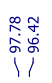

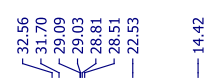
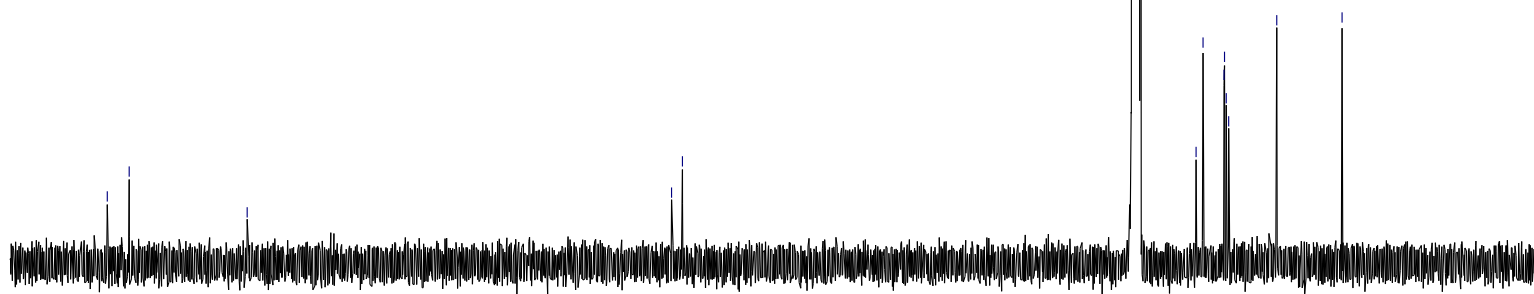

$\begin{array}{llllllllll}180 & 170 & 160 & 150 & 140 & 130 & 120 & 110 & 100 & 90 \\ \mathrm{f} 1(\mathrm{ppm})\end{array}$

Figure S25: ${ }^{1} H$ NMR (top) and ${ }^{13} \mathrm{C}$ NMR (bottom) spectra for compound 22. 


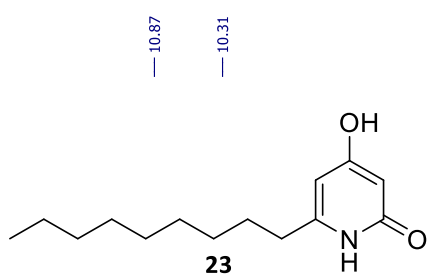

${ }^{1} \mathrm{H}$ NMR $\left(500 \mathrm{MHz},\left(\mathrm{CD}_{3}\right)_{2} \mathrm{SO}\right)$
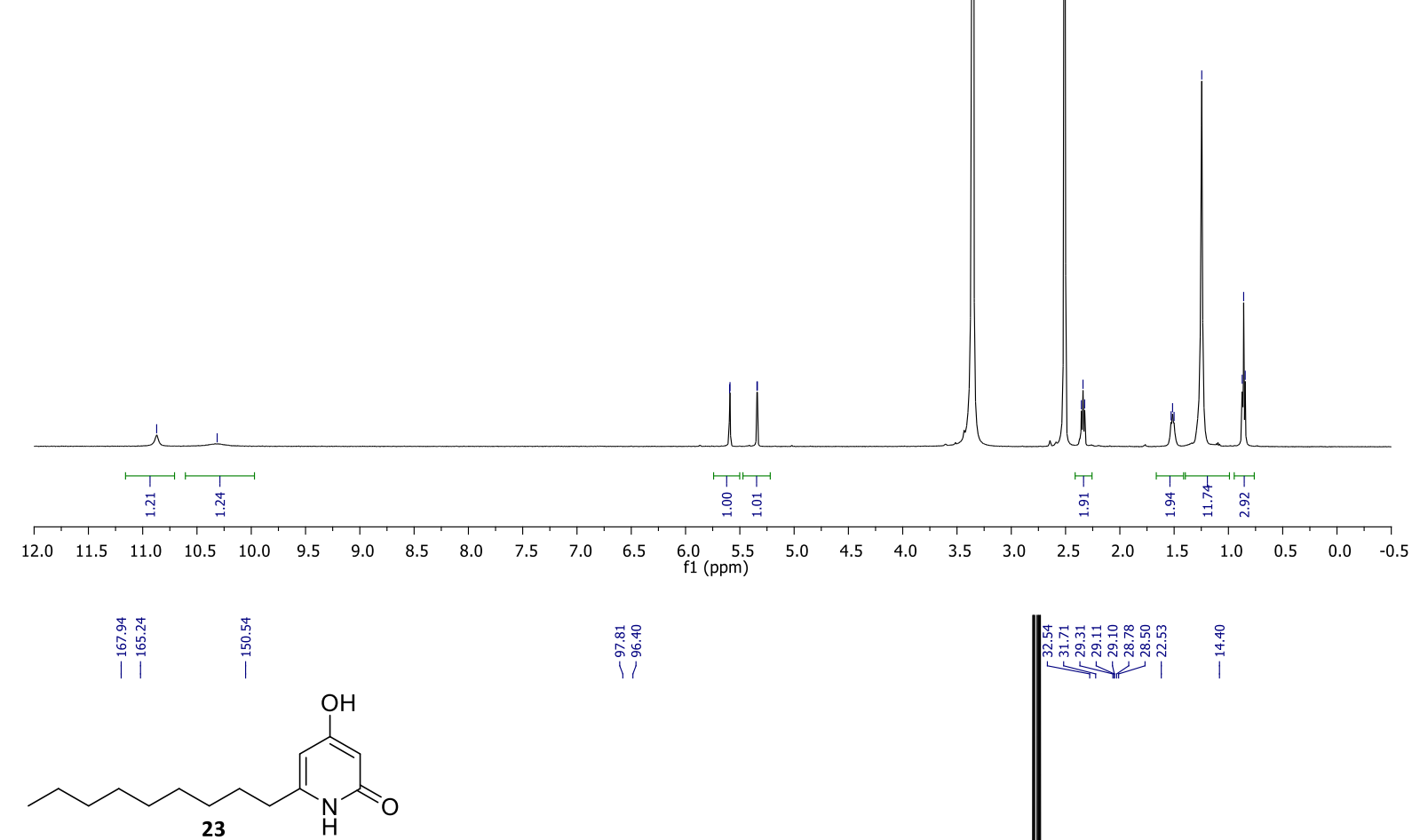

${ }^{13} \mathrm{C}$ NMR (125 MHz, $\left.\left(\mathrm{CD}_{3}\right)_{2} \mathrm{SO}\right)$

|
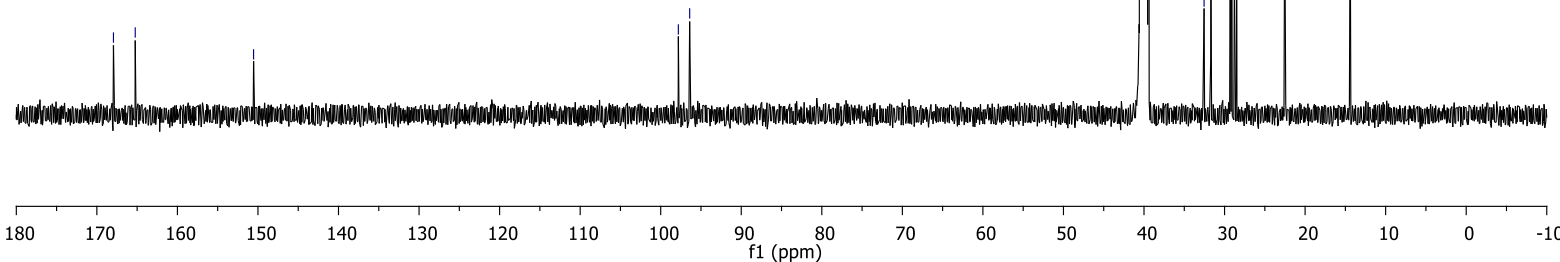

Figure S26: ${ }^{1} \mathrm{H} N \mathrm{NR}$ (top) and ${ }^{13} \mathrm{C}$ NMR (bottom) spectra for compound 23. 


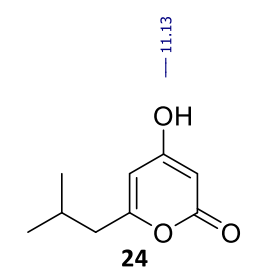

${ }^{1} \mathrm{H}$ NMR $\left(300 \mathrm{MHz}, \mathrm{CDCl}_{3}\right)$

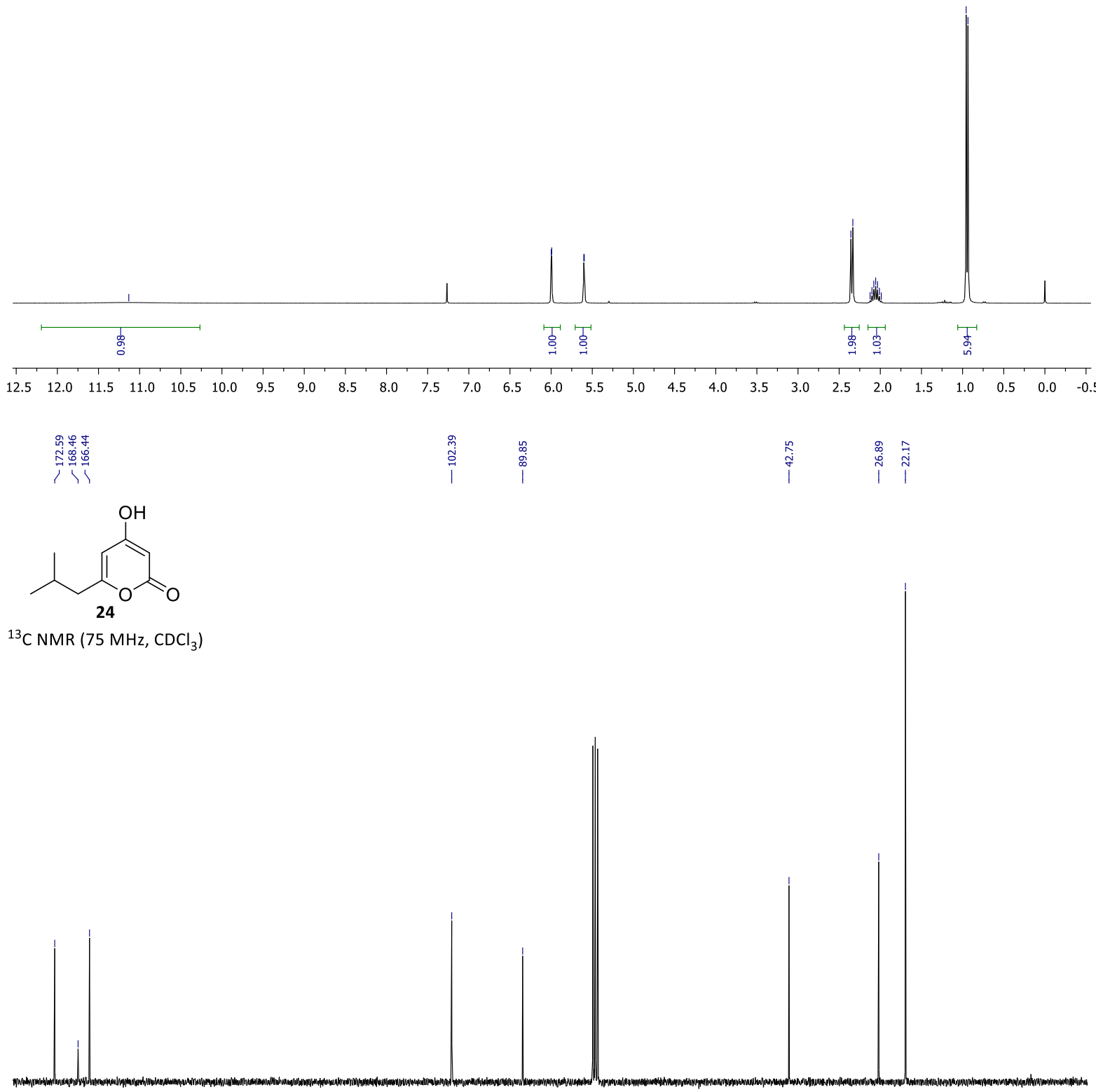

Figure 27: ${ }^{1} \mathrm{H} N \mathrm{NR}$ (top) and ${ }^{13} \mathrm{C} N \mathrm{NM}$ (bottom) spectra for compound 24. 

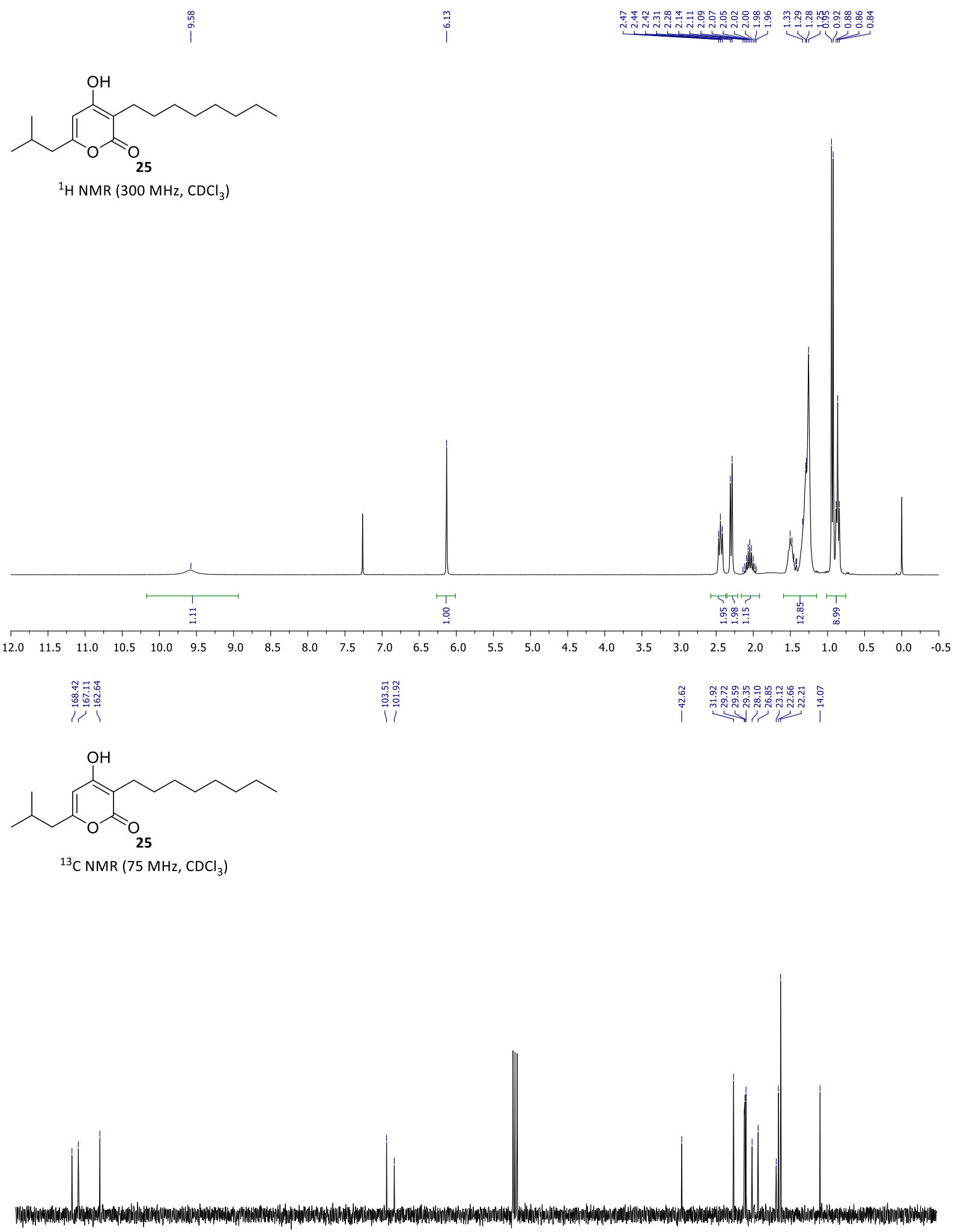

$\begin{array}{lllllllllllllllllllllll}180 & 170 & 160 & 150 & 140 & 130 & 120 & 110 & 100 & 90 & 80 & 70 & 60 & 50 & 40 & 30 & 20 & 10 & 0 & -10\end{array}$

Figure S28: ${ }^{1} \mathrm{H} N \mathrm{NR}$ (top) and ${ }^{13} \mathrm{C} N \mathrm{NM}$ (bottom) spectra for compound 25. 
<smiles>CCCCCCCCCc1c(O)cc(CC(C)C)oc1=O</smiles>

${ }^{1} \mathrm{H}$ NMR (300 MHz, $\mathrm{CDCl}_{3}$ )
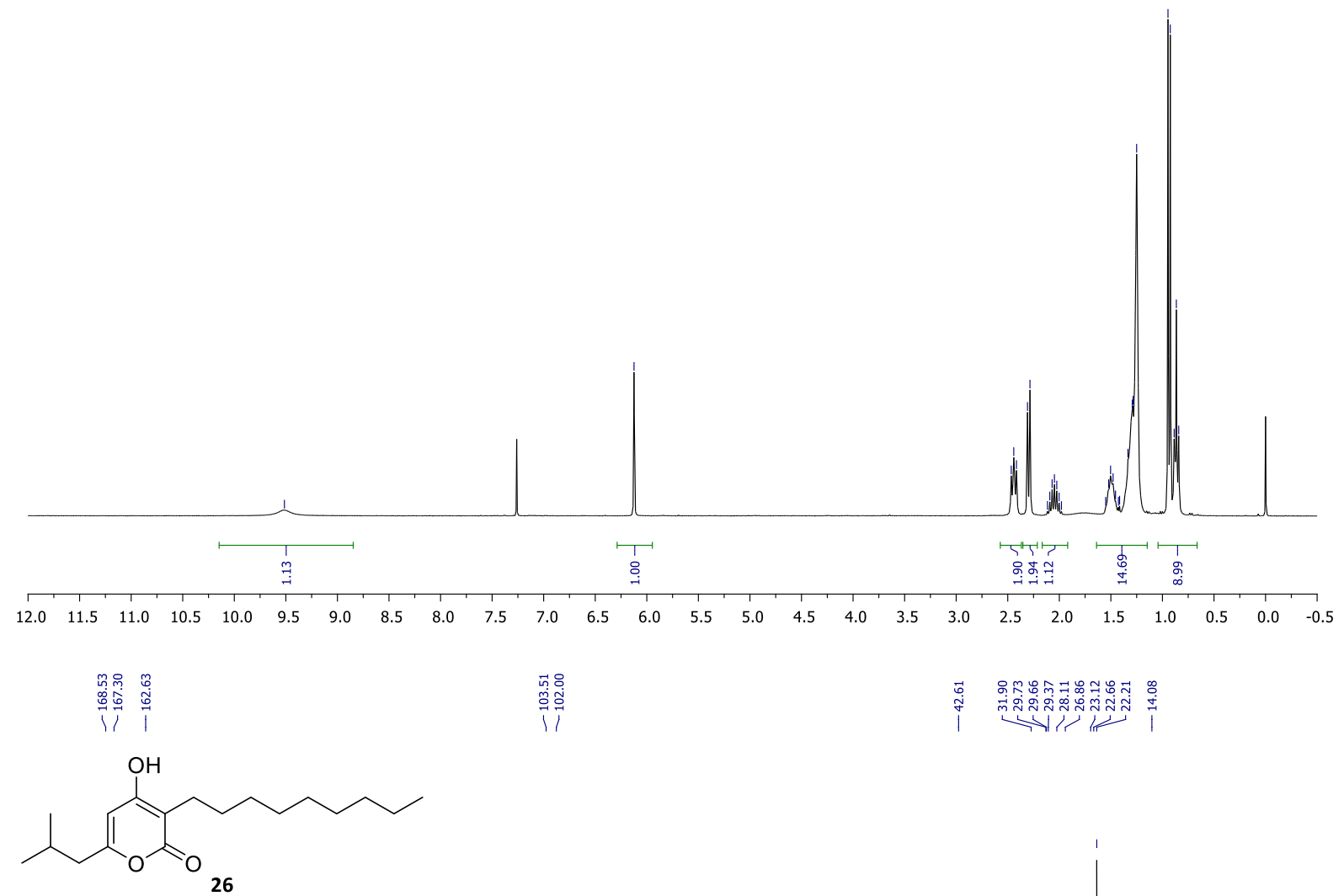

藏通

苞

${ }^{13} \mathrm{C} \mathrm{NMR} \mathrm{(75} \mathrm{MHz,} \mathrm{CDCl}_{3}$ )
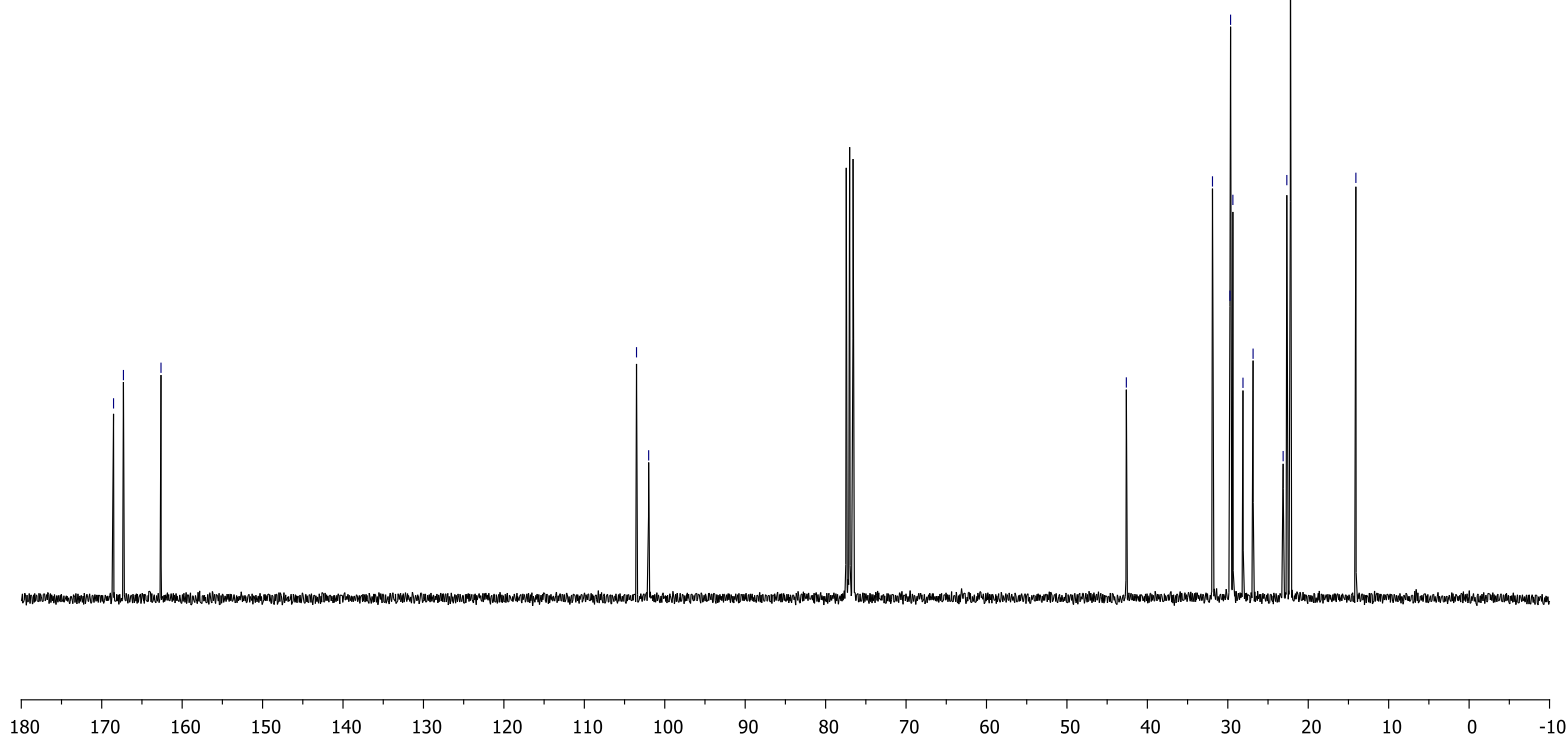

Figure S29: ${ }^{1} \mathrm{H} N \mathrm{NR}$ (top) and ${ }^{13} \mathrm{C}$ NMR (bottom) spectra for compound 26. 

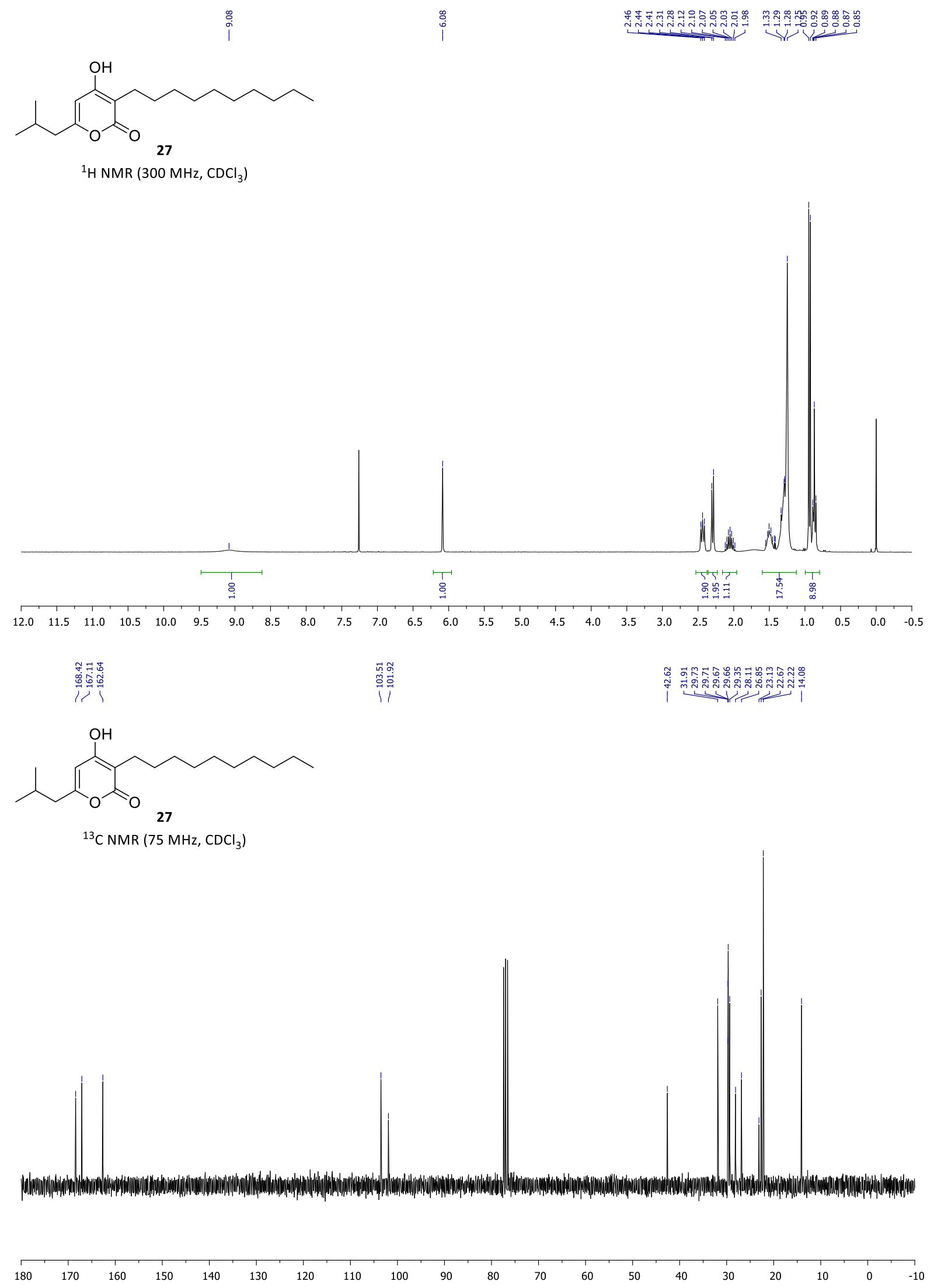

Figure S30: ${ }^{1} \mathrm{H}$ NMR (top) and ${ }^{13} \mathrm{C}$ NMR (bottom) spectra for compound 27. 


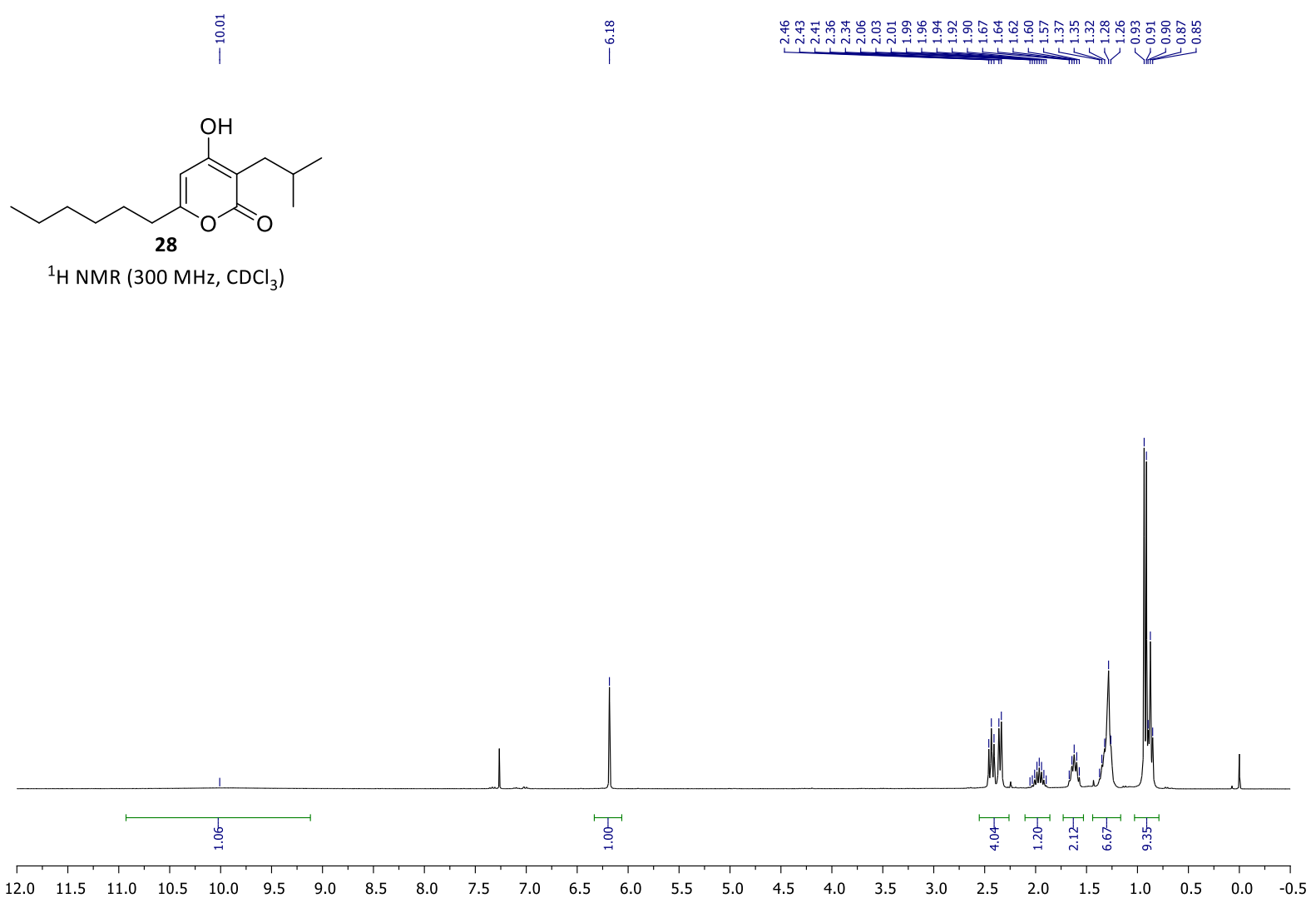

|r|

il

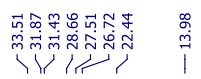

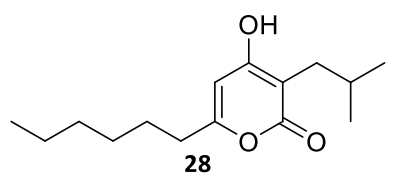

${ }^{13} \mathrm{CNMR}\left(75 \mathrm{MHz}, \mathrm{CDCl}_{3}\right)$

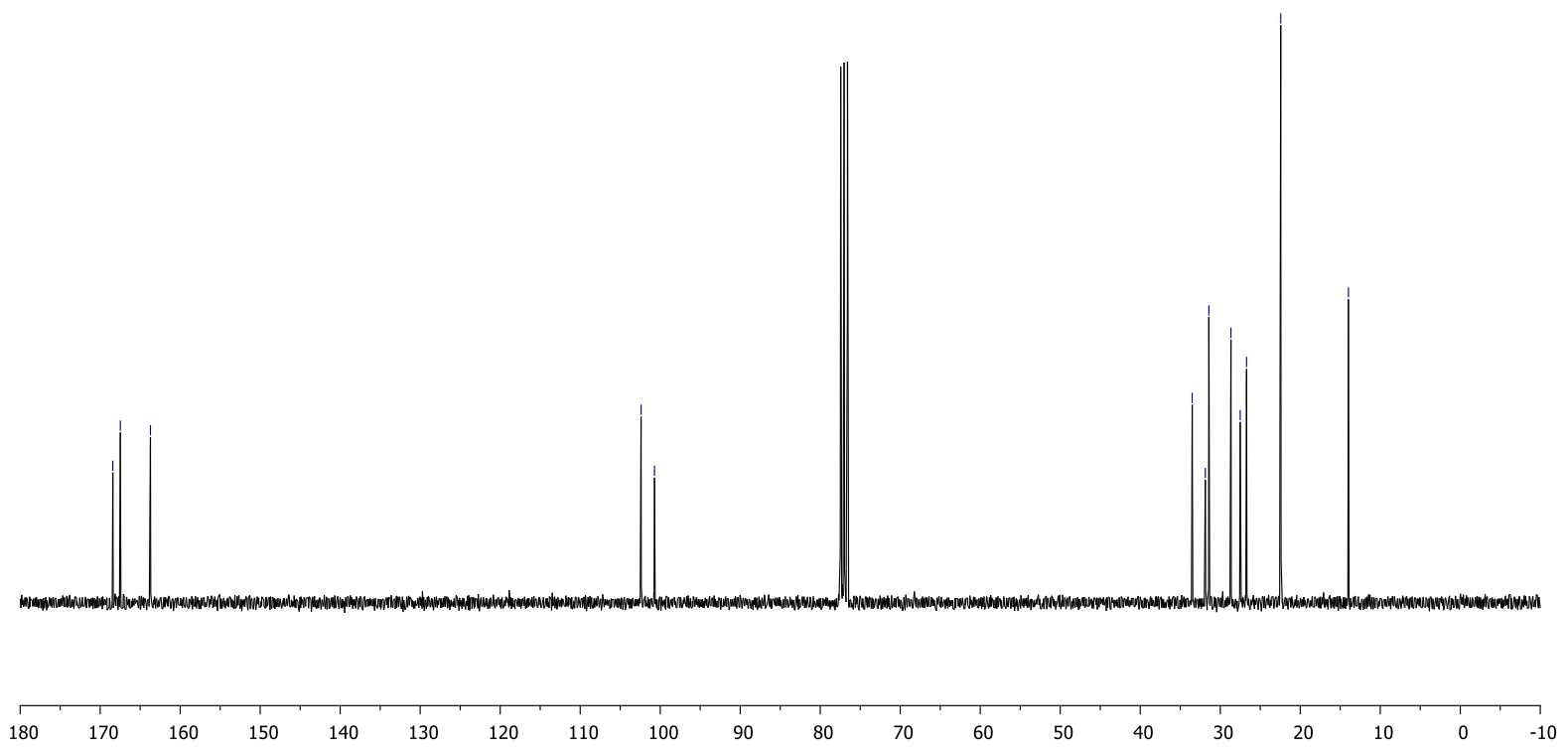

Figure S31: ${ }^{1} \mathrm{H} N \mathrm{NMR}$ (top) and ${ }^{13} \mathrm{C} N \mathrm{NMR}$ (bottom) spectra for compound 28. 


\section{References}

1. Kraus, G. A.; Basemann, K.; Guney, T., Selective pyrone functionalization: reductive alkylation of triacetic acid lactone. Tetrahedron Lett. 2015, 56, 3494-3496.

2. Moreno-Mañas, M.; Pleixats, R., A Method for the Alkylation at C-3 of 4-Hydroxy-6-methyl-2pyrone (Triacetic Acid Lactone). Synthesis 1984, 430-431.

3. Tempone, A. G.; Ferreira, D. D.; Lima, M. L.; Costa Silva, T. A.; Borborema, S. E. T.; Reimão, J. Q.; Galuppo, M. K.; Guerra, J. M.; Russell, A. J.; Wynne, G. M.; Lai, R. Y. L.; Cadelis, M. M.; Copp, B. R., Efficacy of a series of alpha-pyrone derivatives against Leishmania (L.) infantum and Trypanosoma cruzi. Eur. J. Med. Chem. 2017, 139, 947-960.

4. Moreno-Manas, M.; Ribas, J.; Virgili, A., Palladium-catalyzed C-alkylations of the highly acidic and enolic triacetic acid lactone. Mechanism and stereochemistry. J. Org. Chem. 1988, 53, 5328-5335.

5. $\quad$ Swidorski, J. J.; Wang, J.; Hsung, R. P., A Concise Total Synthesis of (-)-Cylindricine C through a Stereoselective Intramolecular Aza-[3 + 3] Annulation Strategy. Org. Lett. 2006, 8, 777-780.

6. Lokot, I. P.; Pashkovsky, F. S.; Lakhvich, F. A., A new approach to the synthesis of 3,6- and 5,6-dialkyl derivatives of 4-hydroxy-2-pyrone. Synthesis of rac-germicidin. Tetrahedron 1999, 55, 4783-4792.

7. Poulton, G. A.; Cyr, T. D., Pyrones. IX. Synthetic approaches to the fungal metabolite phacidin and its derivatives. Can. J. Chem. 1982, 60, 2821-2829.

8. Katritzky, A. R.; Wang, Z.; Wang, M.; Hall, C. D.; Suzuki, K., Facile Syntheses of 2,2-Dimethyl6-(2-oxoalkyl)-1,3-dioxin-4-ones and the Corresponding 6-Substituted 4-Hydroxy-2-pyrones. J. Org. Chem. 2005, 70, 4854-4856.

9. Giddens, A. C.; Nielsen, L.; Boshoff, H. I.; Tasdemir, D.; Perozzo, R.; Kaiser, M.; Wang, F.; Sacchettini, J. C.; Copp, B. R., Natural product inhibitors of fatty acid biosynthesis: synthesis of the marine microbial metabolites pseudopyronines $A$ and $B$ and evaluation of their anti-infective activities. Tetrahedron 2008, 64, 1242-1249.

10. Pardo, L. M.; Prendergast, A. M.; Nolan, M.-T.; Ó Muimhneacháin, E.; McGlacken, G. P., $\mathrm{Pd} /$ Pivalic Acid Mediated Direct Arylation of 2-Pyrones and Related Heterocycles. Eur. J. Org. Chem. 2015, 3540-3550.

11. Demuner, A. J.; Valente, V. M. M.; Barbosa, L. C. A.; Rathi, A. H.; Donohoe, T. J.; Thompson, A. L., Synthesis and phytotoxic activity of new pyridones derived from 4-hydroxy-6-methylpyridin2(1H)-one. Molecules 2009, 14, 4973-4986.

12. Liu, Y.; Zhang, Q.; Chen, L.-H.; Yang, H.; Lu, W.; Xie, X.; Nan, F.-J., Design and Synthesis of 2-Alkylpyrimidine-4,6-diol and 6-Alkylpyridine-2,4-diol as Potent GPR84 Agonists. ACS Med. Chem. Lett. 2016, 7, 579-583. 